\title{
Investigation on the Core Bypass Flow in a Very High Temperature Reactor
}

\section{Reactor Concepts RD\&D \\ Dr. Yassin Hassan \\ Texas A\&M University}

Madeline Feltus, Federal POC

Richard Schultz, Technical POC

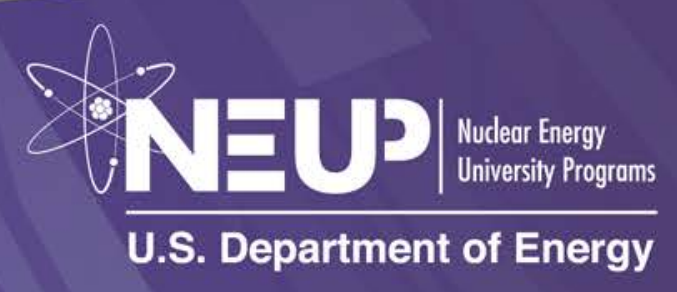




\section{Final Report}

Project Title: Investigation on the Core Bypass Flow in a Very High

Temperature Reactor

Covering Period:

October, 2009-September, 2013

Date of Report:

Friday, October 18, 2013

Recipient:

Name: Texas A\&M University

Street: 3133 TAMU

City: College Station

State: Texas

Zip: 77843

Contact Number: $\quad 90995$

Project Number: $\quad 09-840$

Principal Investigator: $\quad$ Yassin Hassan - 9798457090 - y-hassan@tamu.edu

Project Objective: Investigation of the possible scenarios present in a prismatic modular reactor (PMR) regarding the bypass flow problem is proposed. This work will make use of non-intrusive optical measurement techniques to obtain the dynamic behavior of the complex flow present in the gaps formed by adjacent prisms in various directions. Measurement of full field time-resolved velocity fields will be obtained in the gaps originating the bypass flow and in some of the coolant channels. The amount of coolant flow passing through the gaps between adjacent prims 
will be quantified. Computational fluid dynamics simulation will be performed.

\section{TPOCs:}

Richard Schultz phone: 208-526-9508

Email: Richard.Schultz@inl.gov

Federal POC:

Madeline Feltus phone: 301-903-2308

Email: madeline.feltus@nuclear.energy.gov 


\begin{abstract}
Very High Temperature Rector (VHTR) had been designated as one of those promising reactors for the Next Generation (IV) Nuclear Plant (NGNP). For a prismatic core VHTR, one of the most crucial design considerations is the bypass flow and crossflow effect. The bypass flow occurs when the coolant flow into gaps between fuel blocks. These gaps are formed as a result of carbon expansion and shrinkage induced by radiations and manufacturing and installation errors. Hot spots may appear in the core if the large portion of the coolant flows into bypass gaps instead of coolant channels in which the cooling efficiency is much higher.

A preliminary three dimensional steady-state CFD analysis was performed with commercial code STARCCM+ 6.04 to investigate the bypass flow and crossflow phenomenon in the prismatic VHTR core. The k- $\varepsilon$ turbulence model was selected because of its robustness and low computational cost with respect to a decent accuracy for varied flow patterns. The wall treatment used in the present work is two-layer all y+ wall treatment to blend the wall laws to estimate the shear stress. Uniform mass flow rate was chose as the inlet condition and the outlet condition was zero gauge pressure outlet.
\end{abstract}

Grid independence study was performed and the results indicated that the discrepancy of the solution due to the mesh density was within $2 \%$ of the bypass flow fraction. The computational results showed that the bypass flow fraction was around $12 \%$. Furthermore, the presence of the crossflow gap resulted in a up to $28 \%$ reduction 
of the coolant in the bypass flow gap while mass flow rate of coolant in coolant channels increased by around 5\%. The pressure drop at the inlet due to the sudden contraction in area could be around $1 \mathrm{kpa}$ while the value was about $180 \mathrm{~Pa}$ around the crossflow gap region. The error analysis was also performed to evaluate the accumulated errors from the process of discretization and iteration. It was found that the total error was around $4 \%$ and the variation for the bypass flow fraction was within $1 \%$. 


\section{ACKNOWLEDGEMENTS}

The authors acknowledge the funding from the Nuclear Energy University Program (NEUP) and the US Department of Energy (DOE).

\section{Students Involved:}

1. Huhu Wang - Ph.D. Nuclear Engineering (in progress)

2. Elvis Dominguez-Ontiveros - Post-doctoral research associate Mechanical Engineering (in progress)

3. Worasit Kanjanakijkasem - Ph.D. Mechanical Engineering (2012) 


\section{LIST OF PUBBLICATIONS AND ORAL PRESENTATIONS}

1. Huhu Wang, Elvis Dominguez-Ontiveros, Yassin A. Hassan, "CFD Analysis of Core Bypass Flow and Crossflow in a Prismatic Very High Temperature Gas-cooled Nuclear Reactor Based on a Two-Layer Block Model”, Nuclear Engineering and Design , 2013 (Under revision)

2. Huhu Wang, Elvis Dominguez-Ontiveros, Yassin A. Hassan, "Study of the Core Bypass Phenomena in a Prismatic VHTR”, NURETH 15,, May, 2013, Pisa, Italy

3.Huhu Wang, Elvis Dominguez-Ontiveros, Yassin A. Hassan, "Preliminary CFD Studies of Bypass Flow and Crossflow in VHTR" , American Nuclear Society Winter Meeting, San Diego, CA, November, 2012 (Presentation)

4. Worasit Kanjanakijkasem, Elvis Dominguez-Ontiveros, Yassin A. Hassan, "Preliminary Study of Bypass Flow in Prismatic Core of VHTR", Proceedings of 19th International Conference on Nuclear Engineering (ICONE19), May 16-19, 2011, Chiba, Japan

5. Worasit Kanjanakijkasem, Elvis Dominguez-Ontiveros, Yassin A. Hassan, "Design of New Facility for Bypass Flow Experiment", the 25th Conference of the Mechanical Engineering Network of Thailand, October 19 - 21, 2011, Krabi, Thailand 


\section{NOMENCLATURE}

\begin{tabular}{|c|c|}
\hline BG & Bypass Flow Gap \\
\hline CG & Crossflow Gap \\
\hline ID & Internal Diameter \\
\hline $\mathrm{Re}$ & Reynolds Number \\
\hline $\operatorname{Re}_{\mathrm{CH}}$ & Reynolds Number based on the Coolant Channels \\
\hline$\rho$ & Fluid Density \\
\hline $\mathrm{D}$ & Hydraulic Diameter \\
\hline $\bar{u}$ & Average Velocity \\
\hline$\mu$ & Fluid Dynamic Viscosity \\
\hline $\mathrm{P}$ & Pressure \\
\hline $\mathrm{V}$ & Velocity \\
\hline$\phi$ & Flow Variable (velocity or pressure) \\
\hline $\mathrm{F}$ & Strength of the Convection of Flow \\
\hline$\Omega_{i j}$ & Vorticity Tensor \\
\hline$u_{*}$ & Friction Velocity \\
\hline$\tau_{w}$ & Wall Shear Stress \\
\hline$\delta$ & Boundary Thickness \\
\hline $\mathrm{L}$ & Turbulence Length Scale \\
\hline$\Delta V_{i}$ & Volume of $i_{\text {th }}$ Domain \\
\hline $\mathrm{N}$ & Total Number of Cells \\
\hline
\end{tabular}

viii 


$\begin{array}{ll}\mathrm{h} & \text { The Representative Mesh Size } \\ \mathrm{v}_{\mathrm{b}} & \text { Average Velocity in the Bypass Flow Gap } \\ R_{e B G} & \text { Reynolds Number based on the Bypass Flow Gap } \\ \xi & \text { Pressure Loss Factor } \\ \Delta \mathrm{P} & \text { Pressure Drop } \\ L_{t u r b u l e n t} & \text { Entrance Length for the Turbulent Flow } \\ \delta w & \text { Accumulated Error } \\ \delta u_{i} & \text { Independent Random Errors } \\ \varepsilon_{\mathrm{d}} & \text { Discretization Error } \\ \varepsilon_{\mathrm{I}} & \text { Iterative Convergence Error }\end{array}$




\section{TABLE OF CONTENTS}

PART I: NUMERICAL ANALYSIS

Page

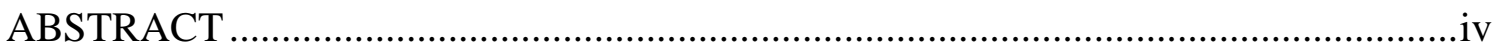

ACKNOWLEDGEMENTS …………………....................................................

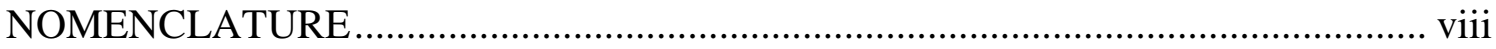

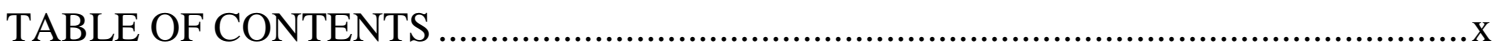

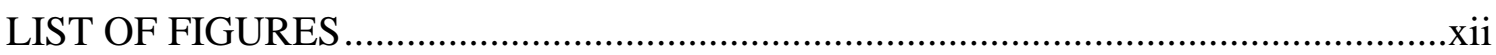

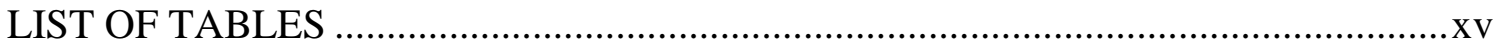

1. INTRODUCTION

1.1 Very High Temperature Gas-cooled Reactor (VHTR) ………………............ 1

1.2 Bypass Flow and Crossflow Phenomenon in a VHTR Core ……………......... 4

1.3 Previous Works Review ...........................................................................

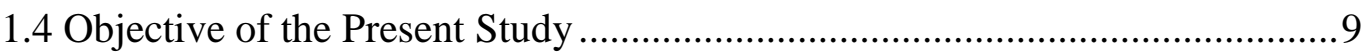

2. REFERENCE EXPERIMENTAL FACILITY ………...........................................

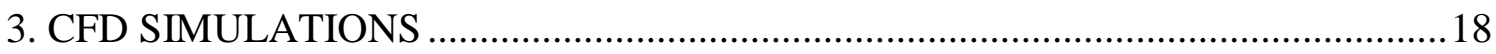

3.1 Computational Domain ............................................................................ 18

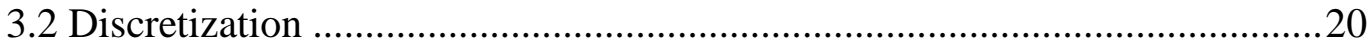

3.3 Physics Models and Boundary Conditions.....................................................24

3.3.1 Differencing Scheme ...................................................................24

3.3.2 Turbulence Modeling .................................................................2

3.3.3 Near Wall Treatment ....................................................................29

Page 
3.3.4 Setup for Other Models .................................................................... 33

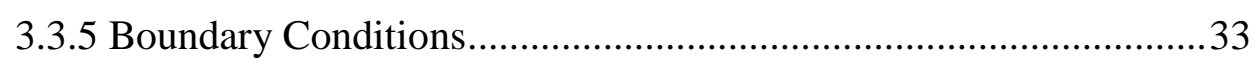

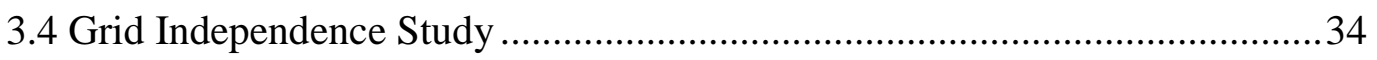

3.4.1 Discretization Errors vs. Grid Resolution ..........................................34

3.4.2 Richardson Extrapolation Approach .....................................................

3.4.3 Results of the Grid Independence Study ……………………….......38

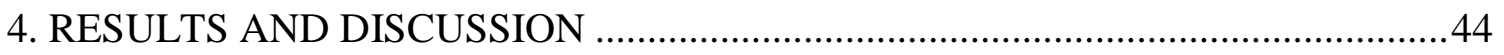

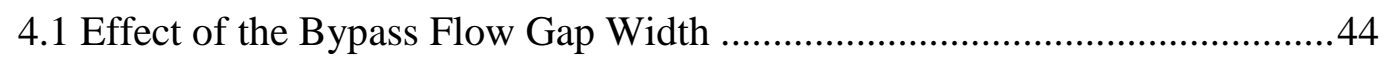

4.2 Effect of the Crossflow Gap.........................................................................

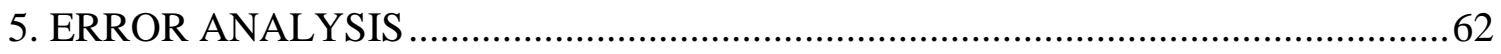

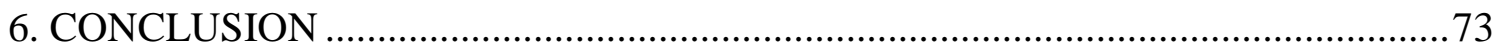

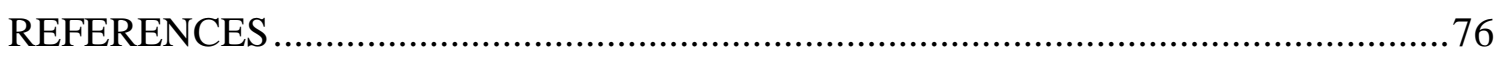

\section{PART II: EXPERIMENTAL STUDIES}

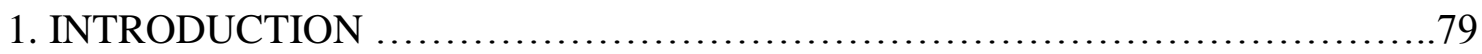

2. EXPERIMENTAL SETUP …........................................... 79

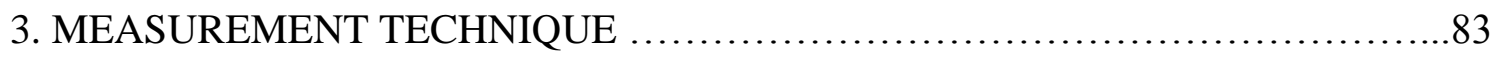

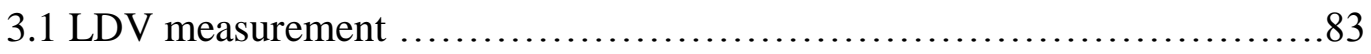

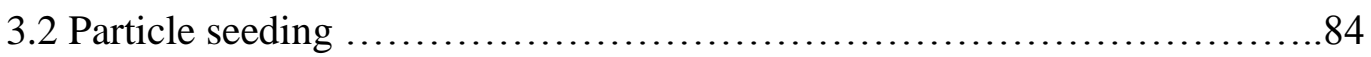

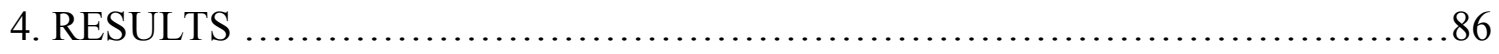

5. UNCERTAINTY ANALYSIS _........................................... 97

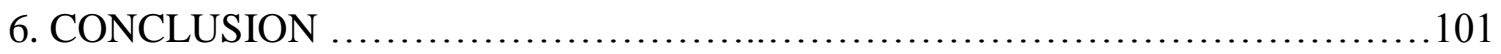




\section{LIST OF FIGURES}

FIGURE $\quad$ Page

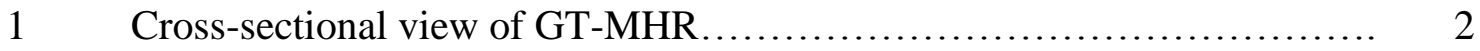

2 Core Arrangement of GT- MHR ..................................................... 3

3 The formation process of the VHTR fuel............................................ 4

4 Graphite Dimensional Change as a Function of Irradiation Fluence ............ 5

5 Sketch of the bypass flow and crossflow in a prismatic VTHR core....... 6

6 Scaling process from GA Concept ${ }^{*}$ (left) to the TAMU Model (right) ........ 13

$7 \quad$ TAMU Experimental Facility ........................................................ 14

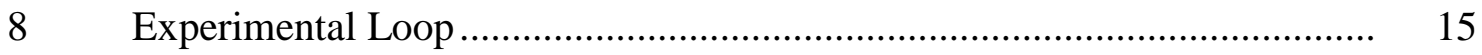

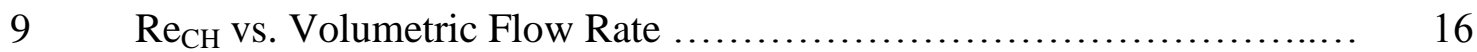

10 Computational Domain ............................................................. 19

11 Illustration of the Multi-Region Meshing Technique …........................... 23

12 Illustration of Conformal Mesh on the Interface of the Stagnation Box and the Bypass Gap ................................................................................ 24

13 Control Volume for the One-dimension Diffusion-Convection Problem..... 25

14 Second-Order Upwind Differencing Scheme..................................... 26

15 Subdivisions of the Near-Wall Region for the Turbulent Flow ................... 30

16 Two Approaches for the Wall Treatment .......................... 31

17 Discretization Error vs. Number of Cells ............................................ 35

18 Bypass Flow Fraction vs. Volumetric Flow Rate (BG6CG1)..................... 39

xii 
19 Bypass Flow Fraction vs. Volumetric Flow Rate (BG4CG1).................... 40

20 Bypass Flow Fraction vs. Volumetric Flow Rate (BG2CG1)................... 40

21 Bypass Flow Fraction vs. Volumetric Flow Rate (All Cases) .................... 42

22 Bypass Flow Fraction vs. $\operatorname{Re}_{\mathrm{BG}}$ for Varied Bypass Flow Gap Widths.......... 45

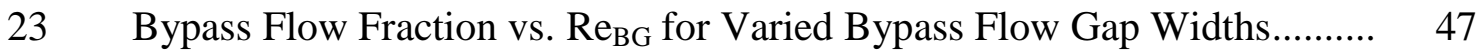

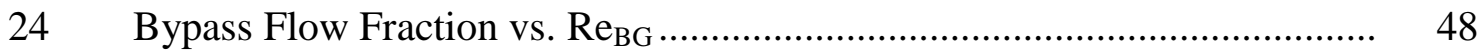

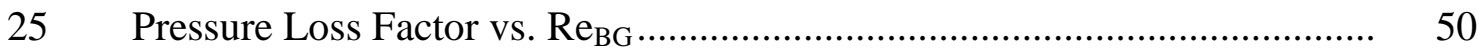

$26 \quad$ Velocity Field for the Uniform Gap Case ........................... 51

27 Velocity Field for the Wedge-Shape Gap Case ..................................... 52

28 Sketch of Planes Used for Calculation ................................................ 53

29 Mass Flow Rate on Plane A for the Uniform Gap Case ............................ 54

30 Mass Flow Rate Change for the Uniform Gap Case ............................... 55

31 Mass Flow Rate Change for the Wedge-Shape Gap Case ......................... 56

32 Pressure Distribution within the Crossflow Gap .................................... 57

33 Static Pressure Distribution ........................................................... 58

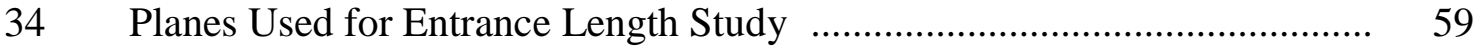

35 Velocity Profile in a Coolant ............................................................ 60

36 Velocity Profile in coolant channel \#A and \#F ...................................... 61

37 Residuals for the BG4CG0 case with a flow rate of $80 \mathrm{gpm}$...................... 65

38 Mass Flow Rate of the Bypass Flow at the Outlet as a function of the number of iterations........................................... 65

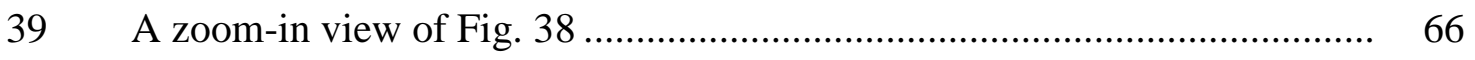


40 Grid Independence Study Results with Error Bars

41 Flow Chart of the Deterministic Method to Calculate the Accumulated Errors. 70

42 Bypass Flow Fraction as a Function of $\mathrm{Re}_{\mathrm{BG}}$ with Accumulated Errors....... 71

$43 \quad$ Experimental loop................................................. 80

44 Improvements of the upper plenum and the supporting frame................81

45 TSI LDV system borrowed for test purpose.............................82

46 Flow conditioner in previous design (left) and new design...................83

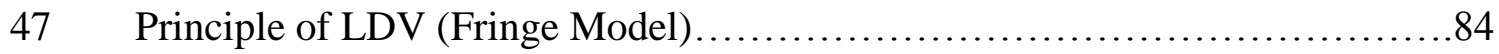

48 Particle injecting methods which did not work due to the backpressure:

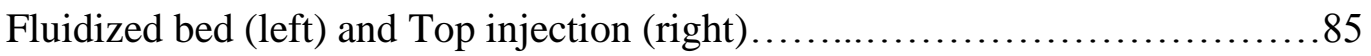

49 Size distribution of the chalk power (left) and the Fog machine (right)

None of these two is good for LDV measurements ........................86

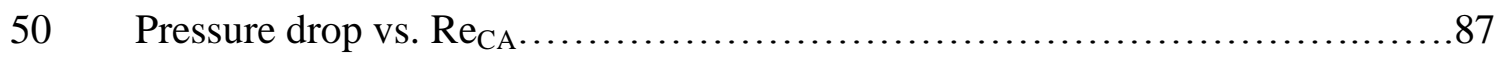

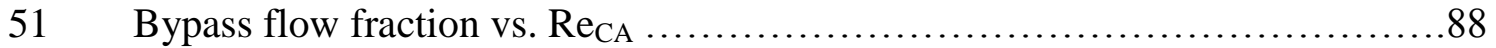

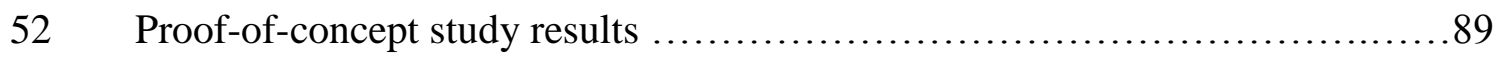

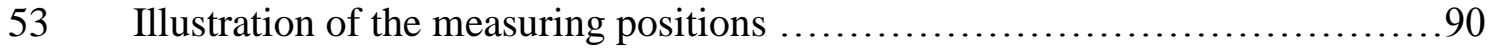

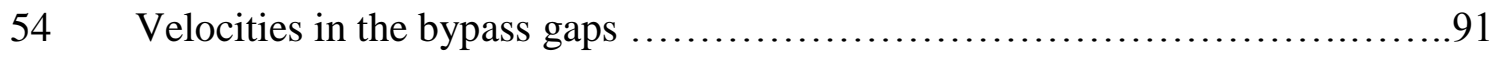

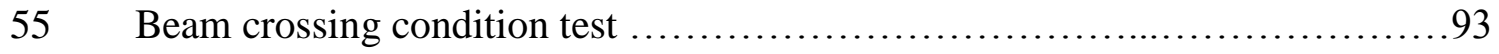

56 Examination of the optics alignment ...................................94

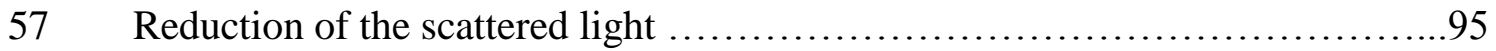

58 Signals obtained at $\mathrm{y}=20 \mathrm{~mm}$ (left) and $\mathrm{y}=110 \mathrm{~mm}$ (right) at $\mathrm{P} 2$ position ....96

59 Fig.59 Histogram obtained at $\mathrm{y}=110 \mathrm{~mm}$ at $\mathrm{P} 1$ position with $\mathrm{Re}_{\mathrm{BG}}=9,990 \ldots 97$ 
60 The calibration Apparatus ............................................ 98

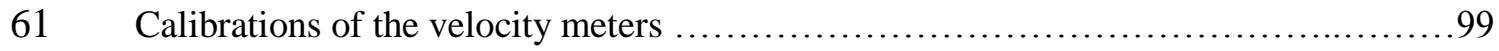

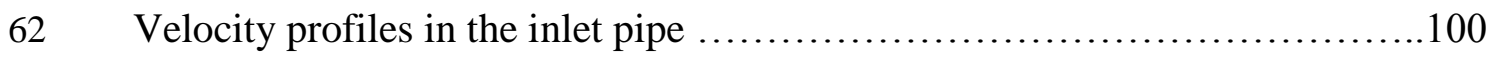

\section{LIST OF TABLES}

TABLE $\quad$ Page

1 Summary of the Dimensions for the GA Concept and the TAMU Model.... 12

2 Summary of Parameters for Mesh Generation (Case BG6CG1) ........... 22

3 Summary of Cases Investigated for the Grid Independence Study............. 38

$4 \quad$ Total Number of Cells for Each Case Investigated ................................ 44

$5 \quad$ Sources of Errors in CFD Modeling ...................................................... 63

$6 \quad$ Variations of the target bypass gap along the $\mathrm{Y}$ direction $\ldots \ldots \ldots \ldots \ldots \ldots \ldots . \ldots 92$

7 Comparisons between the weighted average velocities and the maximum .

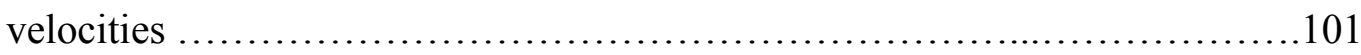




\section{PART I: NUMERICAL ANALYSIS}

\section{INTRODUCTION}

Climate change, air pollution and energy shortage in the past decades had given rise to renewed concerns over the ability of nuclear energy to meet the fast growth of electricity demand. Since Very High Temperature Gas-cooled Reactor (VHTR) was selected as one of the six types of nuclear reactors for Next generation (Generation IV) nuclear plants in 2002, it has been a popular research topic for both National Labs and Universities around the world. This chapter will introduce a basic background of VHTR and the significance of core bypass flow and crossflow in the reactor core. Also, some fundamental knowledge of Computational Fluid Dynamics (CFD) and its applications will be covered. A literature review and purpose of present study close this chapter.

\subsection{Very High Temperature Gas-cooled Reactor (VHTR)}

As early as in 2002, led by US, Generation IV International Forum (GIF) selected six types of nuclear reactors to serve for the Next Generation (IV) Nuclear Plant (NGNP). The Very High Temperature Rector (VHTR) is designated as one of those candidates because of its high efficiency and high outlet temperature $\left(\sim 950^{\circ} \mathrm{C}\right)$ and possible application in the process of hydrogen production as well as coal gasification. This reactor concept is a thermal spectrum nuclear reactor moderated by graphite and cooled by helium. It employs the Brayton cycle to increase the efficiency of reactor. 
VHTR will use tristructural-isotropic (TRISO) fuel for its high integrity and high pressure boundary of keeping fission product gases leaking to the outside of the reactor (J. W. Sterbenz, 2007). The core can be either pebble bed (Chinese HTR-10 or PBMR under development in South Africa) or Prismatic blocks (GT-MHR under development by General Atomics) as shown in Fig. 1.

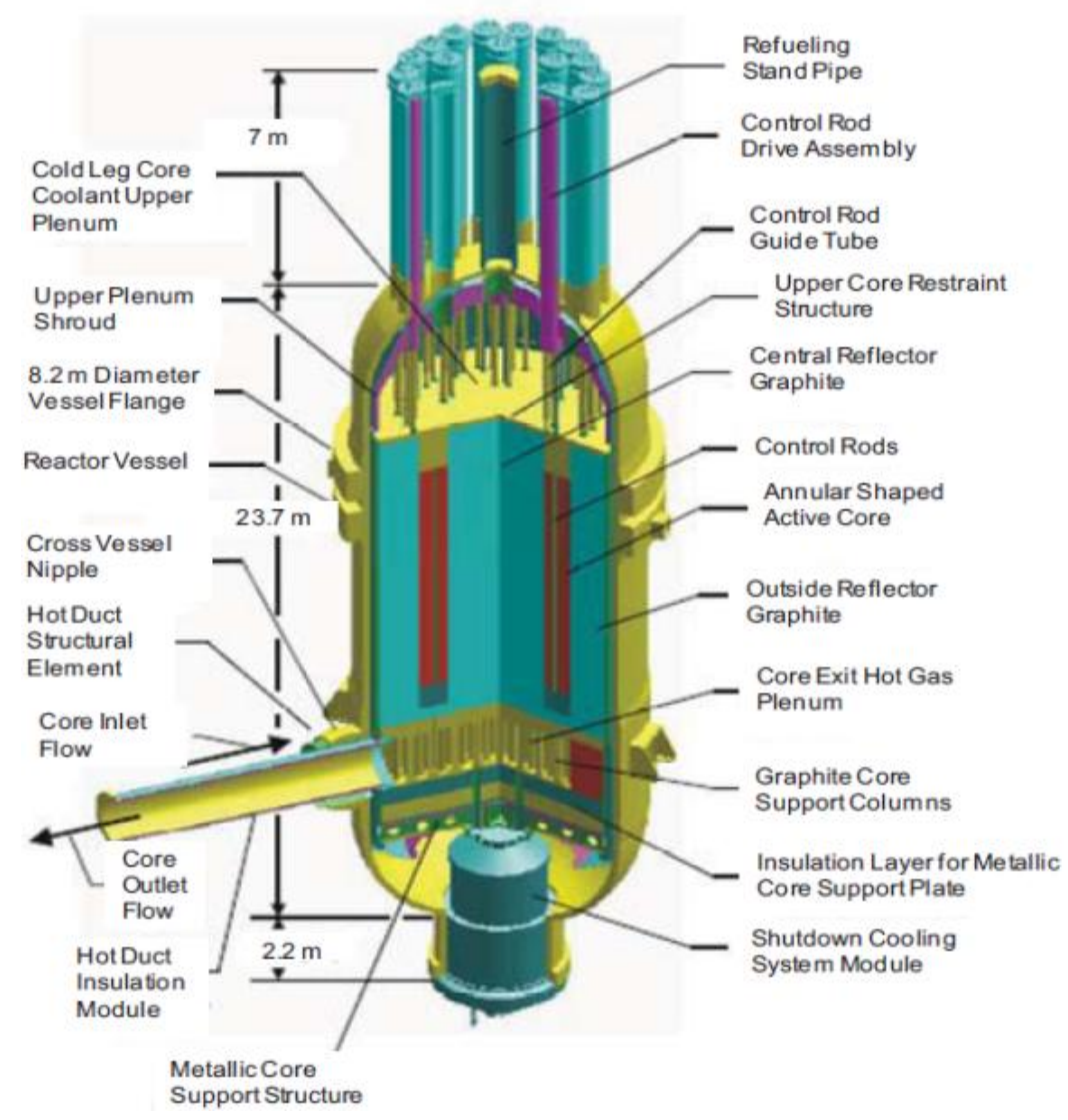

Fig. 1 Cross-sectional view of GT-MHR (P.E., MacDonald, 2003)

It is seen that the prismatic VHTR is mainly composed by annular active core consisting of prismatic fuel blocks, inner and outer graphite reflectors and other 
necessary safety control components. The inherent safety characteristics based on its strong negative fuel temperature coefficient, helium cooled feature and large mass of graphite will enable VTHR to meet the safety requirement of the NGNP.

The core arrangement is presented in Fig. 2 and the red annular part is the fuel region while the remaining white region is the reflector. The fuel assembly consists of fuel holes and coolant channels as shown and the heat is transferred from the hot fuels to the graphite first and then removed by cold helium out of the core. Neutrons are produced in the annular core region and the graphite reflects those leaking neutrons back to the fuel region to initialize more sustainable chain reactions.

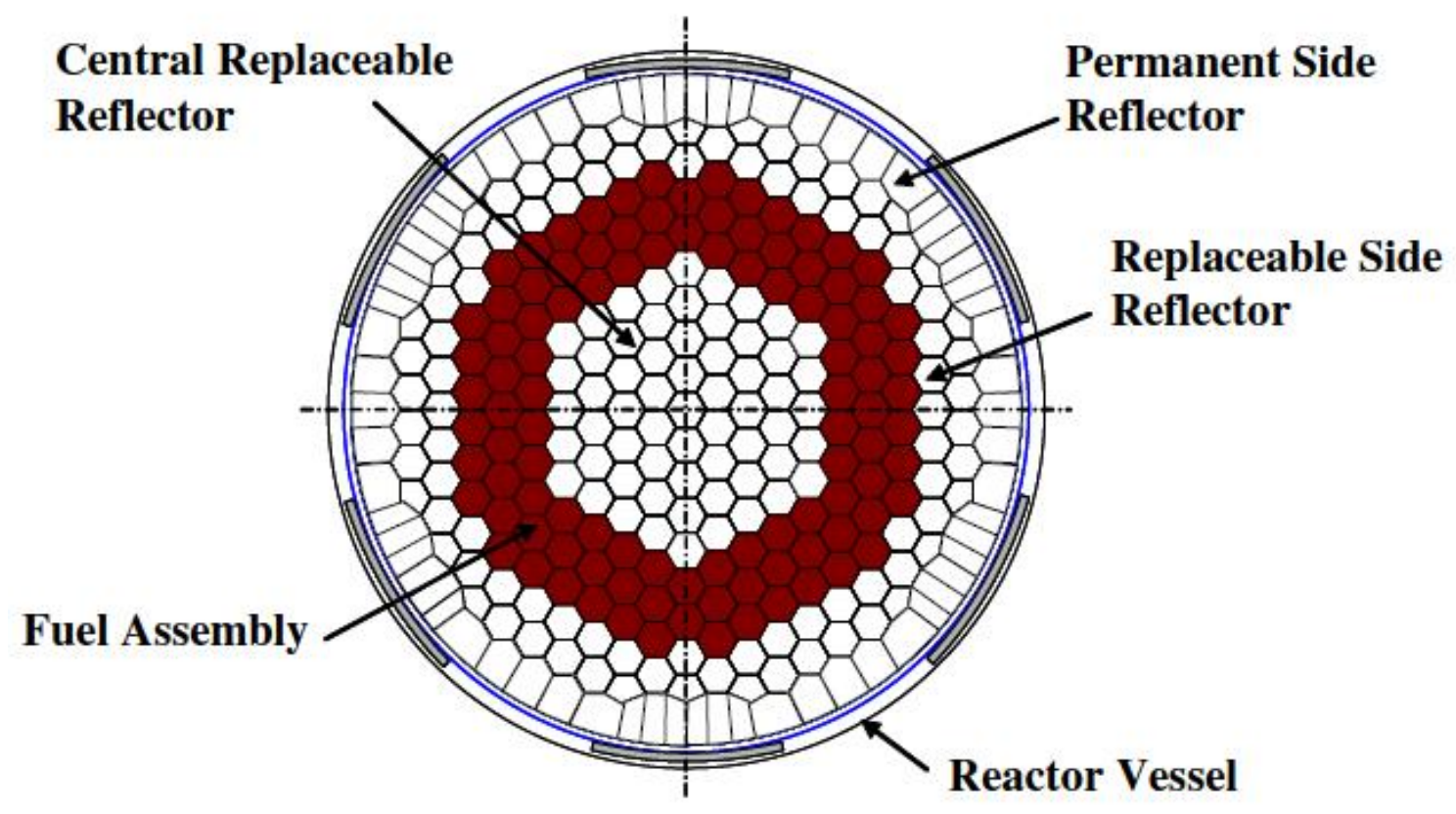

Fig. 2 Core Arrangement of GT- MHR (Tak, N.I., Kim, M.-H., et al., 2008) 
The fuel used for the VHTR is called TRISO fuel which has fuel kernel in the center of the sphere and several layers surrounding the kernel to keep the fission gases inside the fuel and also to increase the structural strength. The TRISO fuel is mixed with graphite to form the fuel compact which will be inserted into the fuel holes to form the fuel block. Fig. 3 presents the formation of the fuel used in the prismatic VHTR.

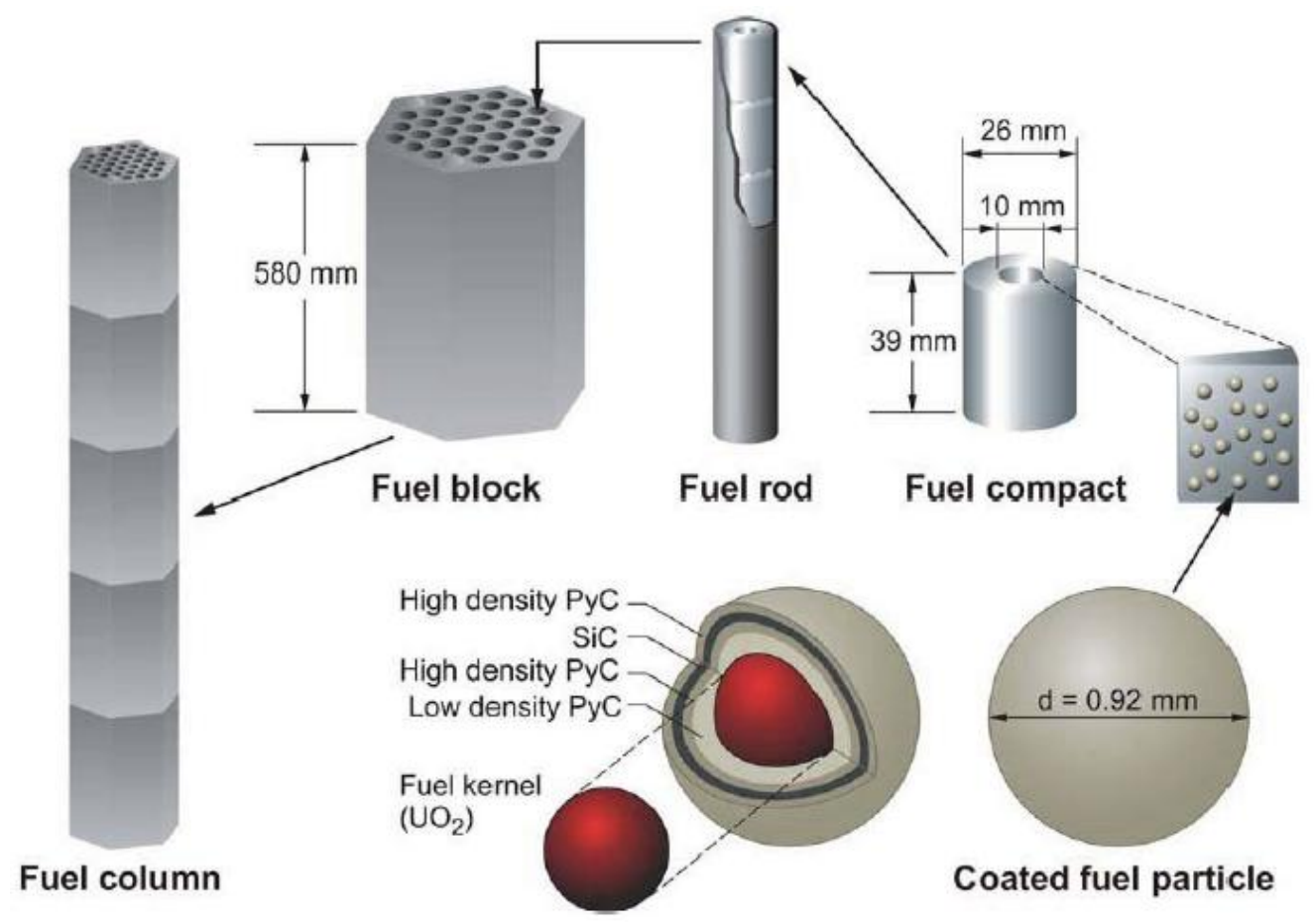

Fig. 3 The formation process of the VHTR fuel (J. Ortensi, J.J., Cogliati, 2010)

\subsection{Bypass Flow and Crossflow Phenomenon in a VHTR Core}

For the prismatic core VHTR, the bypass flow and crossflow effect are important design considerations. The bypass flow occurs when the coolant flows into gaps between fuel blocks. These gaps are formed as a result of carbon dimensional changes including 
expansion and shrinkage induced by radiation exposure, thermal stress, and manufacturing tolerances. Fig. 4 presents the linear change of dimensions as a function of the neutron fluence.

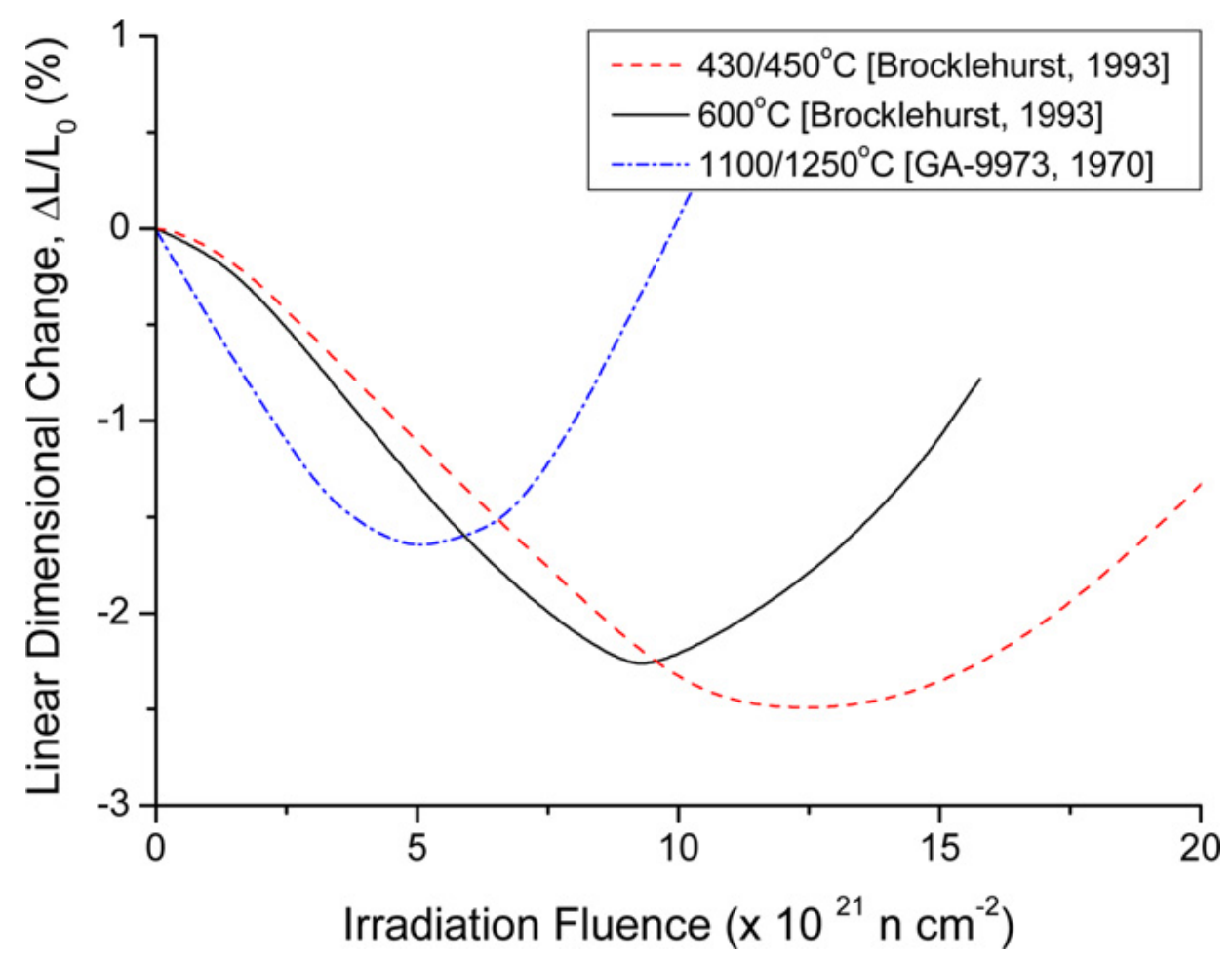

Fig. 4 Graphite dimensional change as a function of irradiation fluence

(Kim, M. H., Lim, H. S. 2011)

The reason why this issue has attracted so much attention is that there would be a risk of fuel melting if the fraction of bypass flow becomes too high as less coolant would flow into coolant channels at which the cooling is more efficient. Also, there is another gap between fuel blocks which are vertically connected called cross gap. These two types of gaps are illustrated in Fig. 5. The bypass flow gap and crossflow gap will result 
in the coolant redistribution in the reactor core. Hot spots will appear if the coolant flow fails to cool down the core evenly and efficiently. In addition, the block will be titled due to the volume change of the graphite, which will increase the uncertainty of the coolant flow distribution in the core area.

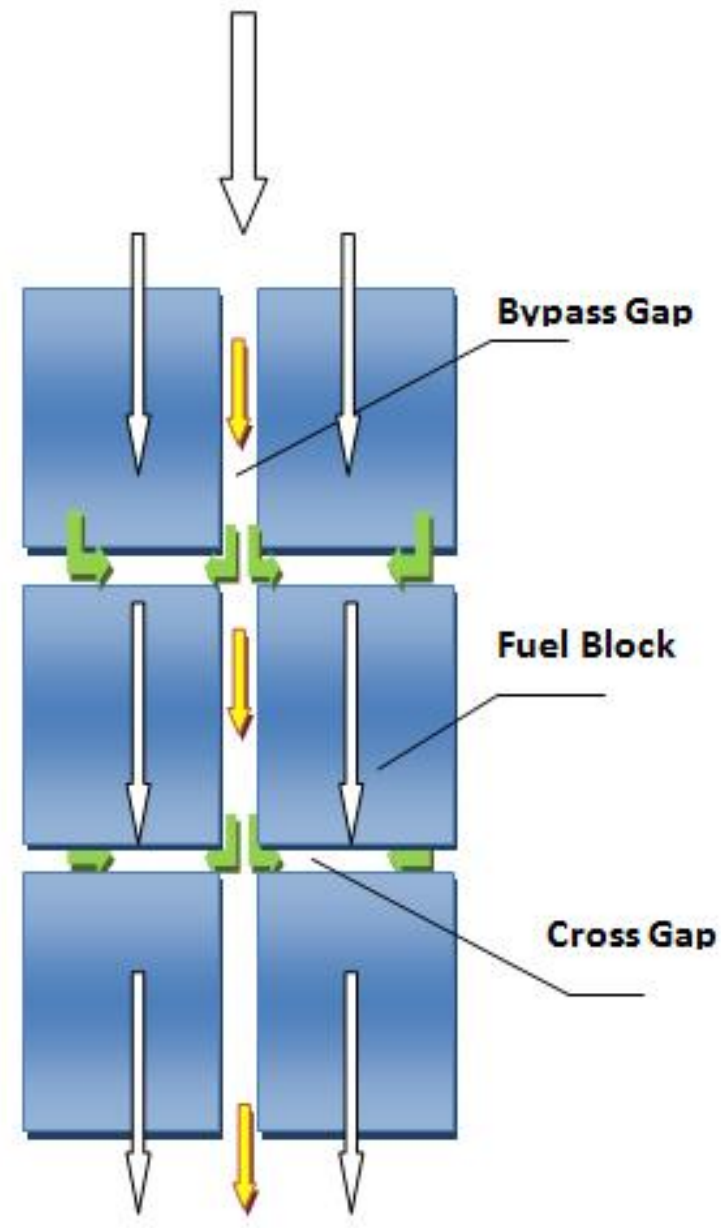

Fig. 5 Sketch of the bypass flow and crossflow in a prismatic VTHR core 


\subsection{Previous Works Review}

In 1977, Fort St. Vrain VHTR in Colorado, US was reported to have experienced 37 fluctuations including inlet temperature of primary coolant and steam generator main temperature in a rise to power test program and finally bypass flows and cross flows were blamed for that abnormal phenomenon (Olson, H. G., Brey, H. L., et al. 1982). A few studies had been conducted focusing on the flow distribution in these gap regions after that scenario. Experiment performed by H.G. Groehn investigated the influence of cross flow on the main flow distribution for a HTGR (H. G. Groehn, 1980) but the result indicated that there were up to $72 \%$ flow reduction because of the cross flow which was not realistic in the practical reactor operating conditions. Basic and full scale experiments carried out in Japan (Kaburaki, H. and Takizuka, T. 1985) found that the leakage flow or cross flow could be described well by the two dimensional laminar flow between parallel planes. Also the authors came up a simple flow model which established a relationship between the mass flow rate and pressure difference to predict the leakage flow in VHTR fuel elements.

Later on, numerical method such as on one dimensional based flow network analysis and finite element method were employed to investigate the effect of the width variation of the bypass and cross flow gap as well as cross flow rates, which agreed well with their experimental data accord to the author (Kaburaki, H. and Takizuka, T. 1987,1990).

In recent several years, with rapid development of computer hardwares and decreasing of the computational cost, computation fluid dynamic (CFD) codes have been 
widely used in the nuclear industry, especially in the field of thermal hydraulic design of the nuclear reactors. A three dimensional simulation using commercial CFD code CFX 11 was performed by N. Tak (Tak, N.I., Kim, M.-H., et al. 2008). A simple unit cell model which was a one-twelfth sector of a typical fuel blocks were applied to evaluate the temperature distribution within the fuel blocks. A similar simulation carried out by H. Sato (Sato, H., Johnson, R., and Schultz, R. 2010) also employed this simplified model to investigate the effect of gap width, turbulence models and uniform and cosine power profiles. Both teams found that bypass flow had a significant influence on the cooling effect of the fuels. And the possibility for the fuels have hot spots is proportional to the width of the bypass flow gap. More parameters were investigated including the total heat generation, graphite block geometry (Johnson, R.W., Sato, H. 2012) and graphite surface roughness (Tung, Y. H., Johnson, R. W., Sato, H. 2011). They concluded that the presence of bypass flow gap flow and increase of the total heat generation as well as the surface roughness increased the maximum outlet coolant temperature and its variation as well. In addition, different turbulence models were compared and the results indicated that the realizable k- $\varepsilon$ model provided an acceptable estimation of the flow characteristics in the bypass flow region. The related experiment which will be used to validate the CFD results is in progress in Idaho National Lab (INL).

Besides in US, several works regarding the bypass flow can crossflow phenomenon in the VHTR core have been performed both experimentally and numerically in Korea. Experiments based on a multi-block air test facility indicated that 
the bypass flow fraction was independent of the inlet mass flow condition and crossflow plays a significant role in the flow and pressure distributions. Also, axial bypass flow gap profile played an important practical factor in determining the bypass flow distribution since the bypass flow gap was not uniform in reality. CFD commercial package CFX-12 was adapted to perform the computational work and the results agreed well with the experimental data (S.J. Yoon, J.H. Lee, et al, 2011, 2012). A procedure to estimate the bypass flow gap size variations during the core lifetime was developed so that the influence from the irradiation, thermal expansions could be examined quantitatively. It was found that the neutron fluence and the temperature distribution in the core had a significant influence on the local sizes of the bypass flow gaps which in turn change the coolant flow distributions through the core (Kim, M. H., Lim, H. S. 2011).

\subsection{Objective of the Present Study}

The intent of present study is to evaluate the capability of commercial CFD code STARCCM+ in investigating the bypass flow and crossflow phenomenon in a prismatic VHTR core. A two-block prismatic VHTR experimental setup built in Texas A\&M University is chosen as the reference facility. The effect of width of bypass flow gap on the coolant flow distribution will be studied the gap sizes vary from $2 \mathrm{~mm}$ to $6 \mathrm{~mm}$. In addition, the influence of the presence of the uniform and wedge-shape crossflow gaps is to be evaluated numerically. The simulation results will provide the experimental researchers a preliminary results regarding with the coolant velocity and pressure 
distribution in the facility so that proper experimental procedures can be designed and also appropriate instruments such us pressure taps can be selected. Furthermore, results from present study will be validated against with the experimental data in future. 


\section{REFERENCE EXPERIMENTAL FACILITY}

The CFD model in present simulation was produced based on the experimental facility built in Texas A\&M University. The experimental apparatus consists of fuel blocks, bypass gap, collector, gap divider and other related supporters and sealing components. It has two layers and each layer includes three one-third sections of one whole block. Considering the objective of the experiment is to investigate bypass flow and crossflow phenomenon adiabatically, the fuel rods are ignored and there are six coolant channels only in each one-third section. The diameter was properly chosen to match the Reynolds number in a real operating condition. The material of the facility is acrylic which has the same refraction index with the P-cymene, the running fluid for the experiment, which enables researchers to employ the Matched Index of Refraction (MIR) method.

The reference experimental facility is designed based on the GA concept. Table 1 compares the dimensions of the GA concept and the TAMU model. The main guideline for the design of the TAMU model is to match the Reynolds number in the practical operation and porosity of the GA concept VHTR. The reason why these two parameters are crucial and so kept the same during the scaling process is that Reynolds number determines the flow characteristics while the porosity influences the relative distribution of the coolant in the coolant channels and in the bypass flow gaps. That is why the coolant diameter of the TAMU model is much larger than that in the GA concept since the number of coolant channels are much smaller in the TAMU model. For the height of 
the core and width across the flats, TAMU model keeps the scale factor constant which is 0.6. Since the widths of the bypass flow gap and the crossflow gap are unknown so far the estimated values are chosen for the TAMU model and the scale factor is 1.0.

Table 1 Summary of the dimensions for the GA concept and the TAMU model

\begin{tabular}{|c|c|c|l|}
\hline Parameter & GA concept & TAMU model & \multicolumn{1}{|c|}{ Scale Factor } \\
\hline BG width & Unknown & $2 \mathrm{~mm}, 4 \mathrm{~mm}, 6 \mathrm{~mm}$ & 1.0 \\
\hline CG width & Unknown & $\begin{array}{c}1 \mathrm{~mm} \text { (Uniform) \& 1- } \\
2 \mathrm{~mm} \text { (Wedge-shape) }\end{array}$ & 1.0 \\
\hline Coolant hole ID & $15.9 \mathrm{~mm}$ & $25.4 \mathrm{~mm}$ & 1.6 \\
\hline Effective core height & $793 \mathrm{~mm}$ & $500 \mathrm{~mm}$ & 0.6 \\
\hline Width across flats & $360 \mathrm{~mm}$ & 233.8 & 0.6 \\
\hline Porosity & 0.2 & 0.2 & 1.0 \\
\hline Re ${ }_{\mathrm{CH}}^{1}$ & $\begin{array}{c}\text { up to } 6 \times 10^{4} \text { at full }^{2} \\
\text { power }\end{array}$ & $1.2 \times 10^{4} \sim 3.8 \times 10^{4}$ & 1.0 \\
\hline
\end{tabular}

${ }^{1} \mathrm{Re}_{\mathrm{CH}}$ is the Reynolds number in the coolant channels;

${ }^{2}$ It is an estimated value based on a report from INL (R.R. Schultz, 2011).

Fig. 6 presents the scaling process from the GA concept to the TAMU model. To keep the bypass gap intact one third of each neighboring block was selected. That is, there are three rhomboids in each layer and total of six rhomboids for the whole facility. The fuel and coolant channels in the GA concept are represented by the enlarged coolant holes in the TAMU model to match the Reynolds number and porosity. The length of the 
side for each rhomboid is $135 \mathrm{~mm}$ and the inner diameter of the coolant holes is 25.4 mm.

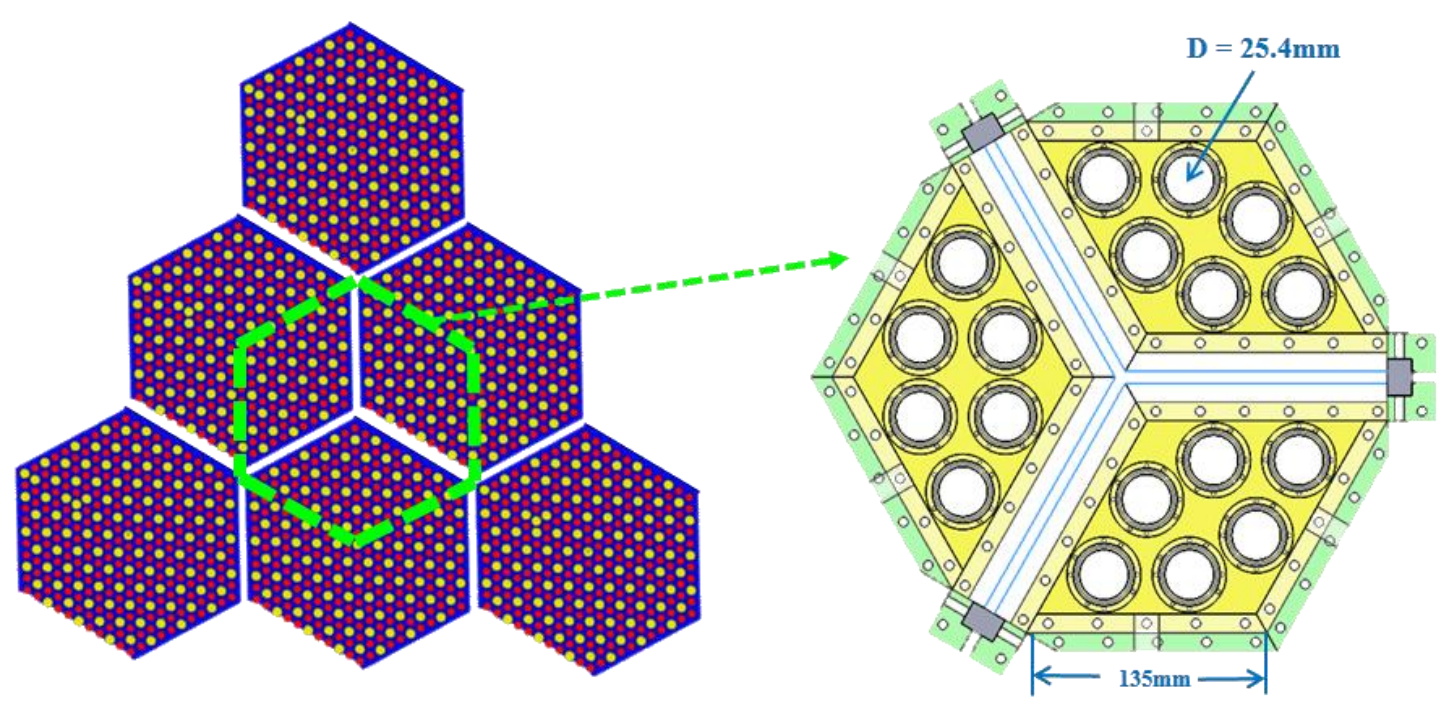

Fig. 6 Scale process from GA concept ${ }^{*}$ (left) to the TAMU model (right)

* This is just a representation of the GA concept fuel block but not the original GA design

Other important dimensions of the TAMU model are indicated in Fig. 7. The crossflow gap is not shown in this figure but can be seen in the CFD model mentioned in the next session. The facility is $1500 \mathrm{~mm}$ long including two layers of blocks and one layer for the collectors. Each layer is connected by flanges which are sealed with screws and gaskets. With collectors installed the coolant flow rate of each rhomboid can be measured either using Particle Image Velocimetry (PIV) technique or ultrasonic flow meter. After use of the total flow rate measured by a turbine flow meter, the bypass flow 
fraction can be calculated. Variation of the bypass flow gap width is achieved by employing different gap dividers shown in Fig. 7.
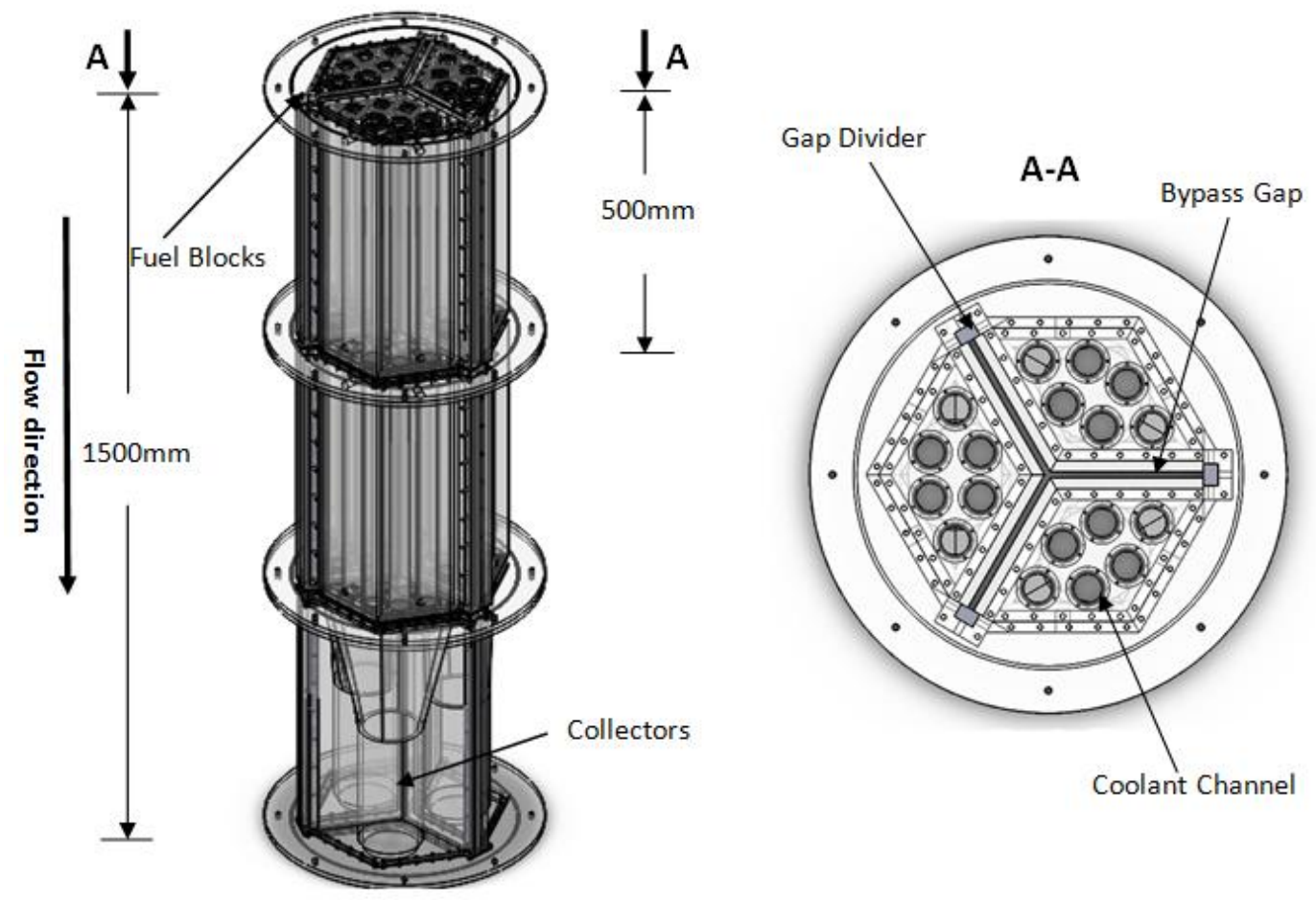

Fig.7 TAMU experimental facility

In order to match the Reynolds number in the real operation (estimated to be 30,000 at the medium power) an experimental loop was built as presented in Fig. 8 to increase the stability of the whole facility under high Reynolds number. The loop mainly consists of pump (7.5 KW, $3500 \mathrm{RPM})$, turbine flow meter, 500 gallon tank and three electronic flow meters. The main pipe is three inches connected by flexible valves. The 
sealing components in the loop are made of materials that are resistant to the P-cymene which is one of the working fluid. The loop is capable of providing of 250 gpm water which is equivalent to a Reynolds number of 38,524 in the coolant channel of the facility. In addition, several draining outlets were installed to prevent the working fluid like P-cymene from corroding the pipes and valves after the experiment is finished.

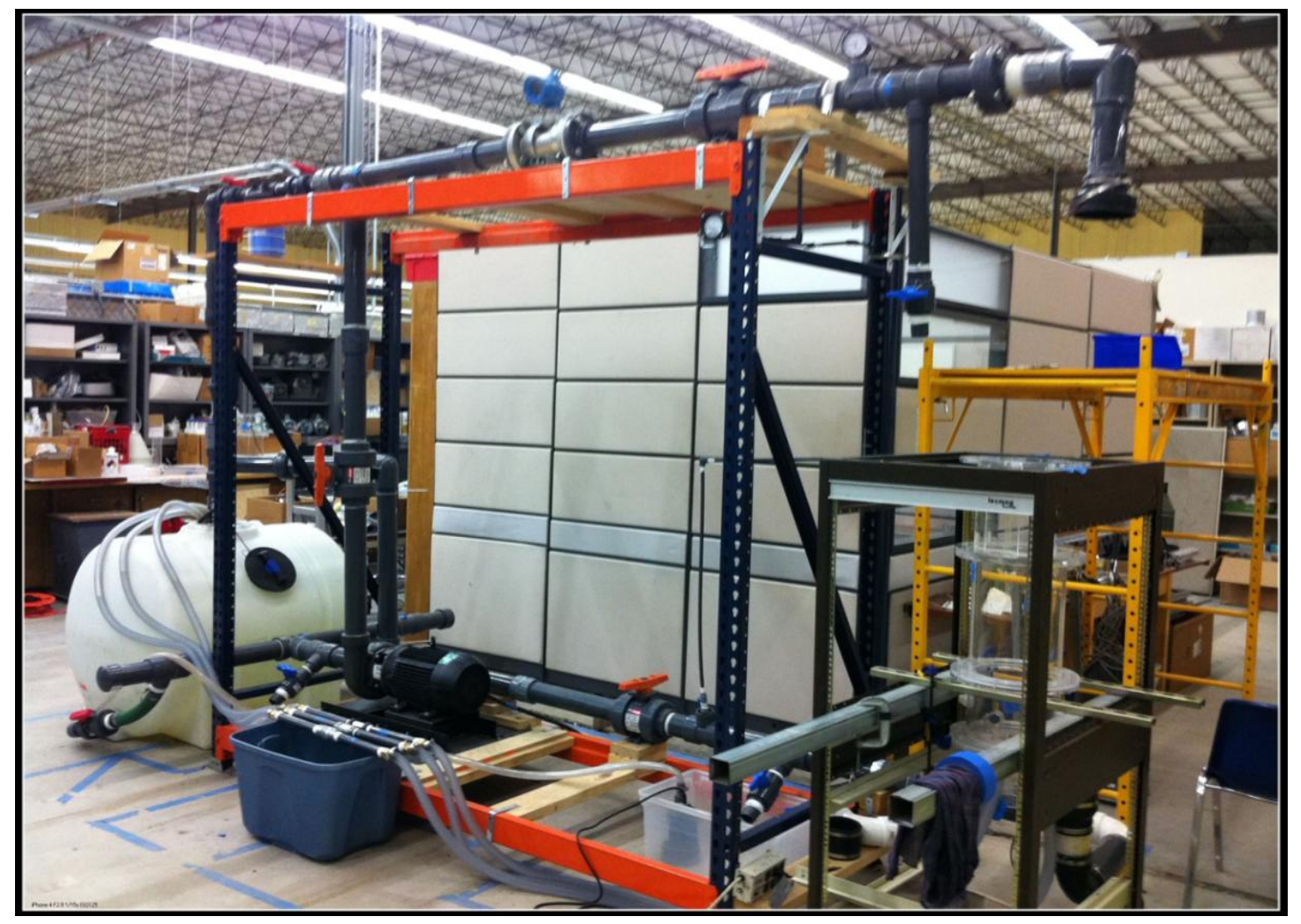

Fig.8 Experimental loop 


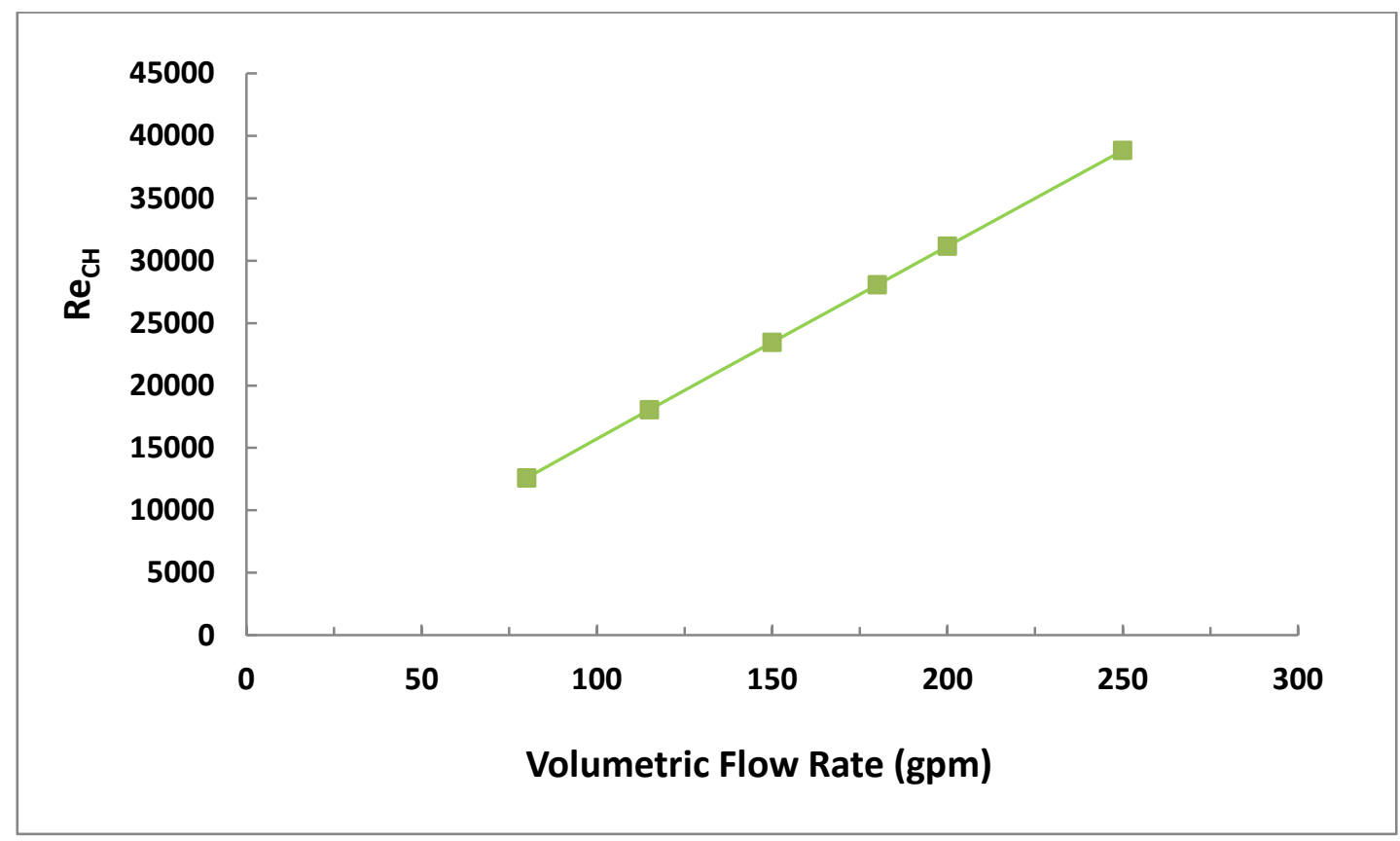

Fig.9 $\operatorname{Re}_{\mathrm{CH}}$ vs. Volumetric Flow Rate

where $\mathrm{Re}_{\mathrm{CH}}$ is the Reynolds number in the coolant channels.

$$
R e_{C H}=\frac{\rho D_{h} \bar{u}}{\mu}
$$

$\rho$ is the fluid density, $\mathrm{D}_{\mathrm{h}}$ is the hydraulic diameter, $\bar{u}$ is the average velocity and $\mu$ is the fluid dynamic viscosity.

Fig. 9 presents the Reynolds number based on the coolant channel as a function of the volumetric flow rate for the experimental facility. The facility is able to reach a Reynolds number that matches the real operational condition at an intermediate power level. In addition, it is clear that the flow in the coolant channels is turbulent. The working fluid here is water but not $\mathrm{P}$-cymene because it is not convenient to use $\mathrm{P}$ - 
cymene to test the loop since it is corrosive. Thus water is suitable to perform the preliminary test or experiment as well as corresponding CFD analysis. 


\section{CFD SIMULATIONS}

\subsection{Computational Domain}

Commercial CFD code STARCCM+ 6.04 was employed to perform all mesh generations and simulations in present study. A three dimensional geometry model which represents the computational or fluid region only was firstly built in Solidworks. Considering future challenge of the meshing work for the bypass gap and cross gap with a thickness of only several millimeters, each part of the model was created separately and was assembled later, which enables one to assign varied mesh size for different parts. With this multi-region meshing technique the meshing and computation will become more efficient and economical. A small drawback of this option is that a proper setup of interfaces is required to allow mass and momentum transferred among these parts. A stagnation box with a height of $150 \mathrm{~mm}$ is added to create a unified inlet condition for the coolant channels and bypass flow gap otherwise 19 inlets would need to be specified. Fig. 10 shows the computational model for the $6 \mathrm{~mm}$ bypass flow gap with $1 \mathrm{~mm}$ crossflow gap case. 


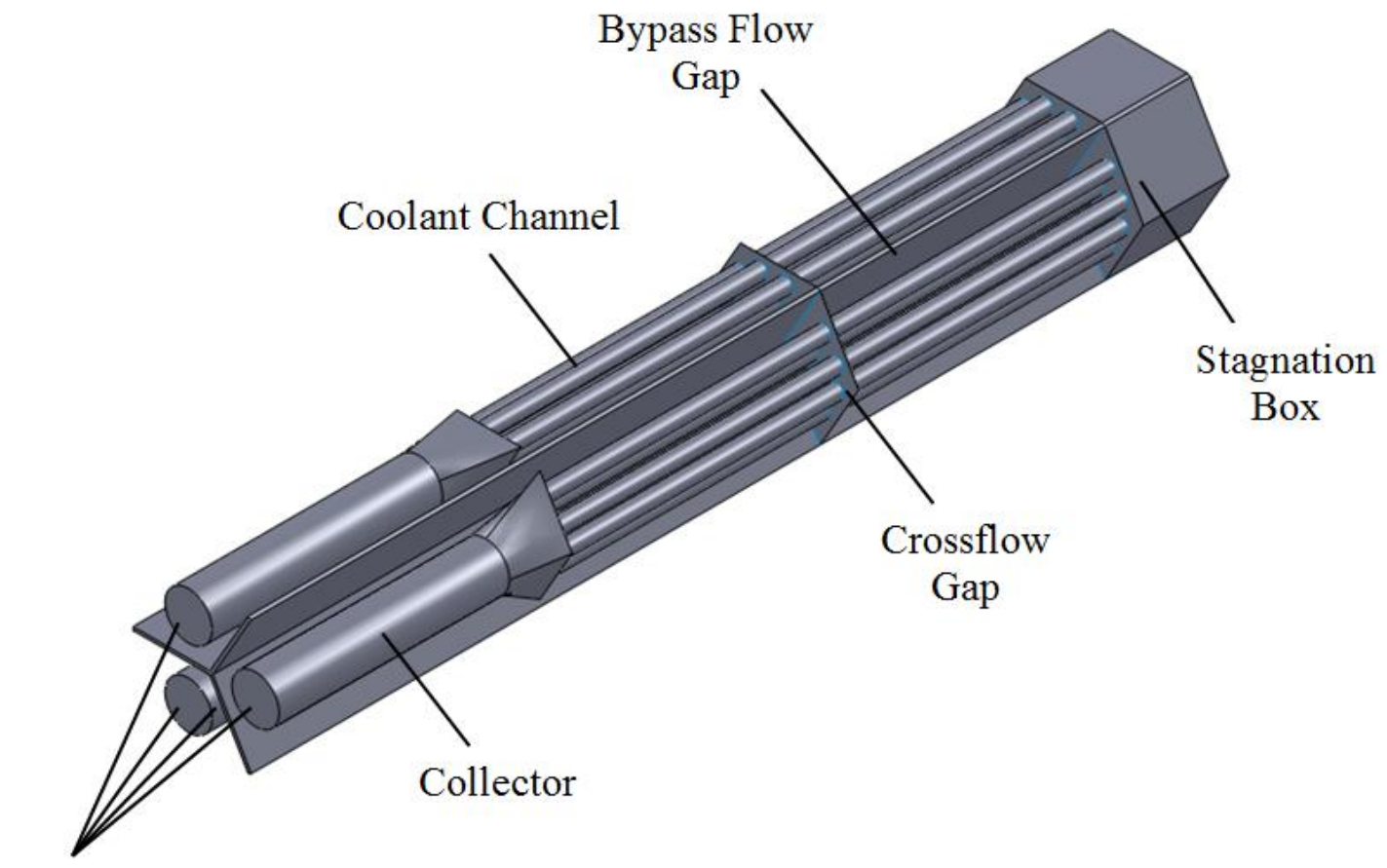

Outlets

Fig.10 Computational Domain

The model is mainly composed of six parts, stagnation box (inlet) and three outlets, coolant channel, crossflow gap, bypass flow gap and three collectors. The height of the stagnation box has been optimized so that the pressure loss can be reduced as much as possible but meanwhile a uniform inlet condition is achievable. The reason why the crossflow gap can be created as what is shown in Fig. 10 is the average mass flow rate within the crossflow gap is almost the same for each block when the system reaches equilibrium. Thus the crossflow gap can be isolated and treated as a part of the block itself. This assumption does not hold if there is net mass gain or loss for the crossflow 
gap because the mass cannot be conserved when the crossflow gap is isolated when the system has not reached steady state.

\subsection{Discretization}

The discretization is a process to subdivide the fluid domain into a number of smaller, non-overlapping subdomains called grid or mesh of cells. It is part of the first main element of any CFD codes, pre-processing and the other two elements are solving and post-processing (Versteeg, H. K., Malalasekera, W. 2007). For lots of flow problems the analytical solution is not obtainable and thus the differential governing equations needs to be converted to sets of algebraic or discretization equations so that they are able to be solved by computers. Mainly the governing equations for fluid flow are the mass and momentum conservation equations as shown in Eq. (2) and Eq. (3) if there is no heat transfer involved (Energy equation is not considered).

$$
\begin{gathered}
\frac{\partial \rho}{\partial t}=-(\nabla \cdot \rho \mathbf{v}) \\
\frac{\partial}{\partial t} \rho \mathbf{v}=-[\nabla \cdot \rho \mathbf{v v}]-\nabla p-[\nabla \cdot \tau]+\rho \mathbf{g}
\end{gathered}
$$

As mentioned above, the flow variable such as pressure $\mathrm{P}$ or velocity $\mathrm{V}$ in a continuous domain is defined at each grid point as $\mathrm{P}_{\mathrm{i}}$ or $\mathrm{V}_{\mathrm{i}}$. The values at other locations are obtained by interpreting the values at the grid points. Then the value of the flow variable at each grid is determined by solving this set of coupled algebraic equations in the discrete system. There are several popular discretization methods such as Finite 
Difference Method (FDM), Finite Element Method (FEM) and Finite Volume Method (FVM) which is employed in the code STARCCM+. For the FVM, the continuous fluid domain is divided into a number of control volumes or cells and the flow variables are located at the center of these control volumes. By integrating the differential form of the governing equations over the each control volume the discretization equation is obtained. The largest advantage of the FVM is the resulting solution conserves mass, momentum or energy.

There are three mesh models provided in STARCCM+, tetrahedral mesh, trimmer mesh (hexahedra) and polyhedral mesh. Since multi-region meshing is not available under trimmer mesh model, only tetrahedra and polyhedra were taken into consideration. The advantage of tetrahedral cells is the fast speed to generate and low cost of memory. However, since there are only four neighbors around one control volume and also it's possible that these neighbors are located almost in the same plane, it would be difficult to capture the velocity gradient accurately. In contrast, each polyhedral cell has more than 10 neighbors from which more accurate results can be obtained. Also, polyhedra mesh needs less cells than that for the tetrahedral meshes, which means less computational hours consumption (M. Peric, et al, 2005). Table 2 indicates the details of some important parameters for the mesh generation for the case of bypass gap $6 \mathrm{~mm}$ with $1 \mathrm{~mm}$ cross gap abbreviated as BG6CG1. Default values were chosen for the rest. 
Table 2 Summary of Parameters for Mesh Generation (Case BG6CG1)

\begin{tabular}{|c|c|}
\hline Parameter & Value \\
\hline Globe Base Size(mm) & 3 \\
\hline Number of Prism Layers & 1.2 \\
\hline Prism Layer Stretching & 1 \\
\hline Prism Layer Thickness(mm) & 1.3 \\
\hline Surface Growth Rate & 3 \\
\hline Base Size for Bypass Gap(mm) & 0.3 \\
\hline Base Size for Cross Gap(mm) & \\
\hline
\end{tabular}

Fig. 11 presents the multi-region mesh technique employed in this work. The fluid domain is divided into three regions in which different mesh densities are specified. The crossflow gap region characterizes for a very thin thickness so more cells are created to capture the flow profile within the narrow gap. Compared to the narrow gaps, the main body has a relative larger volume so low density meshes are adequate to obtain accurate results with respect to the computation economy. 


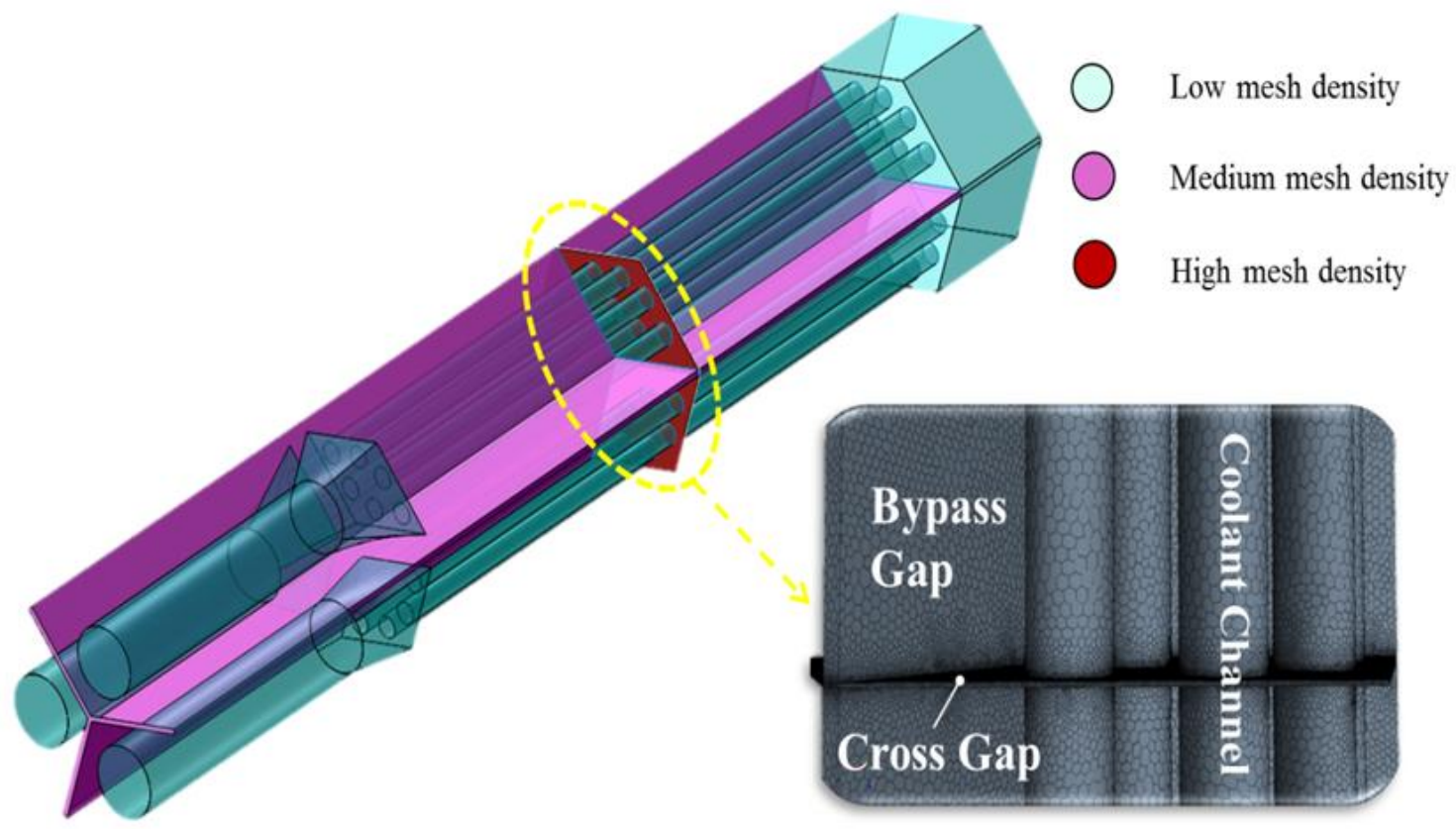

Fig.11 Illustration of the multi-region meshing technique

Another concern regarding the multi-region meshing is the mesh condition on the interfaces. Generally a significant error may be introduced if the mesh on the interface is non-conformal meaning that the two faces on the interfaces are meshed with different sizes of elements given that two parts are connected tightly without any rotation. Thus at most of the time the conformal is mesh is preferred. Fig. 12 shows the conformal mesh generated in the present study. 


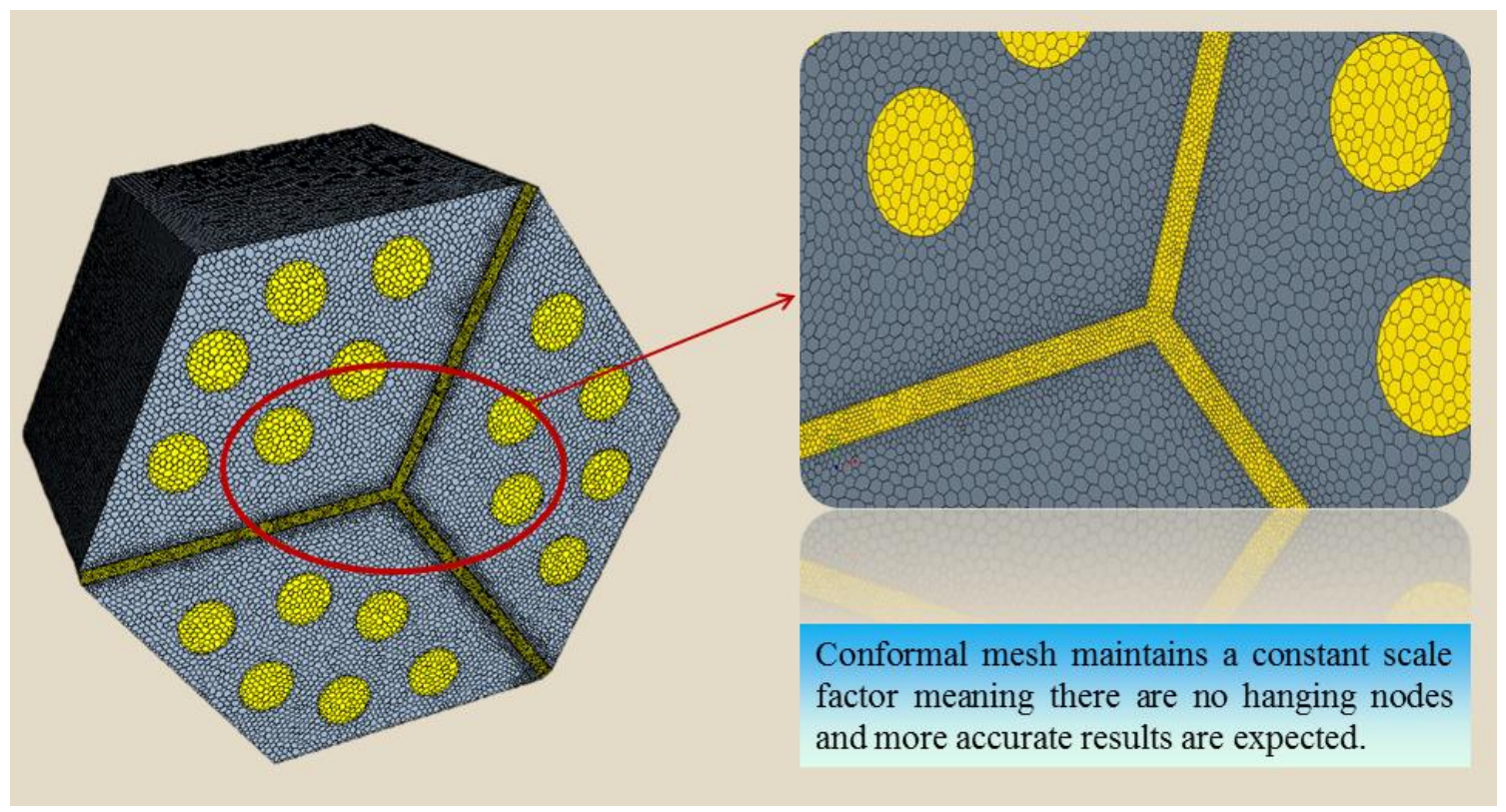

Fig.12 Illustration of conformal Mesh on the interface of the stagnation box and the bypass gap

\subsection{Physics Models and Boundary Conditions}

\subsubsection{Differencing Scheme}

STARCCM+ is a control-volume based computational technique which integrates the governing equations for each single control volume. In present work, in order to improve the computational accuracy the second-order upwind scheme was used to calculate the convective terms at the interface of each cell. Although the first order upwind scheme was not selected, it still worths a discussion of the algorithm to have a better understanding of the second order upwind scheme which is more widely used in CFD calculations. Given a diffusion-convection problem, the one-dimension control volume is illustrated in Fig. 13. 


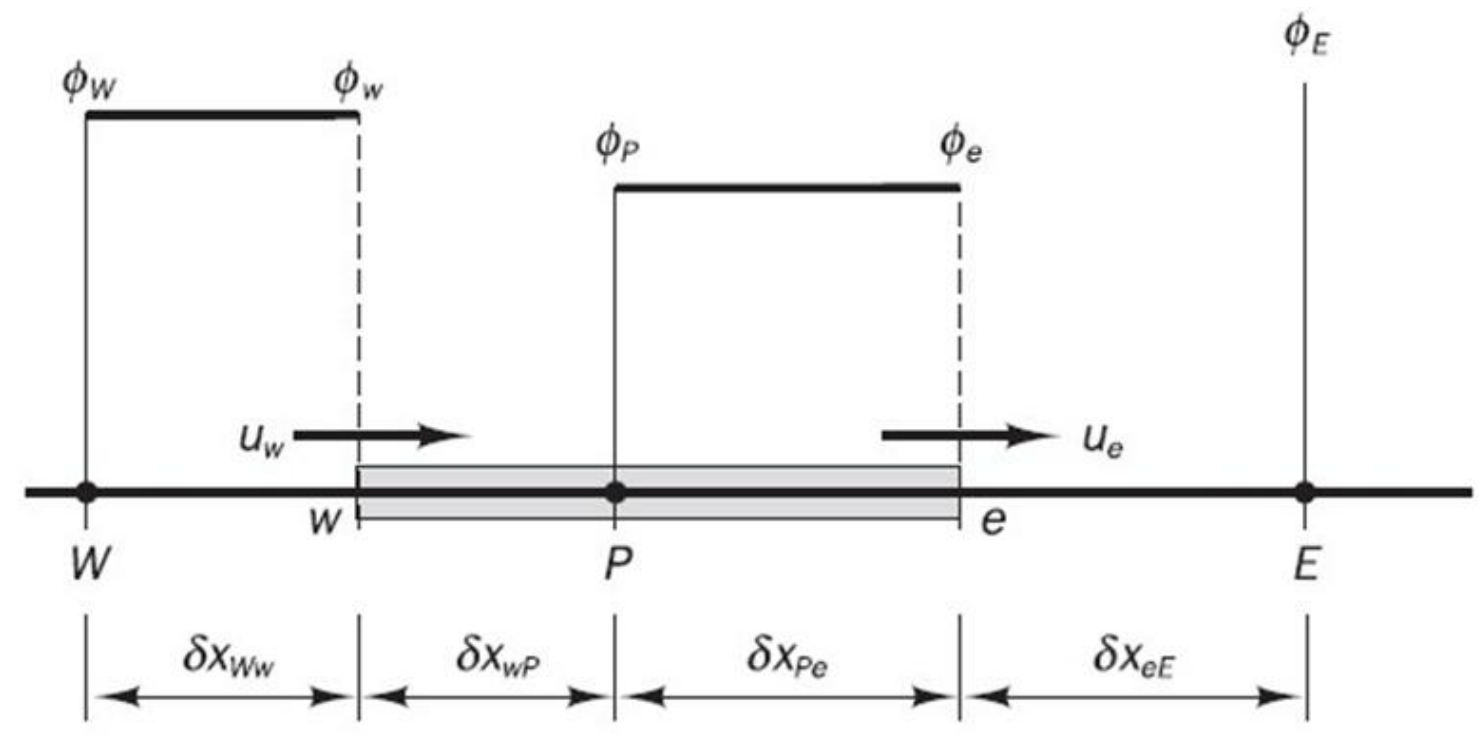

Fig.13 Control volume for the one-dimension diffusion-convection problem (Versteeg, H. K., Malalasekera, W. 2007)

The flow viable $\phi$ can be velocity or pressure as mentioned in previous chapter. The upper-case letters (W, P, and E) stand for the control volume centroid while the lower-case letter such as $\mathrm{w}$ and e represent the value at the cell faces. The fluid flows from the west to the east passing through the midpoint $\mathrm{P}$ between $\mathrm{W}$ and $\mathrm{E}$. To calculate the value at the cell faces, the first order upwind scheme sets the value of $\phi$ at the cell face or interface $\left(\phi_{w}\right.$ or $\left.\phi_{e}\right)$ to be equal to the value of $\phi$ at the grid point $\left(\phi_{W}\right.$ or $\left.\phi_{P}\right)$ on the upwind side of the interface. That is,

$$
\begin{aligned}
& \phi_{w}=\phi_{w} \text { when } F_{w}>0 \\
& \phi_{e}=\phi_{P} \quad \text { when } F_{e}>0
\end{aligned}
$$

where

$$
\begin{aligned}
& F_{w}=\rho U_{w} \\
& F_{e}=\rho U_{e}
\end{aligned}
$$


$\mathrm{U}$ is the velocity and F represents the strength of the convection of flow (S.V. Patankar, 1980). The accuracy of this scheme can be evaluated by the Taylor series analysis.

$$
\phi(x)=\phi_{P}+\left(x-x_{P}\right) \frac{\partial \phi}{\partial x}+\frac{\left(x-x_{P}\right)^{2}}{2 !} \frac{\partial^{2} \phi}{\partial x^{2}}+O(\Delta x)^{3}
$$

Now it is clear to see that the scheme is a first-order algorithm based on the backward differencing scheme. Thus it is not adequate for calculations which require high accuracy without significant false diffusion. Second-order Upwind Differencing Scheme with fine mesh will dampen this drawback. The value of $\phi$ is determined from the cell values in the two cells upstream of the face as illustrated in Fig. 14. It can be seen that the value of $\phi_{e}$ is calculated based on a linear interpolation. Assuming the length of segment WP is the same as that of PE, $\phi_{e}$ is

$$
\phi_{e}=\phi_{P}+\frac{\phi_{P}-\phi_{W}}{2}
$$

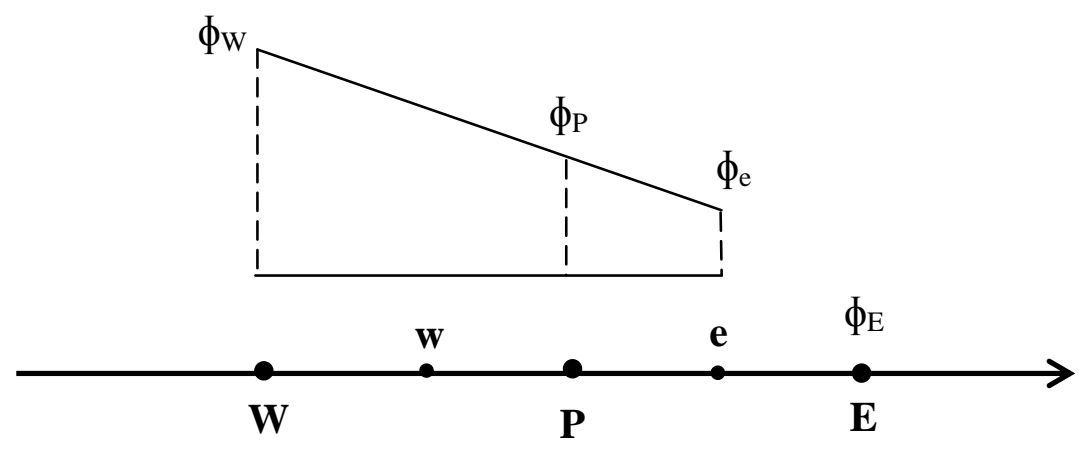

Fig.14 Second-order upwind differencing scheme 


\subsubsection{Turbulence Modeling}

Turbulent flow is characterized by its irregularity, diffusivity, unsteadiness and dissipation. The energy is transferred by stretching and rotation of large eddies to smaller eddies where the kinetic energy is eventually dissipated into heat. The difficulties of describing turbulence lie in the fact that instantaneous contributions to the flows can vary capriciously as a function of time. Fortunately, it is found that all variables in a flow can be decomposed into a time-averaged value $(\bar{v})$ and a fluctuating $\operatorname{part} v^{\prime}$

$$
v=\bar{v}+v^{\prime}
$$

which is called Reynolds decomposition. By substituting Eq. (8) into the Navier-Stokes equations, it gives the so-called Reynolds-averaged Navier-Stokes (RANS) equations presented as below (viscosity is constant).

X-component of RANS

$$
\begin{aligned}
\frac{\partial}{\partial t}(\rho \bar{u})+\frac{\partial}{\partial x} & (\rho \bar{u} \bar{u})+\frac{\partial}{\partial y}(\rho \bar{v} \bar{u})+\frac{\partial}{\partial z}(\rho \bar{w} \bar{u}) \\
= & -\frac{\partial \bar{P}}{\partial x}+\mu\left(\frac{\partial^{2} \bar{u}}{\partial x^{2}}+\frac{\partial^{2} \bar{u}}{\partial y^{2}}+\frac{\partial^{2} \bar{u}}{\partial z^{2}}\right)-\frac{\partial}{\partial x_{i}}\left(\rho \overline{u^{\prime} u_{\imath}^{\prime}}\right)
\end{aligned}
$$

Y-component of RANS

$$
\begin{aligned}
\frac{\partial}{\partial t}(\rho \bar{v})+\frac{\partial}{\partial x} & (\rho \bar{u} \bar{v})+\frac{\partial}{\partial y}(\rho \bar{v} \bar{v})+\frac{\partial}{\partial z}(\rho \bar{w} \bar{v}) \\
& =-\frac{\partial \bar{P}}{\partial y}+\mu\left(\frac{\partial^{2} \bar{v}}{\partial x^{2}}+\frac{\partial^{2} \bar{v}}{\partial y^{2}}+\frac{\partial^{2} \bar{v}}{\partial z^{2}}\right)-\frac{\partial}{\partial x_{i}}\left(\rho \overline{v^{\prime} u_{\imath}^{\prime}}\right)
\end{aligned}
$$

Z-component of RANS 


$$
\begin{aligned}
\frac{\partial}{\partial t}(\rho w)+\frac{\partial}{\partial x} & (\rho \bar{u} \bar{w})+\frac{\partial}{\partial y}(\rho \bar{v} \bar{w})+\frac{\partial}{\partial z}(\rho \bar{w} \bar{w}) \\
& =-\frac{\partial \bar{P}}{\partial z}+\mu\left(\frac{\partial^{2} \bar{z}}{\partial x^{2}}+\frac{\partial^{2} \bar{z}}{\partial y^{2}}+\frac{\partial^{2} \bar{z}}{\partial z^{2}}\right)-\frac{\partial}{\partial x_{i}}\left(\rho \overline{w^{\prime} u^{\prime}}\right)
\end{aligned}
$$

The term $\rho \overline{u^{\prime} u^{\prime}}$ is a compact tensor and is usually referred to as the Reynolds stresses. The Reynolds stresses are complicated functions of the position and the turbulence intensity (Bird, R. B, Stewart, W.E., et al, 2006). Thus empirically correlations or models need to be introduced to resolve the Reynolds stresses term and this process is called turbulence modeling. The realizable k- $\varepsilon$ turbulence model used in the present study is one of the most widely used models because of its robustness and low computational cost with respect to a decent accuracy for varied flow patterns. "Realizable" here means that the model satisfies some constraints that are consistent with the physics of turbulence (T. H. Shih et al, 1995).

Turbulence kinetic energy (k) equation:

$$
\nabla \cdot(\rho V k)=\nabla \cdot\left[\left(\mu+\frac{\mu_{T}}{\sigma_{k}}\right) \nabla k\right]+\mu_{T} S^{2}-\rho \varepsilon
$$

Specific dissipation rate equation:

$$
\nabla \cdot(\rho V \varepsilon)=\nabla \cdot\left[\left(\mu+\frac{\mu_{T}}{\sigma_{\varepsilon}}\right) \nabla \varepsilon\right]+C_{1} \rho S_{\varepsilon}-C_{2} \rho \frac{\varepsilon^{2}}{k+\sqrt{v \varepsilon}}
$$

Where

$$
\begin{aligned}
C_{1} & =\max \left(0.43, \frac{\eta}{\eta+5}\right) \\
\mathrm{C}_{2} & =1.9
\end{aligned}
$$

Eddy viscosity: 


$$
\mu_{T}=\rho C_{\mu} \frac{k^{2}}{\varepsilon}
$$

Instead of constant in the standard k- $\varepsilon$ model, $C_{\mu}$ in the realizable k- $\varepsilon$ model is computed from:

$$
C_{\mu}=\frac{1}{A_{0}+A_{s} U^{*} \frac{k}{\varepsilon}}
$$

Where

$$
\begin{aligned}
A_{0}=4.0 & \\
U^{*} & =\sqrt{S_{i j} S_{i j}+\Omega_{i j} \Omega_{i j}} \\
A_{s} & =\sqrt{6} \cos \left(\frac{1}{3} \arccos (\sqrt{6} W)\right) \\
W & =\frac{\sqrt{8} S_{i j} S_{j k} S_{k i}}{S^{3}} \\
\text { Vorticity tensor } \quad \Omega_{i j} & =\frac{1}{2}\left(\frac{\partial \overline{u_{l}}}{\partial \overline{\bar{x}_{j}}}-\frac{\partial \overline{u_{j}}}{\partial \bar{x}_{l}}\right)
\end{aligned}
$$

\subsubsection{Near Wall Treatment}

The presence of walls will significantly affect the turbulent flows because of the existence of large gradients in the flow variables in the near-wall regions. The gradients are produced due to the fact that the non-slip condition holds and the fluid is viscous. In addition, the $\mathrm{k}-\varepsilon$ turbulence model is valid away from the walls. Thus special treatment for the near-wall region is necessary. Traditionally, the near-wall region can be subdivided into three sub-regions as shown in Fig. 15. 


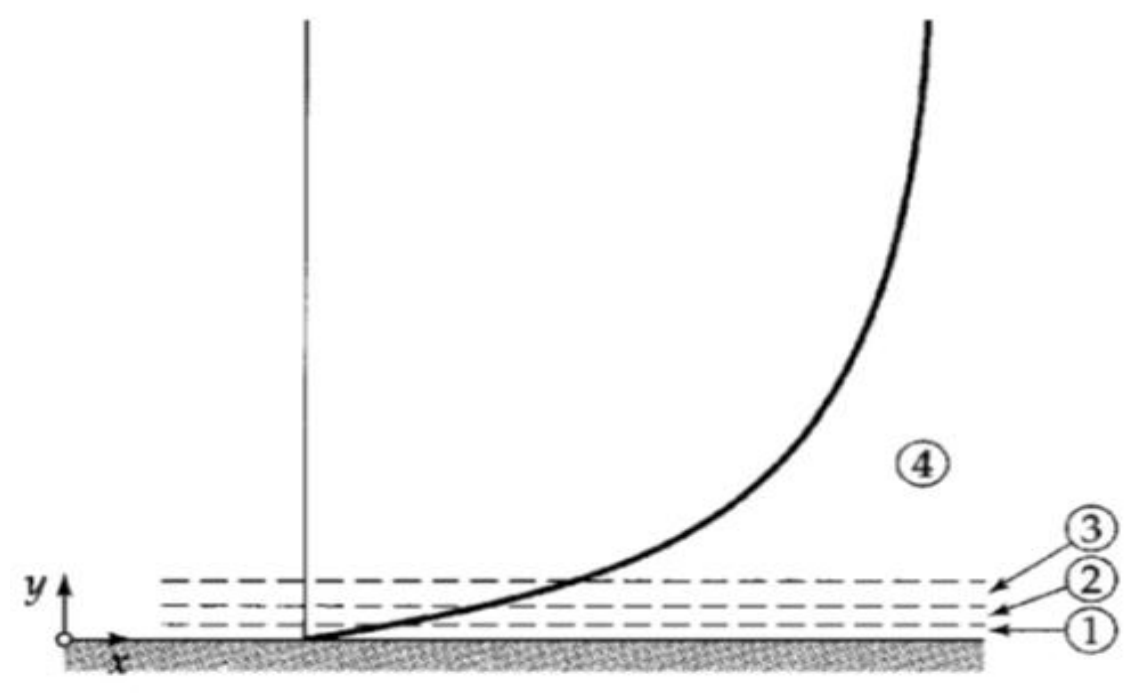

1. Viscous sublayer $\left(0<\mathrm{y}^{+}<5\right)$;

2. Buffer layer $\left(5<\mathrm{y}^{+}<60\right)$;

3. Inertial sublayer (logarithmic layer, $60<\mathrm{y}^{+}<300$ );

4. Main turbulent stream $\left(\mathrm{y}^{+}>300\right)$

Fig.15 Subdivisions of the near-wall region for the turbulent flow

(Bird, R. B, Stewart, W.E., et al, 2006)

Here the $\mathrm{y}^{+}$is a non-dimensional wall distance defined in Eq. (20) to evaluate the grid resolution near the wall.

$$
y^{+}=\frac{u_{*} y}{v}
$$

Where $u_{*}$ is the friction velocity defined as $u_{*}=\sqrt{\frac{\tau_{w}}{\rho}}, \tau_{w}$ is the wall shear stress and $\rho$ is the fluid density, $\mathrm{y}$ is the physical distance to the nearest wall and $v$ is the kinetic viscosity. 
There are mainly two approaches to model the flow in the near-wall regions. One is called wall function approach which employs semi-empirical wall functions to connect the viscosity-affected region between the wall and the main turbulent region. This approach does not resolve the viscous sublayer and buffer layer. In contrast, the other approach, the near-wall model approach, requires very fine mesh in the near-wall region to resolve the flow in the inner region including the viscous sublayer and buffer layer (FLUENT, 2009). These two approaches are illustrated in Fig. 16.

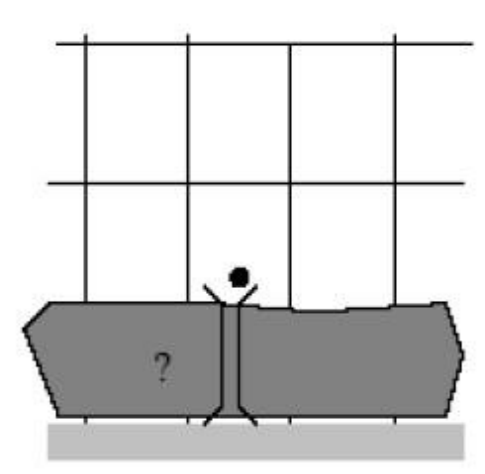

Wall Function Approach

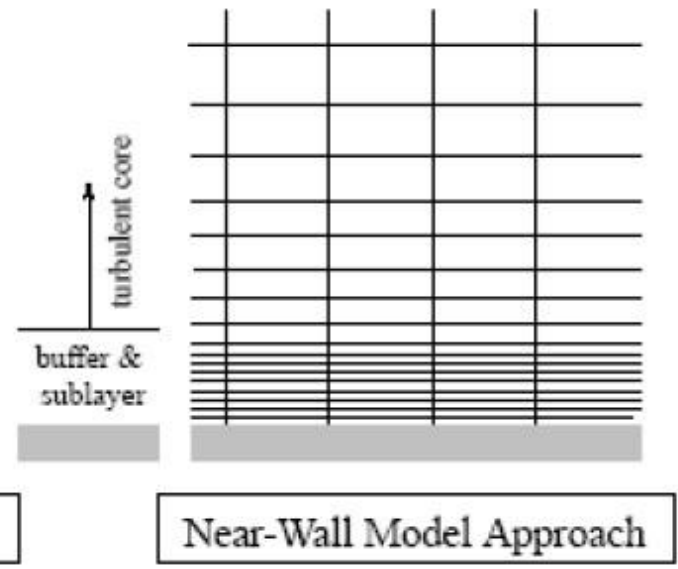

Near-Wall Model Approach

Fig.16 Two approaches for the wall treatment (FLUENT, 2009)

Obviously, the near-wall model approach is more accurate since fine meshes are created all the way down to the wall. However, the increase of cells in the near-wall region will dramatically increase the total number of cells in the whole domain, which decreases the computational economy. Considering the focus of the present study does not lie in the study of the flow near the wall, the wall function approach is employed. 
The two-layer all $\mathrm{y}^{+}$wall treatment was used in the present study to blend the wall laws to estimate the shear stress. It is a hybrid treatment that attempts to emulate the high $\mathrm{y}^{+}$ wall treatment for coarse meshes and the low $\mathrm{y}^{+}$wall treatment for the fine meshes. It also provides satisfactory answers for the intermediate meshes (STARCCM+, 2011). Although the wall function approach does not require very fine mesh in the near-wall region, coarse meshes will fail to capture the large gradients in the flow near the wall accurately. Five prism layers were chosen with a stretching ratio of 1.2, which produces a reasonable local $\mathrm{y}^{+}$value (within the logarithmic layer) in the interested regions. The following equations were used to estimate the value of $\mathrm{y}^{+}$. Eq. (20) can be rewritten as:

$$
y^{+}=\frac{u_{*} y \rho}{\mu}
$$

Where $\mu$ is the fluid dynamic viscosity, $\rho$ is the fluid density.

$$
u_{*}=\sqrt{\frac{\tau_{w}}{\rho}}
$$

Shear Stress

$$
\tau_{w}=C_{f} \frac{1}{2} \rho U_{\text {freestream }}^{2}
$$

Where skin friction coefficient can be estimated using Schlichting skin-friction correlation:

$$
C_{f}=\left[2 \log _{10}\left(R e_{x}\right)-0.65\right]^{-2.3}, \text { for } \mathrm{Re}_{\mathrm{x}}<10^{9}
$$

Reynolds number $\quad R e=\frac{\rho U_{\text {freestream }} L_{\text {boundarylayer }}}{\mu}$

For a pipe flow, the free stream velocity can be treated as average velocity which is calculated using the known mass flow rate and the area of the cross section. The characteristic length $\mathrm{L}_{\text {boundary layer }}$ is the thickness of the boundary layer at a certain 
distance $\mathrm{x}$ away from the inlet of the flow. This thickness can be approximately

evaluated using Eq. (26) (R. W. Fox, 2006) which is, however, is mainly to estimate the boundary thickness for the turbulent flow over a flat plate.

$$
\delta \approx \frac{0.382 x}{R e_{x}^{\frac{1}{5}}}
$$

It was found that the $\mathrm{y}^{+}$value in present work ranges from 30 to 150 depends on the position selected. Thus the first prism layer is located within the buffer layer or inertial layer (logarithmic layer) which is adequate to employ the two-layer all $\mathrm{y}^{+}$wall treatment (STARCCM+, 2011).

\subsubsection{Setup for Other Models}

Gravity was considered in the calculation since it would be more realistic and easy for one to compare with experimental results at least for liquid. The fluid was assumed to be incompressible and isothermal. The current simulation adapted the three dimensional steady state models as the outlets of the facility are basically three long cylindrical pipes where no significant recirculation would be expected and a steady state solution could be obtained.

\subsubsection{Boundary Conditions}

The inlet boundary conditions were specified as the uniform mass flow rate condition and the values vary from $80 \mathrm{gpm}(5.0 \mathrm{~kg} / \mathrm{s})$ to $250 \mathrm{gpm}(15.8 \mathrm{~kg} / \mathrm{s})$. The zero pressure (gauge pressure) outlet boundary was applied to the bypass gap outlet and three 
coolant collector outlets. The intensity + turbulence length scale was used in the option of turbulence specification. In pipe flows, the turbulence length scale (TLS) was estimated using Eq. (27):

$$
\mathrm{L}=0.07 \mathrm{D}_{\mathrm{h}}
$$

Where $\mathrm{L}$ is the turbulence length scale and $\mathrm{D}_{\mathrm{h}}$ is hydraulic diameter.

\subsection{Grid Independence Study}

The Reynolds-averaged Navier-Stokes (RANS) equations are solved by discretizing the fluid domain so that the partial differential equations can be represented by sets of algebraic equations. This gives rise to recognized numerical errors including discretization errors. The other two errors are roundoff errors and iterative convergence errors (Versteeg, H. K., Malalasekera, W. 2007) which will be discussed in the uncertainly analysis chapter.

\subsubsection{Discretization Errors vs. Grid Resolution}

For a flow variable $\phi(x)$ the Taylor series expansion $\left(2^{\text {nd }}\right.$ order $)$ around point $\mathrm{x}$ is

$$
\phi(x+\Delta x)=\phi(x)+\left(\frac{\partial \phi}{\partial x}\right)_{x} \Delta x+\left(\frac{\partial^{2} \phi}{\partial x^{2}}\right)_{x} \frac{\Delta x^{2}}{2}+O\left(\Delta x^{3}\right)
$$

So the truncated term $O\left(\Delta x^{3}\right)$ or $O\left(\Delta x^{n}\right)$ in general is the source of the discretization errors when the fluid domain is cut into small pieces. Apparently this truncation error or the discretization error is proportional to the grid spacing or inversely proportional to the mesh density or gird resolution. The relationship between discretization error and the number of cells are qualitatively illustrated in Fig. 17. 


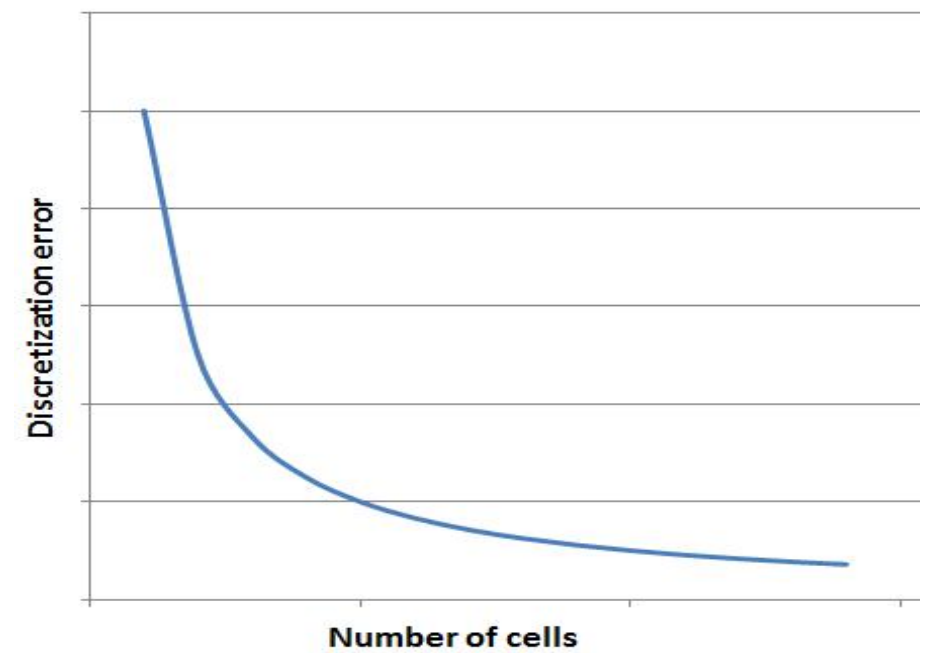

Fig.17 Discretization error vs. Number of cells

However, larger cell densities do not guaranty smaller errors because the roundoff error will be significant as the mesh sizes become very small. Thus constrains here are only the computational cost but also the roundoff errors when the mesh sizes are small. In order to investigate the influence to the results from the sizes of the mesh a grid independence study is necessary. A solution which is not mesh independent is always preferred.

\subsubsection{Richardson Extrapolation Approach}

Although the application of CFD has helped engineers save time and money in design and product improvement and also have a deeper understanding of the flow characteristics without building complex experimental facilities, inaccurate results will be a waste time and effort and even cause a failure of products. Obviously sensitivity 
study will provide engineers a better understanding and help the industry obtain a more accurate estimation of any limits of their products. There are a number of guidelines for best practice in CFD (Versteeg, H. K., Malalasekera, W. 2007) such as AIAA (1998) and ERCOFTAC (2000) guidelines. After years of development these guidelines have been revised many times to meet the new requirements. In recent years guidelines from the Journal of Fluid Engineering recommend that the Grid Convergence Indices (GCI) Method based on the Richardson extrapolation (RE) method can be used for estimation of the discretization error. The main procedure is briefly described below (Celik, I.B., Ghia, U, et al, 2008):

Step 1. A representative mesh size $\mathrm{h}$ is defined (three dimensions case).

$$
h=\left[\frac{1}{N} \sum_{i=1}^{N}\left(\Delta V_{i}\right)\right]^{\frac{1}{3}}
$$

where $\Delta V_{i}$ is the volume of $\mathrm{i}_{\text {th }}$ domain and $\mathrm{N}$ is the total number of cells. In present study, $\mathrm{h}$ is treated as the global mesh size.

Step 2. Generate three sets of meshes with different grid densities, for example, low, medium and high. The ratio $\mathrm{r}=\mathrm{h}_{\text {coarse }} / \mathrm{h}_{\text {fine }}$ should be greater than 1.3.

Step 3. Calculate the apparent order $\mathrm{p}$ of the method using the expression

$$
\begin{gathered}
p=\frac{1}{\ln \left(r_{21}\right)}|\ln | \varepsilon_{32} / \varepsilon_{21}|+\mathrm{q}(\mathrm{p})| \\
q(p)=\ln \left(\frac{r_{21}^{p}-s}{r_{32}^{p}-s}\right) \\
s=1 \cdot \operatorname{sgn}\left(\frac{\varepsilon_{32}}{\varepsilon_{21}}\right)
\end{gathered}
$$


where $\mathrm{r}_{21}=\mathrm{h}_{2} / \mathrm{h}_{1}, \mathrm{r}_{32}=\mathrm{h}_{3} / \mathrm{h}_{2}$, here $\mathrm{h}_{1}<\mathrm{h}_{2}<\mathrm{h}_{3}, \varepsilon_{32}=\Phi_{3}-\Phi_{2}, \varepsilon_{21}=\Phi_{2}-\Phi_{1}$, and $\Phi_{k}$ is the solution of any flow variables that is important. Eq. (30) - (32) can be solved using fixed-point iteration, with the initial guess equal to the first term.

Step 4. The p in Eq. (31) will be used to calculate the extrapolated values by

$$
\begin{aligned}
& \Phi_{\text {ext }}^{21}=\left(r_{21}^{p} \Phi_{1}-\Phi_{2}\right) /\left(r_{21}^{p}-1\right) \\
& \Phi_{\text {ext }}^{32}=\left(r_{32}^{p} \Phi_{3}-\Phi_{2}\right) /\left(r_{32}^{p}-1\right)
\end{aligned}
$$

Step 5. Error can be estimated by

Approximate relative error,

$$
e_{a}^{21}=\left|\frac{\Phi_{1}-\Phi_{2}}{\Phi_{1}}\right|
$$

Extrapolated relative error,

$$
e_{\text {ext }}^{21}=\left|\frac{\Phi_{\text {ext }}^{12}-\Phi_{1}}{\Phi_{\text {ext }}^{12}}\right|
$$

The fine-grid convergence index,

$$
G C I_{\text {fine }}^{21}=\frac{1.25 e_{a}^{21}}{r_{21}^{p}-1}
$$

In present study, three meshes with different mesh densities were generated for three cases, bypass flow gaps of $6 \mathrm{~mm}, 4 \mathrm{~mm}$ and $2 \mathrm{~mm}$. The crossflow gap is uniform and the width is $1 \mathrm{~mm}$ for all three cases. The mesh sizes are summarized in Table 3 . In order to maintain a relatively high meshing efficiency only the global base size and the crossflow gap base size were kept constant for three cases. It is not reasonable to set the base size of bypass flow gap to be the same. For example, the base size of the bypass flow gap is $3 \mathrm{~mm}$ for the coarse mesh (BG6CG1 case) but this dimension cannot be 
applied to the BG2CG1 case since the base size itself is larger than the bypass flow gap size which is $2 \mathrm{~mm}$. Thus it is still reasonable and acceptable to use global base size to perform the grid independence study and to investigate the corresponding errors based on the Richardson extrapolation.

Table 3 Summary of cases investigated for the grid independence study

\begin{tabular}{|c|c|c|c|c|}
\hline Case & $\begin{array}{c}\text { Global base size } \\
(\mathrm{mm})\end{array}$ & $\begin{array}{c}\text { BG base size } \\
(\mathrm{mm})\end{array}$ & $\begin{array}{c}\text { CG base size } \\
(\mathrm{mm})\end{array}$ & $\begin{array}{c}\text { Number of cells } \\
(\text { million })\end{array}$ \\
\hline \multirow{3}{*}{ BG6CG1 } & 8.00 & 3.00 & 0.50 & 4.76 \\
\cline { 2 - 5 } & 6.00 & 2.30 & 0.35 & 9.50 \\
\cline { 2 - 5 } & 4.50 & 1.70 & 0.26 & 18.32 \\
\hline \multirow{3}{*}{ BG4CG1 } & 8.00 & 2.00 & 0.50 & 5.77 \\
\cline { 2 - 5 } & 6.00 & 1.50 & 0.35 & 11.56 \\
\hline \multirow{3}{*}{ BG2CG1 } & 4.50 & 1.10 & 0.26 & 22.57 \\
\cline { 2 - 5 } & 8.00 & 1.00 & 0.50 & 11.14 \\
\cline { 2 - 5 } & 6.00 & 0.75 & 0.35 & 22.21 \\
\hline
\end{tabular}

Note: BG - Bypass flow Gap, CG - Crossflow Gap

\subsubsection{Results of the Grid Independence Study}

Forty eight steady state simulations were run to investigate the influence to the solution from the grid base size. The bypass flow fraction was selected as the crucial variable which is the $\Phi$ mentioned in the section 3.4.2. For each size of the bypass flow 
gap, three different meshing densities were specified, low, intermediate and high. Fig. 18- Fig. 20 presents the bypass flow fraction as a function of volumetric flow rate for each bypass flow gap size.

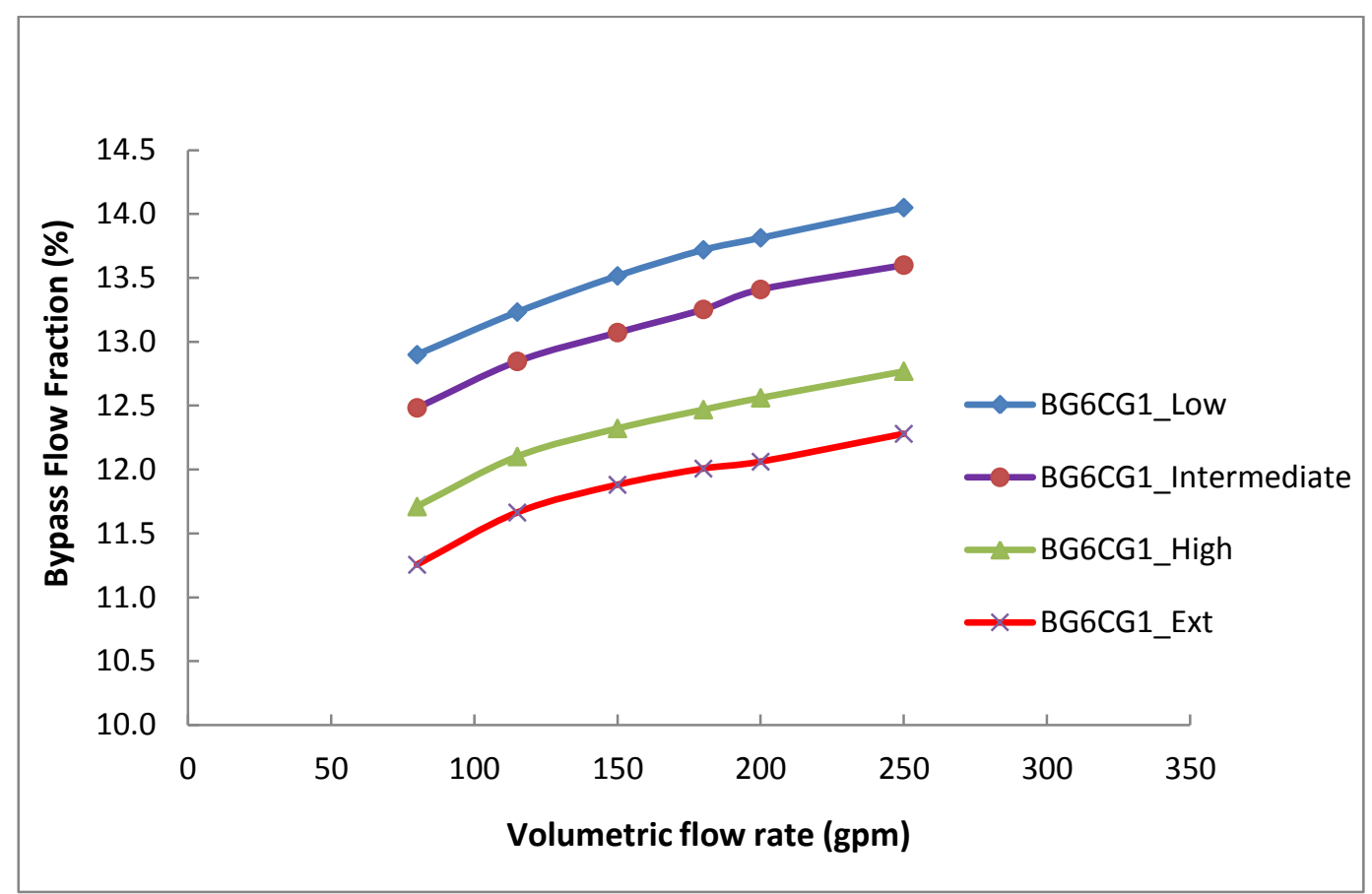

Fig.18 Bypass flow fraction vs. Volumetric flow Rate (BG6CG1) 


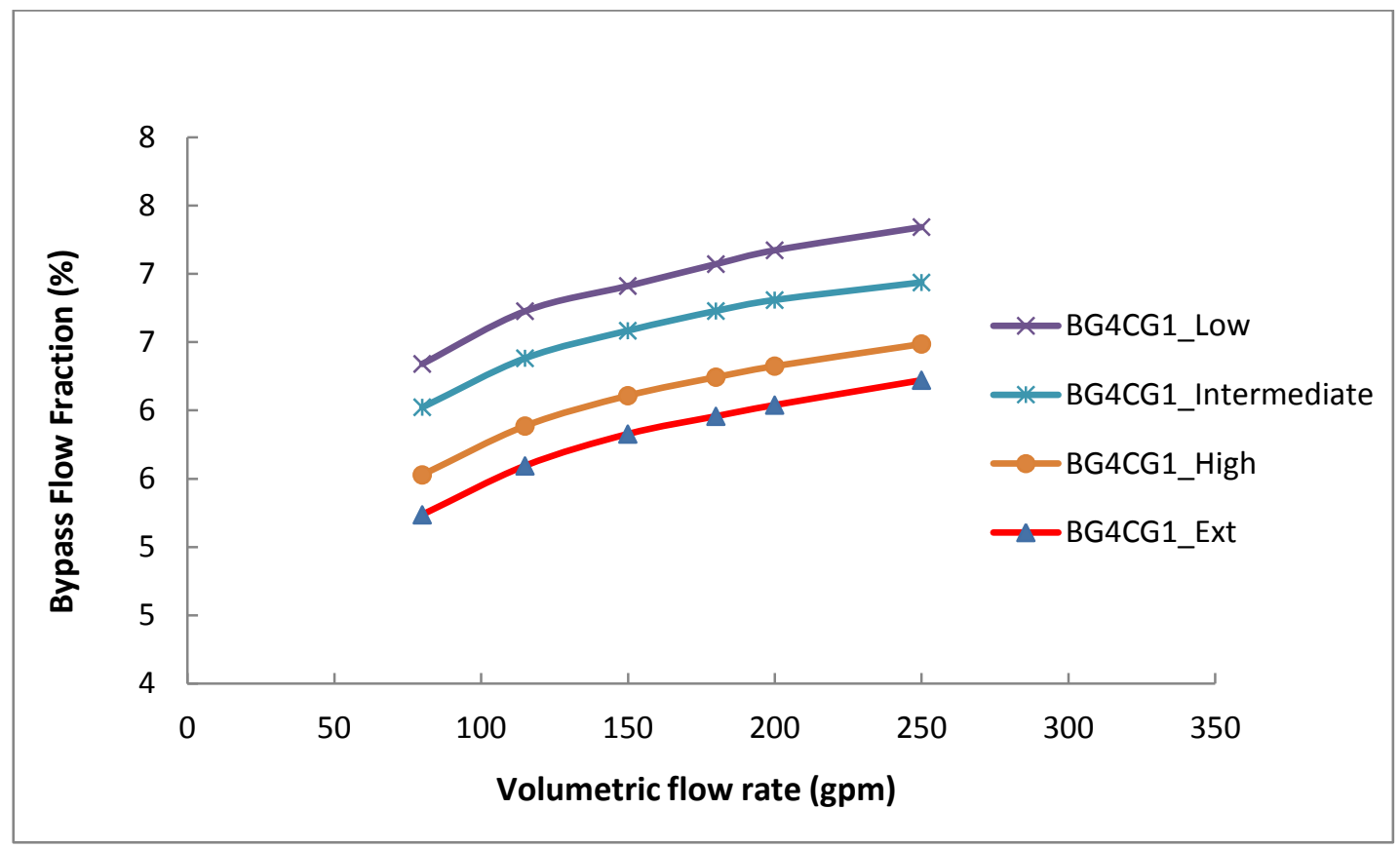

Fig.19 Bypass flow fraction vs. Volumetric flow Rate (BG4CG1)

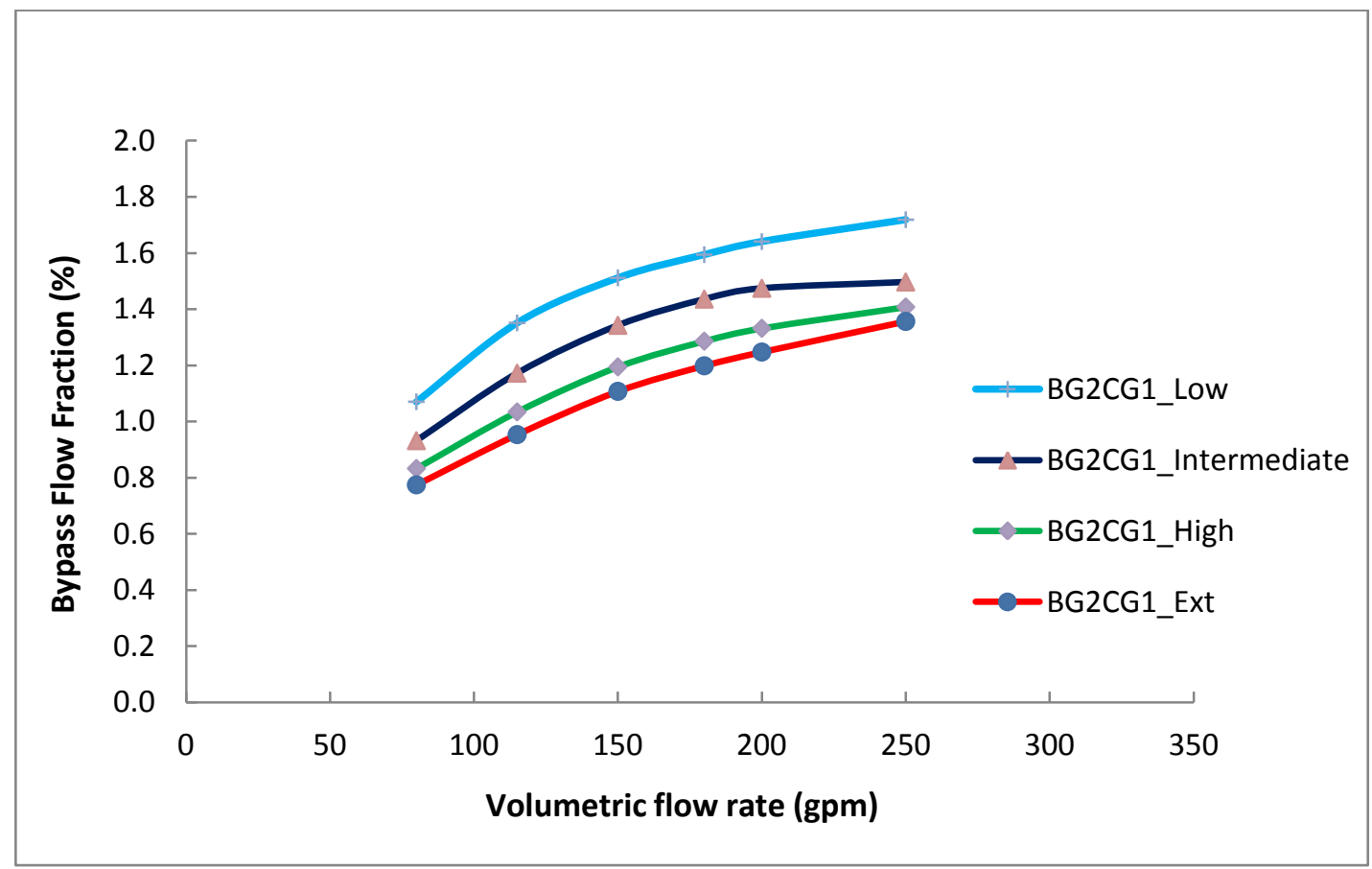

Fig.20 Bypass flow fraction vs. Volumetric flow Rate (BG2CG1) 
The discrepancy regards to the bypass flow fraction ranges from $0.4 \%$ (BG2CG1) to $2 \%$ (BG6CG1). This discrepancy decreases when the bypass flow gap becomes narrower as the total number of cells for the $2 \mathrm{~mm}$ bypass flow gap case is much greater than that of the $6 \mathrm{~mm}$ case. A larger number of cells means that the truncation error is reduced. Clearly, even the coarse polyhedral mesh can meet the requirement since the difference between the coarse and fine mesh is not significant although the model with coarse mesh does overestimate the bypass flow fraction. Another interesting observation is the coupling relationship between the bypass flow fraction and the flow rate becomes weak when the gap is narrow. For the $2 \mathrm{~mm}$ bypass gap case, the slop reduces significantly when the flow rate reaches $250 \mathrm{gpm}$. It is because the resistance within the narrow gap is so large that it is not easy for coolant to flow into the gap even with a higher flow rate.

Extrapolated values were also calculated based on the Richardson Extrapolation Method shown as the red lines in these figures. Considering the fact that finer mesh can reduce the discretization errors only variables $\Phi_{1}$ and $\Phi_{2}$ were selected to calculate the extrapolated value $\Phi_{\text {ext }}^{21}$ using Eq. (33). All three figures show that the discrepancies between the extrapolated value and the solution from the finest mesh are relatively small. The extrapolated solutions can be treated as solutions from the "finest" mesh which is only achievable in theory. However, it is obvious not the exact or analytic solution because there are other sources of errors, such as modeling errors, round off errors and so on. The significance of the extrapolation values is that it provides the criteria to evaluate that how far is the solution obtained from a mesh of certain density 
(fine or coarse) from the solutions based on the "finest" mesh in theory. Fig. 21 combines these three figures together to demonstrate the relative trend for each case. It is clear that the discrepancy is reduced when the bypass flow gap width decreases. In addition, coarser mesh is trending to predict a higher bypass flow fraction. Thus an optimized mesh is required in order for obtaining a relatively accurate solution with respect to the computational cost.

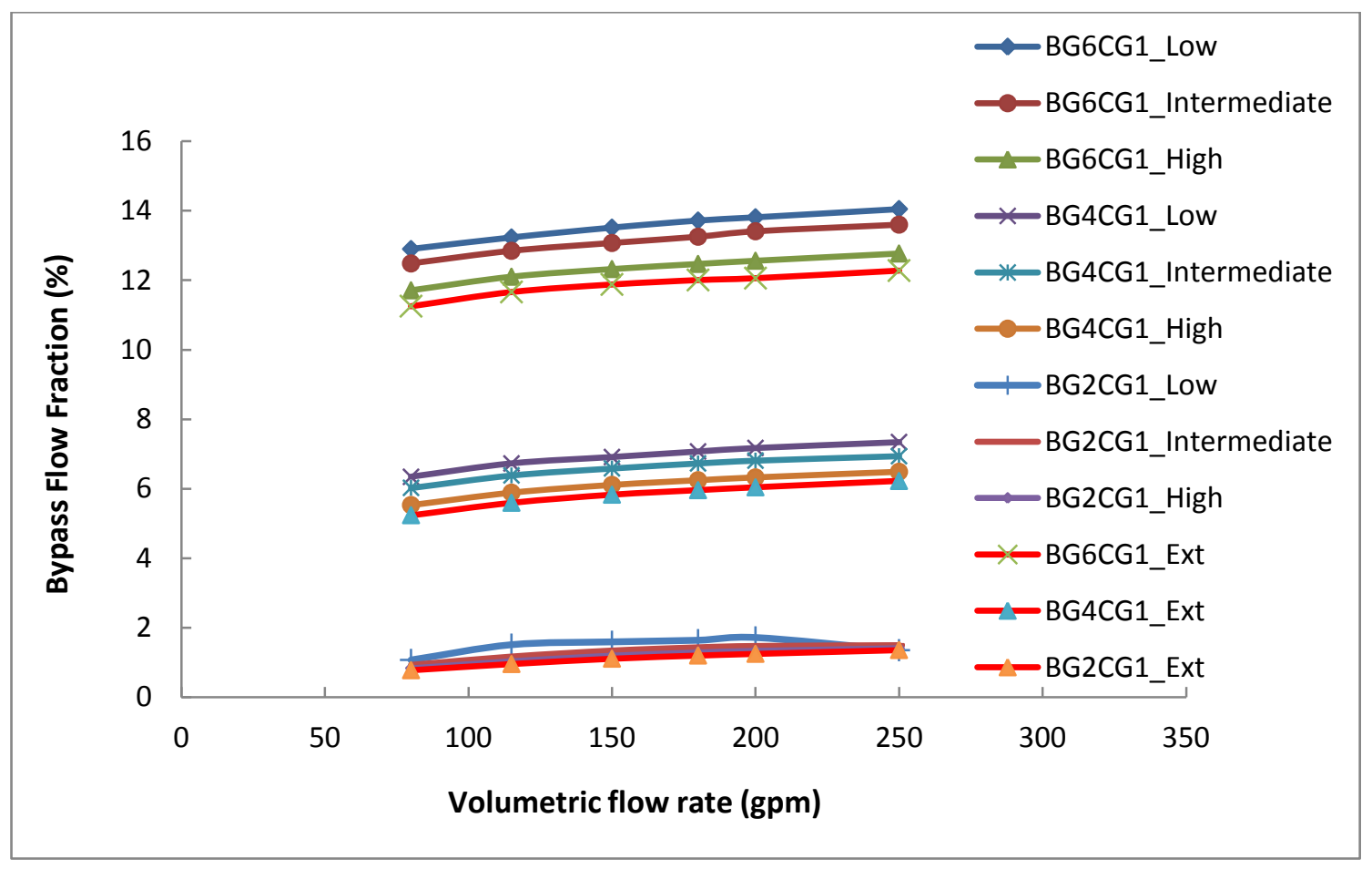

Fig.21 Bypass flow fraction vs. Volumetric flow Rate (All Cases)

To sum up, the mesh with good quality is prerequisite for generating a relatively accurate solution by reducing the discretization errors. To achieve this goal the grid independence study is recommended to be performed to seek the optimized mesh sizes. 
Richardson Extrapolation Method can be employed to calculate the extrapolated values from a set of meshes of different sizes. The discretization errors are quantified by comparing the solutions obtained from the simulation with those extrapolated values. 


\section{RESULTS AND DISCUSSION}

Although the grid independence study indicates that the fine mesh offers a solution with smaller error, a different mesh size matrix was selected to present the results. The total number of cells is larger or equal to the fine mesh case. There is no any meaningful reason for this option but only simply because simulations used to demonstrate the results in this chapter were generated before the grid independence study was performed. It is acceptable to do the analysis in this sequence as long as the corresponding uncertainties are quantified and reported. Uncertainties were calculated based on the algorithm discussed in previous chapter and the results will be discussed in next chapter. Table 4 shows the information of the meshes used for generation of the solutions.

Table 4 Total number of cells for each case investigated

\begin{tabular}{|c|c|}
\hline Case & Number of cells (million) \\
\hline BG6CG1 & 21.2 \\
\hline BG4CG1 & 22.6 \\
\hline BG2CG1 & 40.4 \\
\hline BG6CG0 & 15.3 \\
\hline BG4CG0 & 12.5 \\
\hline BG2CG0 & 14.0 \\
\hline BG6CG1_2* & 24.8 \\
\hline
\end{tabular}

*1_2 means the crossflow gap ranges from $1 \mathrm{~mm}$ to $2 \mathrm{~mm}$ and it is a wedge-shape gap.

\subsection{Effect of the Bypass Flow Gap Width}

In the prismatic Very High Temperature Gas-cooled Nuclear Reactor (VHTR), the dimension of the reflector (graphite) changes as a result of the presence of radiations 
and the thermal expansion and shrinkage. However, it is difficult to determine what the exact gap size present in the reactor because the change is a dynamic process and the gap size differs from reactor by reactor. Thus it is necessary to analyze the coolant distribution with different gap sizes present in the reactor. In present work, three sizes of the bypass flow gap were studied, $2 \mathrm{~mm}, 4 \mathrm{~mm}$, and $6 \mathrm{~mm}$. The bypass flow gap is assumed to be uniform through the reactor and the crossflow gap effect was not considered in this section. Fig. 22 presents the influence of the bypass flow gap width to the bypass flow fraction for different Reynolds numbers.

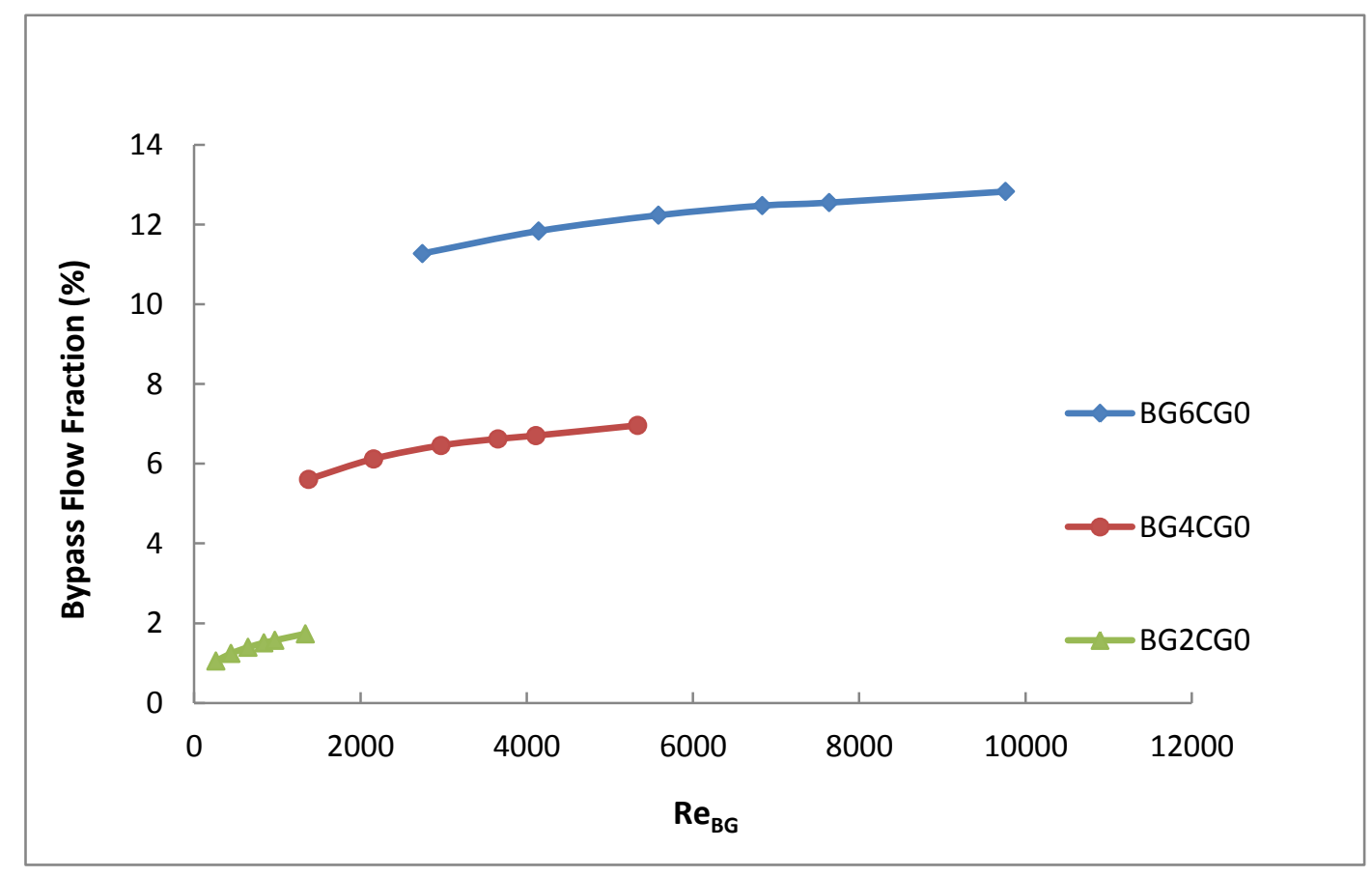

Fig.22 Bypass flow fraction vs. $\mathrm{Re}_{\mathrm{BG}}$ for varied bypass flow gap widths

It is seen that the bypass flow fraction is proportional to the gap width and the Reynolds number. The fraction ranges from $11.3 \%$ to $12.8 \%$ for the widest gap and from 
$1.1 \%$ to $1.7 \%$ for the narrowest one. This phenomenon can be explained that the coolant will confront more resistance from the narrower gap so more fluid will flow into places in which the resistance is smaller. Those places refer to the coolant channels where the coolant efficiency is much higher. However, this does not guaranty that there will be hot spots appearing when the gap is large because coolant is still able to cool down the graphite around the gap region, which still can remove certain amount of heat. Power distribution involving neutronic calculations is required before drawing any conclusion.

Another point which needs to be looked into is the Reynolds number in the bypass flow gap. Approximately, flow becomes turbulent when the Reynolds number is greater than 1,300 for the flow within narrow parallel plates. Thus, the flow is mainly laminar flow for the $2 \mathrm{~mm}$ bypass flow gap case. In contrast, flows in the $4 \mathrm{~mm}$ and $6 \mathrm{~mm}$ gap are turbulent. This is mainly because the space for the flow is so narrow for the $2 \mathrm{~mm}$ gap that the chance for any eddies, swirls or significant fluctuations become small. These fluctuations would be dampened by the very narrow gap if there has been any tendency for them to be formed. But it doesn't mean the flow cannot be turbulent but just indicates that the turbulence may not exist in the case studied in the present work.

\subsection{Effect of the Crossflow Gap}

As mentioned earlier, bypass flow fraction is a key factor during the VHTR design process. A high bypass flow fraction may be a threat to the nuclear reactor safety while an appropriate value of the fraction may significantly cool the near-gap region (Sato, H., Johnson, R., and Schultz, R. 2010). However, the presence of the crossflow 
gap may also impact the coolant distribution in the core. The mechanism is that the coolant will be redistributed when passing through the crossflow gap because of the pressure difference in the bypass flow gap and the coolant channels. In order for a better understanding of the crossflow gap discussed in the present work, a schematic figure is presented below. Fig. 23 (a) is for the case of no crossflow gap. Fig. 23 (b) represents the $1 \mathrm{~mm}$ uniform crossflow gap case and (c) illustrates the condition when the $1^{\text {st }}$ layer of blocks is titled so a wedge-shape crossflow gap is formed. The minimum width is $1 \mathrm{~mm}$ and the maximum is $2 \mathrm{~mm}$.

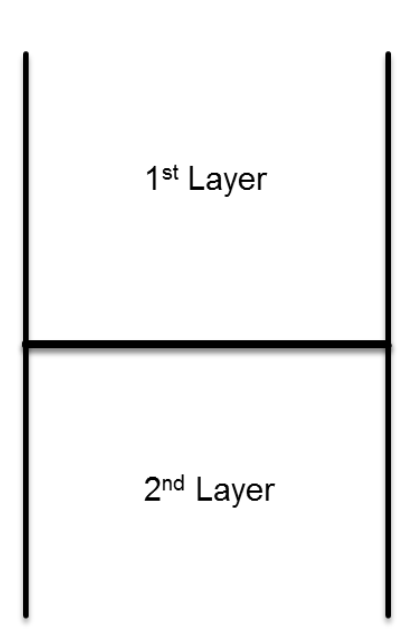

(a) No gap

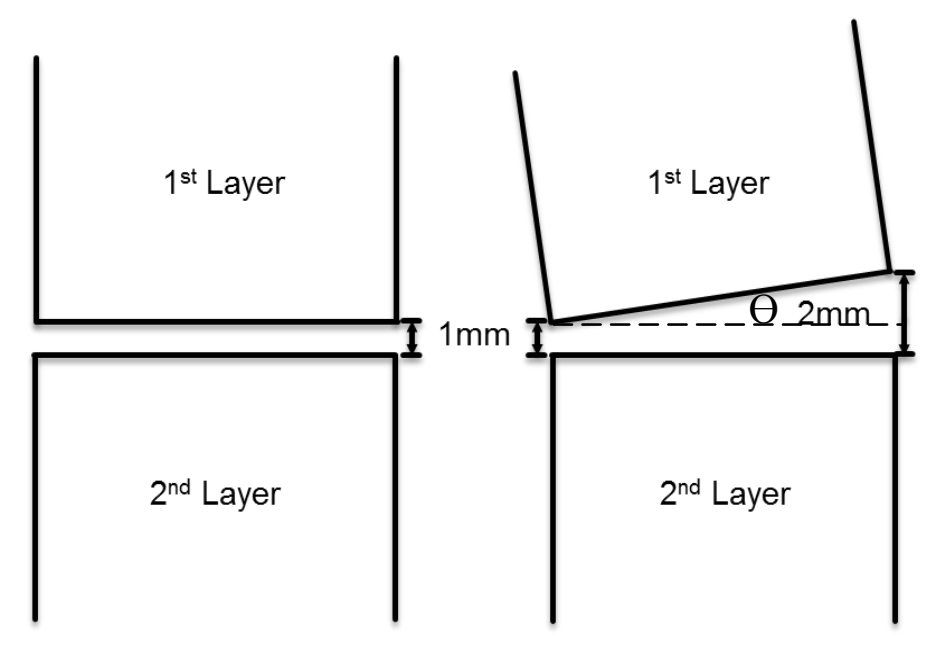

(b) $1 \mathrm{~mm}$ uniform gap

(c) 1-2mm wedge-shaped gap

Fig.23 Sketch of crossflow gap shapes

For the wedge-shape gap the inclination angle, $\Theta$ shown in the Fig. 23 (c), is only $0.2^{\circ}$ which is a very small angle. However, it is this small angle that changes the coolant flow path and may bring significant effect on the pressure and velocity distributions. Fig. 24 demonstrates the relationship between the bypass flow fraction and the Reynolds 
number in the bypass flow gap for three cases, uniform, wedge-shape and absence of crossflow gap. Please note if there is no specific explanation, the default bypass flow gap is $6 \mathrm{~mm}$.

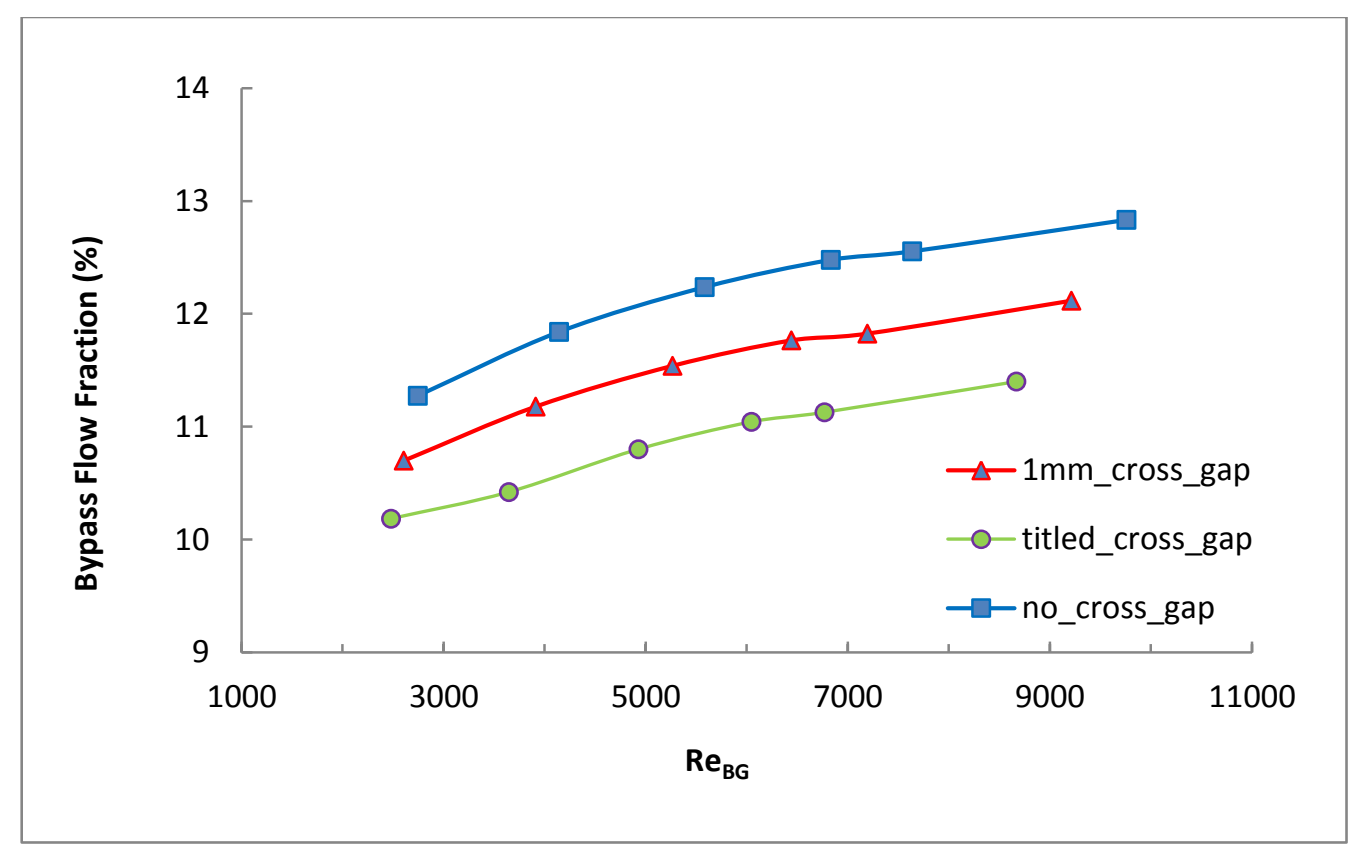

Fig.24 Bypass flow fraction vs. $\operatorname{Re}_{\mathrm{BG}}$

Here Reynolds number has its usual meaning which is defined in Eq. (38).

$$
R_{e B G}=\frac{\rho * v_{b} * D_{h}}{\mu}
$$

where $\mathrm{v}_{\mathrm{b}}$ is the average velocity in the bypass flow gap and it is calculated based on the mass flow rate and the cross area of the bypass gap. $D_{h}$ is the hydraulic diameter of the bypass gap and other symbols have their usual meanings.

The overall bypass flow fraction is about $12 \%$ which is close to the real reactor operating conditions. A higher Reynolds number produces a larger bypass flow fraction. 
For the same Reynolds number, the case with a titled crossflow gap has the largest bypass flow fraction while the fraction for the case without crossflow gap is the smallest. This can be explained by the fact that the flow will be redistributed when it passes by a crossflow gap. The pressure in the bypass gap is higher than that in the coolant channel so some portions of the flow originally from bypass gap may prefer to flow into the coolant channel where the resistance is much lower. For the titled and uniform crossflow gap cases, titled one provides more room for the flow to be redistributed in the crossflow gap region, which results in a lower bypass flow fraction.

In order to evaluate the pressure loss in the system in a dimensionless way, a parameter named pressure loss factor $\xi$ is defined as:

$$
\xi=\frac{\Delta P}{\frac{1}{2} \rho v_{b}^{2}}
$$

where $\Delta \mathrm{P}$ is the summation of the major pressure loss due to friction and minor pressure loss due to flow area changes; $\rho$ is the density of the fluid (water for the present study); $v b$ is the same as that in Eq. (38). The pressure loss factor as a function of Re_bypass is plotted in Fig. 25. 


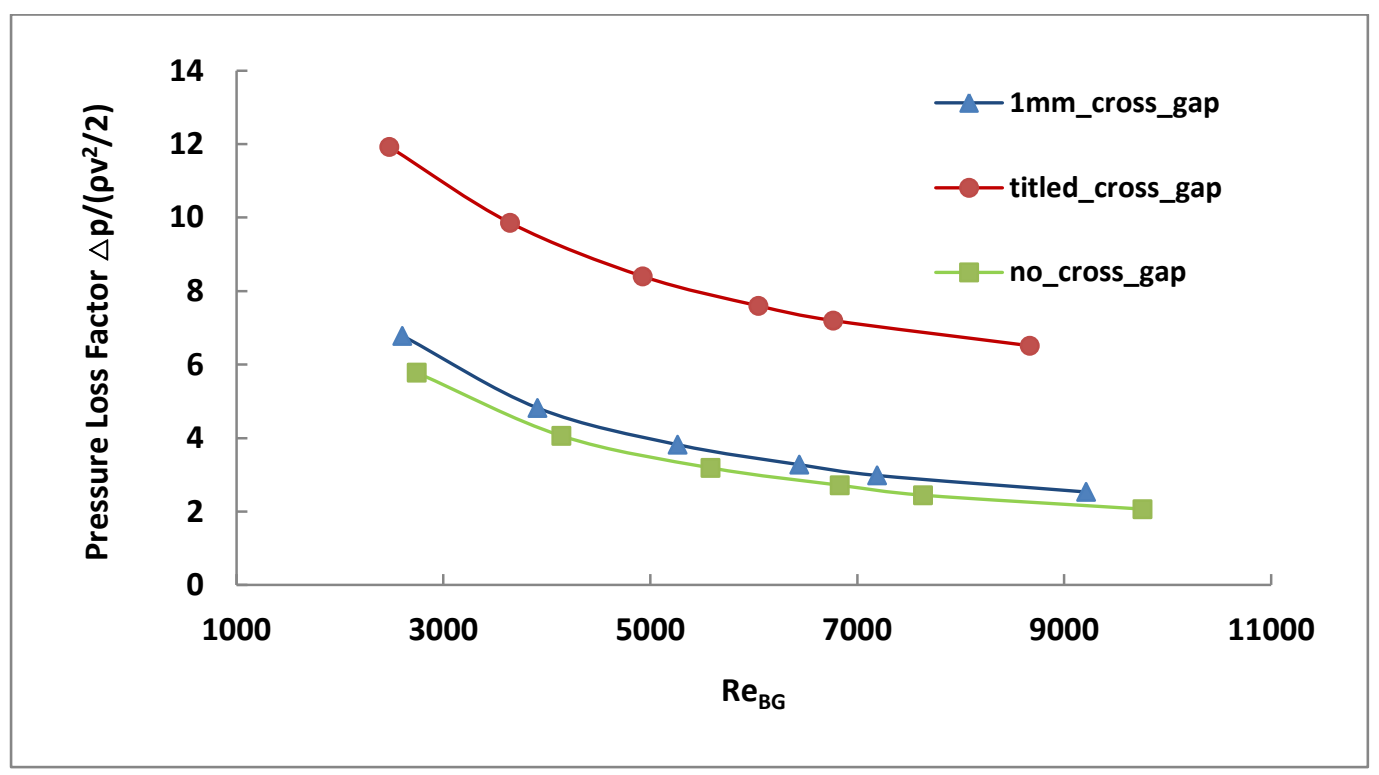

Fig.25 Pressure loss factor vs. $\mathrm{Re}_{\mathrm{BG}}$

Although the three cases share the same trend again but obviously the model with a titled crossflow gap has a significantly higher pressure drop. The reason may be that flow is redistributed in the crossflow gap region, which produces a large number of recirculations. These eddies increase the energy dissipation and large pressure drop can be expected. Also, it is highly possible that certain amount of low will hit the wall between coolant channels instead of directly flow into those holes due to inclined flow paths, which also can increase the pressure loss.

The velocity field were computed though the middle plane of the crossflow gap for both the uniform gap case and the wedge-shape gap case with a volumetric flow rate of $180 \mathrm{gpm}$ (11.36 in kg/s approximately). Fig. 26 and Fig. 27 present the tangential velocity field. The normal vector of the middle plane is parallel to the flow direction in 
the positive $\mathrm{z}$ direction. In Fig. 27, the $1 \mathrm{~mm}$ crossflow gap side (lower side) is located at the lower right corner while the $2 \mathrm{~mm}$ one (higher side) is at the upper left corner.

It is noticeable that no matter the block is titled or not there is a strong secondary flow moving from the bypass flow gap towards coolant channel regions. This horizontal flow is very important as it will increase the mass flow rate in the coolant channels.

Those coolant holes far from the bypass flow gap will receive less increment compared to those in the regions close to the gap, which may result in an uneven distribution of the coolant. It is possible that this asymmetry would bring in physical vibration of the block itself and also influence the temperature distribution in the fuel region. The differences in these two figures lie in that the horizontal flow is able to flow faster and further towards coolant channels for the wedge-shape crossflow gap.

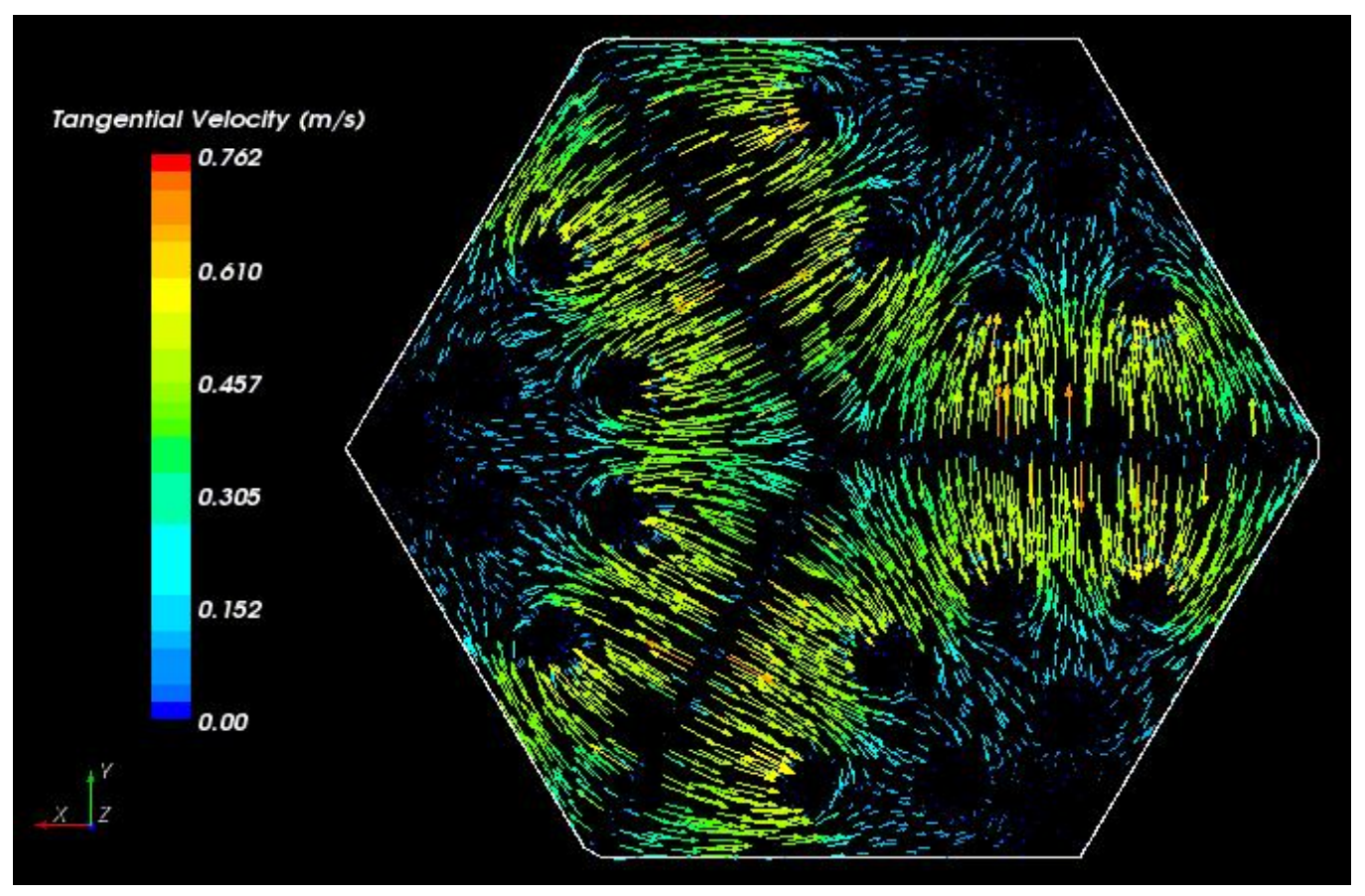

Fig. 26 Velocity field for the uniform gap case 


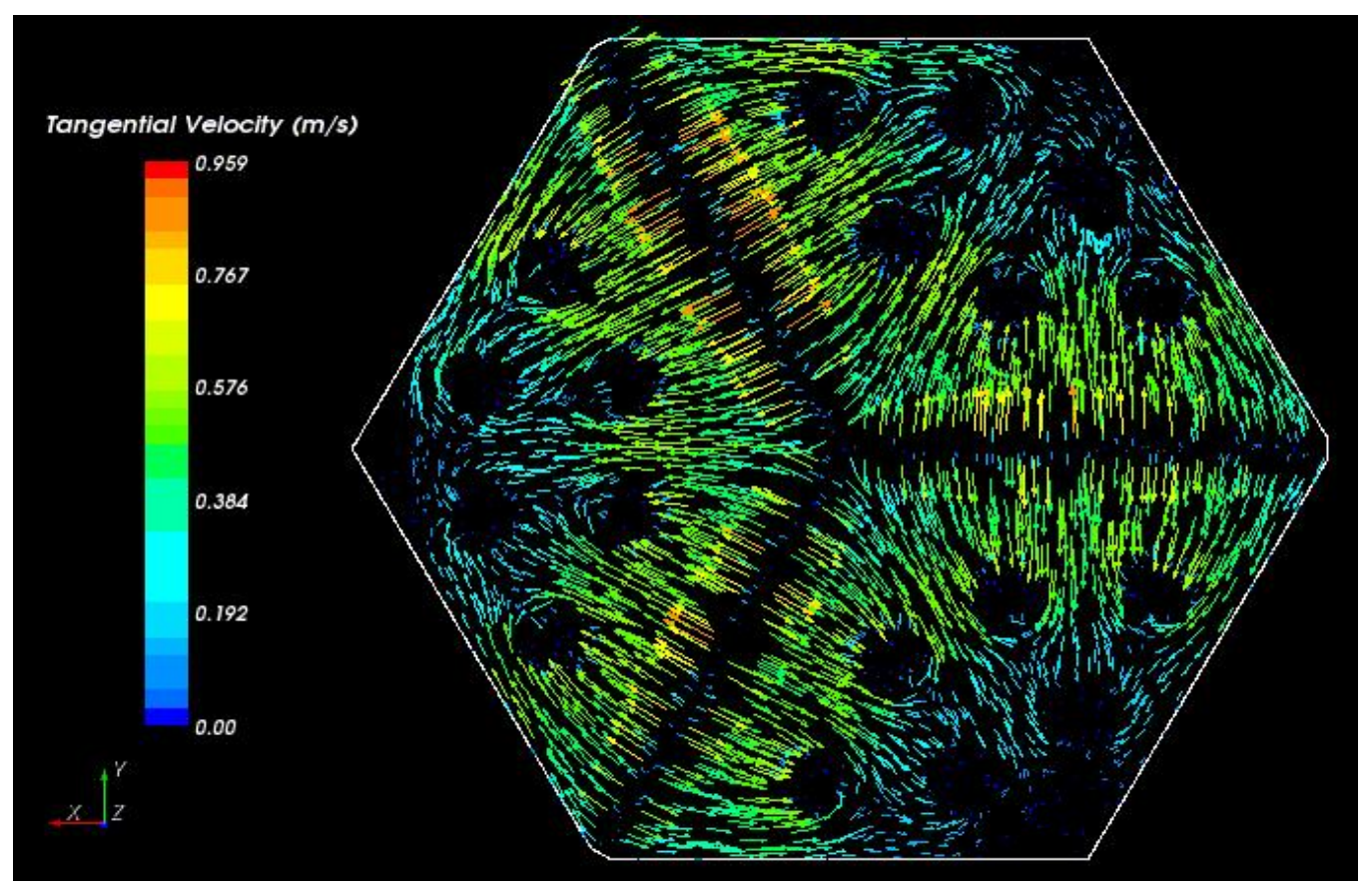

Fig.27 Velocity field for the wedge-shape gap case

To verify the statements above, it is necessary to investigate the influence to the mass flow rate distribution from the crossflow gap. To begin with, mass flow rates are computed for each single coolant channel and bypass flow gap on both Plane A and Plane B illustrated in Fig. 28. These two planes are located at the $1 \mathrm{~mm}$ higher of the crossflow gap while the other one is at $1 \mathrm{~mm}$ below the gap respectively. 


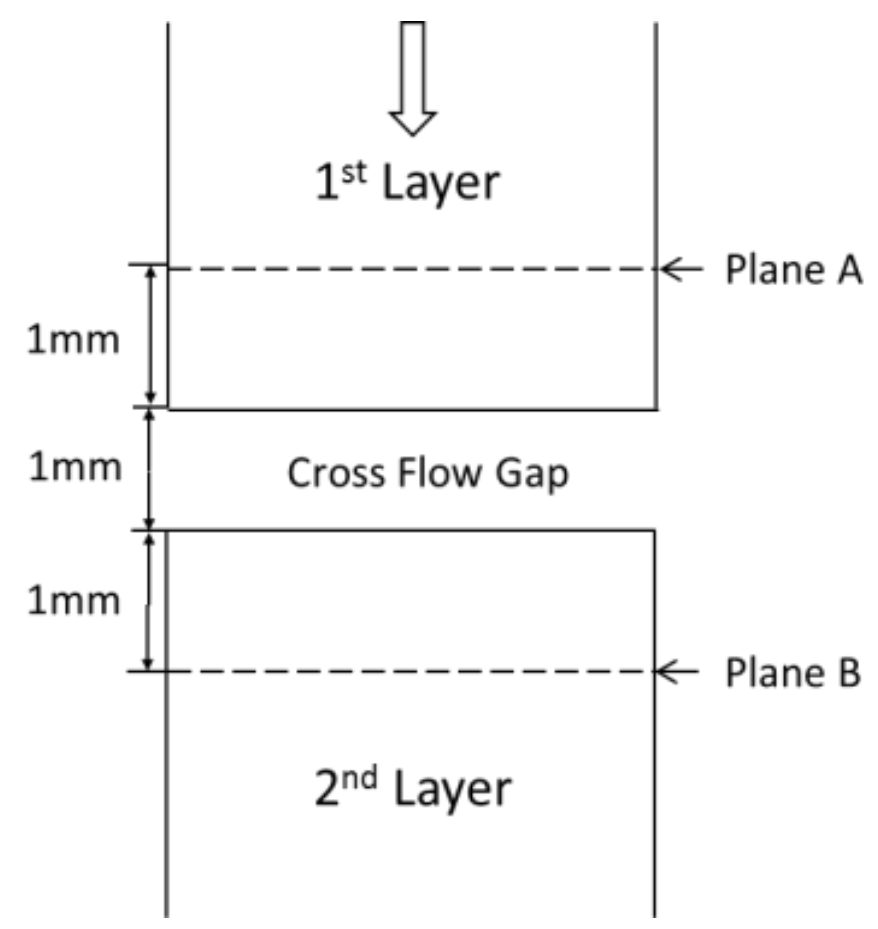

Fig. 28 Sketch of planes used for calculation

Although for a uniform crossflow gap the mass flow rate is expected to be evenly distributed though the interested planes, the computational results show that this may not be the case. Fig. 29 presents the mass flow rate in percentage with respect to the total flow rate for both coolant channels and bypass flow gap region. The values in the peripheral region are slightly higher than those in the inner part which can be explained that bypass flow gap "steal" a small portion of coolant which should originally flow into those coolant holes near to the bypass flow gap. Overall, the mass flow rate in each coolant hole is symmetrical along the bypass gap. 


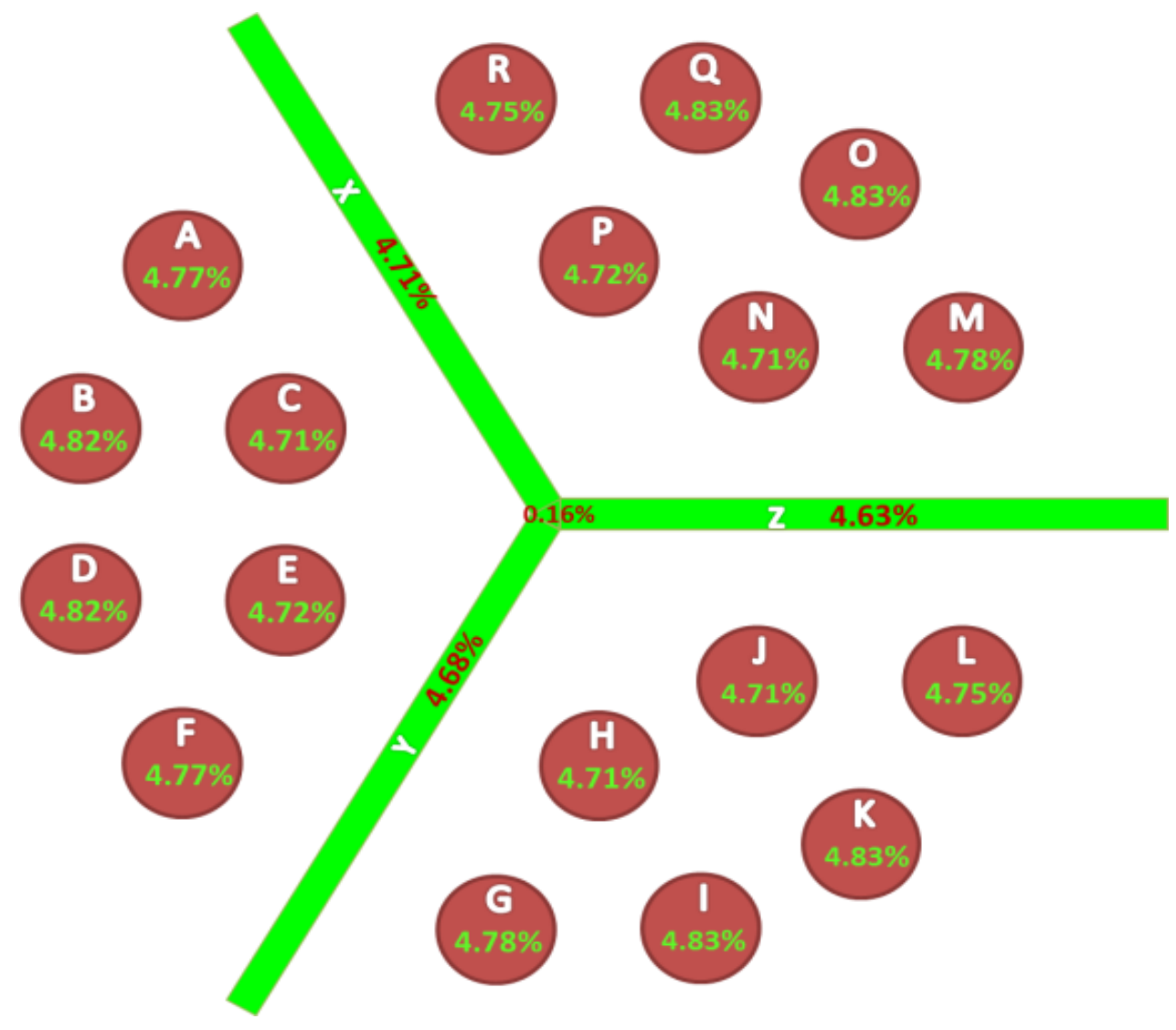

Fig.29 Mass flow rate on Plane A for the uniform gap case

After the flow pass through the crossflow gap, the flow will be redistributed.

Mass flow rates on Plane B are computed and Fig. 30 indicates the change between values on Plane A and Plane B in percentage with respect to mass flow rates on Plane A. 


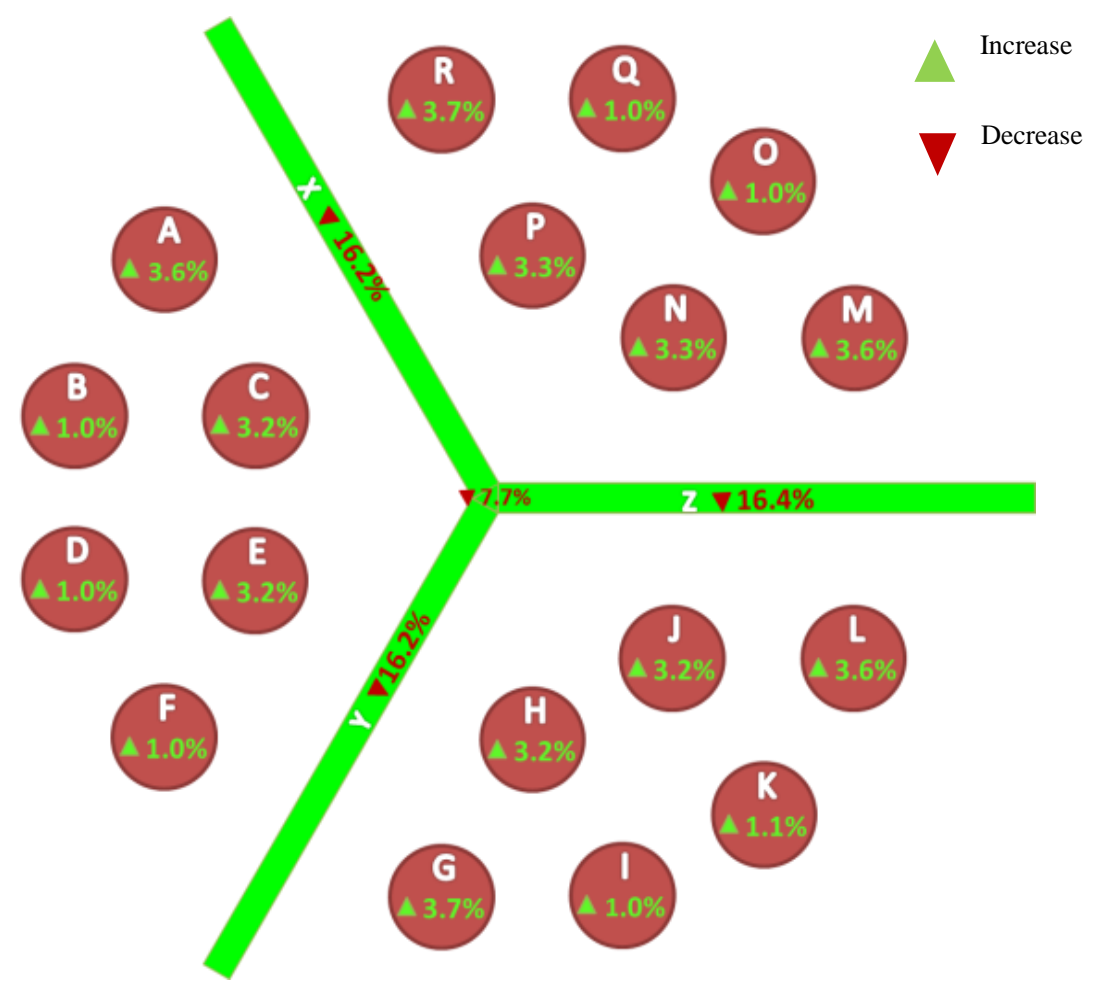

Fig.30Mass flow rate change for the uniform gap case

The distribution of mass flow rate change shows a good symmetry along the bypass flow gap. As expected above, more coolants flow into those coolant channels near to the gap. Meanwhile, flow in the bypass flow gap decreases by about $16 \%$ for the three partial sections and $7.7 \%$ for the small triangular region in the middle. It is hard to draw any conclusions about whether the drop in the bypass flow gap is good or not because this may reduce the cooling effect for those fuel rods near to the bypass flow gap although the cooling in coolant holes is more efficient. Hence, more works involved in heat transfer need to be done. 
Fig. 31 demonstrates the mass flow rate change for the titled block. The mass flow rate distribution is in accordance with the velocity field with more significant increase at the upper left corner. It can be observed that there is an approximately $23 \%$ drop for the coolant in the bypass flow gap and 4-5\% increase in the coolant channels while the values for the upper left corner are $-28.8 \%$ and $+6.1 \%$ respectively.

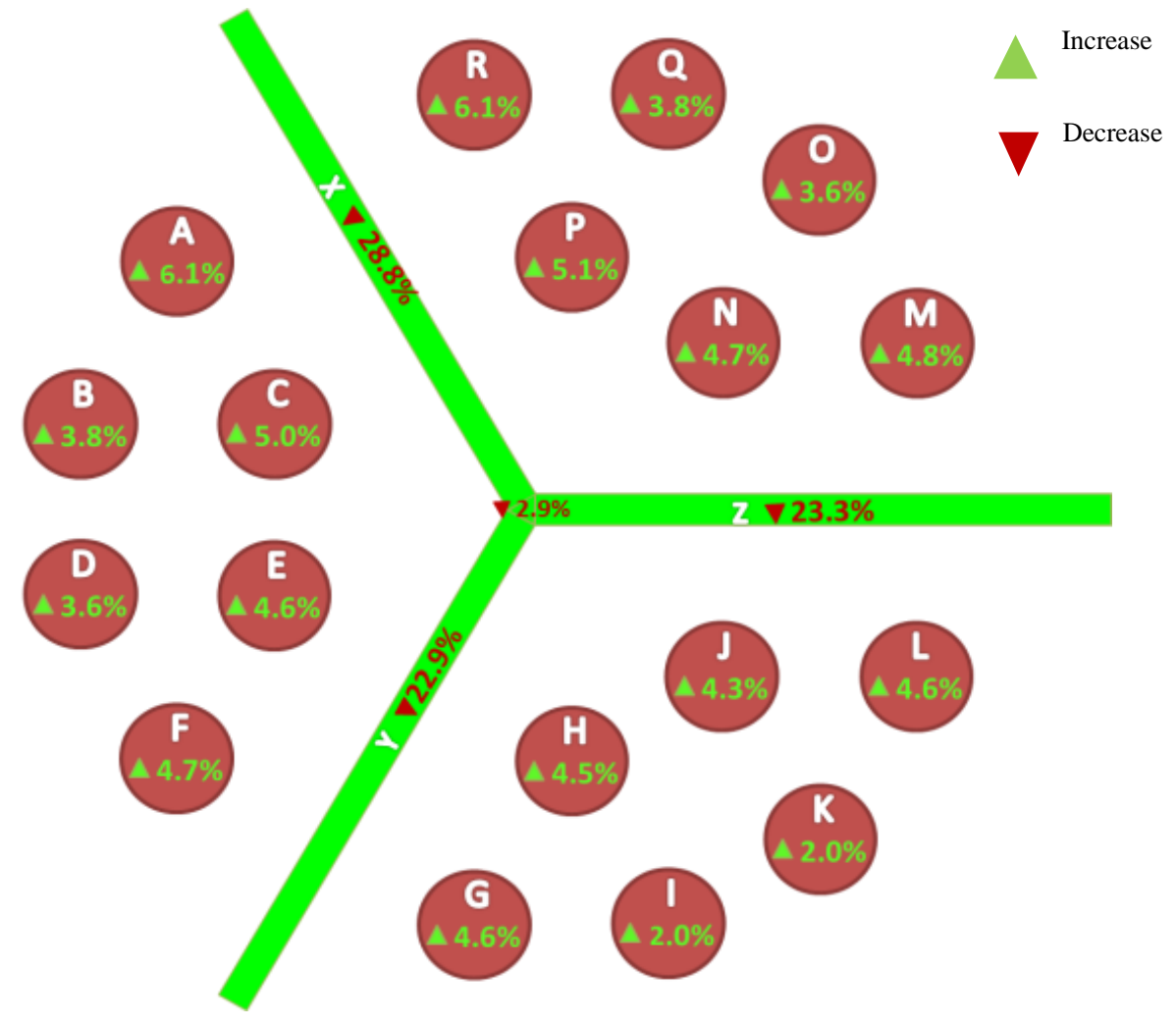

Fig.31 Mass flow rate change for the wedge-shape gap case

Another important concern besides the mass flow rate distribution is the pressure field. Fig. 32 presents the pressure distribution for the wedge-shape crossflow gap 
computed on the same plane shown in Fig. 26. It is seen that there is about $500 \mathrm{~Pa}$ pressure difference between the bypass flow gap and coolant holes, which produces the secondary flow observed in Fig. 27.

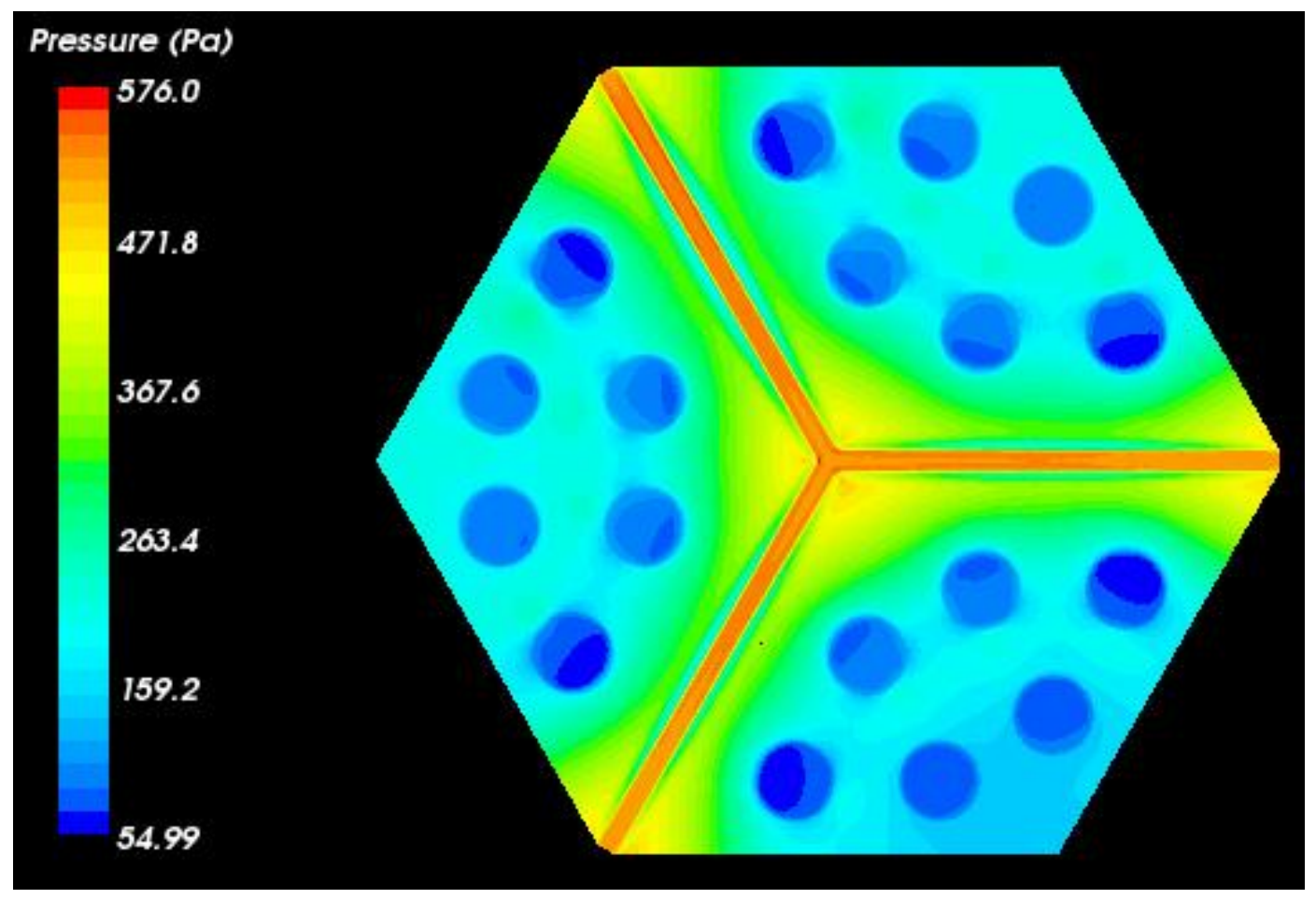

Fig.32 Pressure distribution within the crossflow gap

The static pressure distributions in a coolant channel (\#H in Fig. 31) and in the bypass flow gap are shown in Fig. 33. The measuring point for the bypass flow gap is located in the middle of the section \#Y in Fig. 31. The hydraulic pressure drop due the gravity is ignored and the pressure at the outlet boundary is taken as zero. It is observed that there is significant pressure drop at the inlet of the coolant channel and the bypass 
flow gap due to area reduction. Also pressure decreases by $180 \mathrm{~Pa}$ after the flow pass through the crossflow gap.

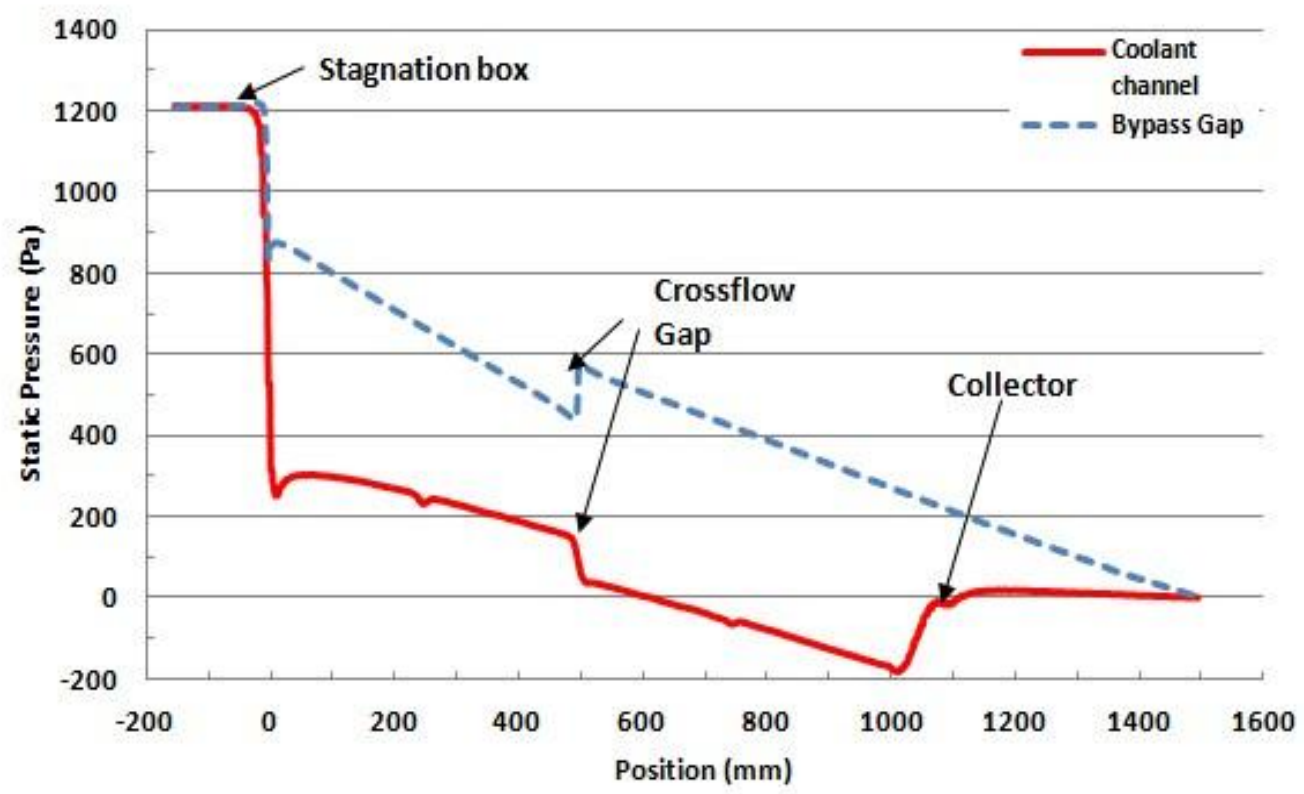

Fig.33 Static pressure distribution

Due to the fact that the pressure drop is related to the flow entrance length in the coolant channel, it is of interest to investigate how the flow develops in the coolant channel. Six planes A-F shown in Fig. 34 are taken at the first layer of the facility. Plane $\mathrm{A}$ is located at $0 \mathrm{~mm}$ in the positive $\mathrm{z}$ direction and $\mathrm{F}$ is at $450 \mathrm{~mm}$. It should be clear that the bottom of the stagnation box or the inlet of the coolant channels is at $\mathrm{z}=-4 \mathrm{~mm}$ and the height of the first layer is $500 \mathrm{~mm}$. 


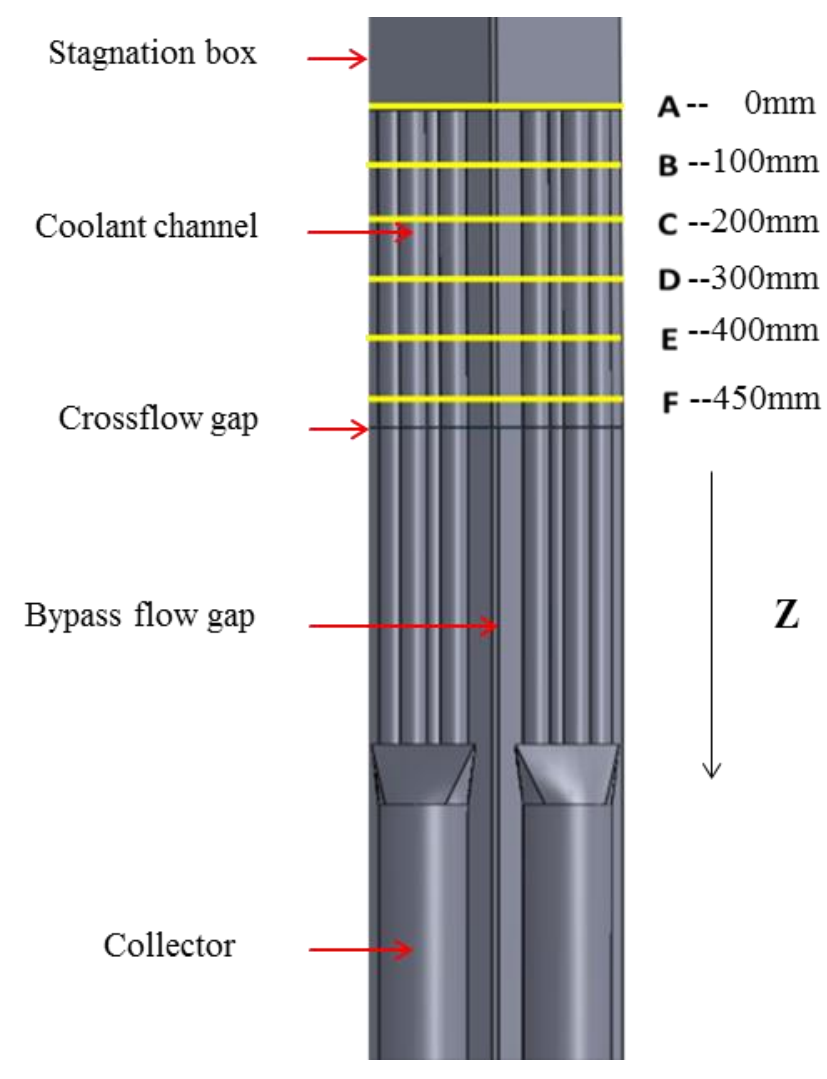

Fig.34 Planes used for entrance length study

The velocity profile is computed in the coolant \#A shown in Fig. 31 for the wedge-shape crossflow gap case with a Reynolds number of 28076 in coolant holes. Fig. 35 presents the result from which it is seen the flow is turbulent and is still developing at least at Plane $E(z=400 \mathrm{~mm})$ as the shape is still changing. When it comes to $\mathrm{z}=450$ $\mathrm{mm}$, the change of the profile is very small which can be observed in the zoom-in circle (a) and (b). However, the change is not small enough to be neglected. That is, the turbulent flow in the coolant hole \#A in the first layer of the block is not fully developed at least at $\mathrm{z}=450 \mathrm{~mm}$ although it may be close. If a widely used empirical relation 
shown in Eq. (30) is used to determine the entrance length of the turbulent flow, the length will be about $616 \mathrm{~mm}$.

$$
L_{\text {turbulent }}=4.4 R_{e}{ }^{1 / 6} D_{h}
$$

where $\mathrm{L}$ is the entrance length, $\mathrm{Re}$ is the Reynolds number and $\mathrm{Dh}$ is the hydraulic diameter. For the coolant channel in present study, $\mathrm{D}_{\mathrm{h}}$ is the diameter of the coolant hole directly which is equal to $25.4 \mathrm{~mm}$. Fig. 36 shows another scalar scene of the velocity profile in coolant channel \#A (top) and \#F (bottom).

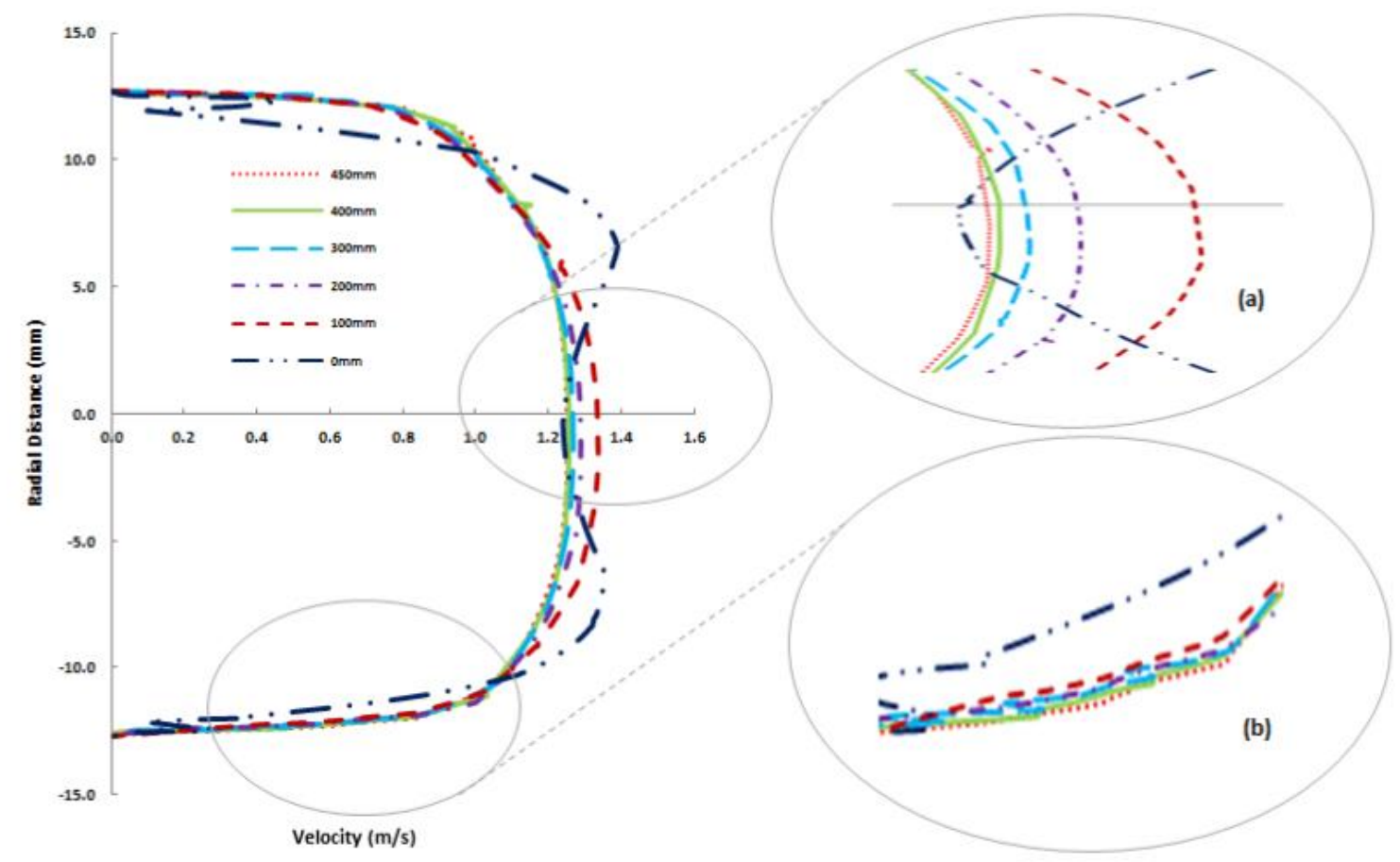

Fig.35 Velocity profile in a coolant channel 


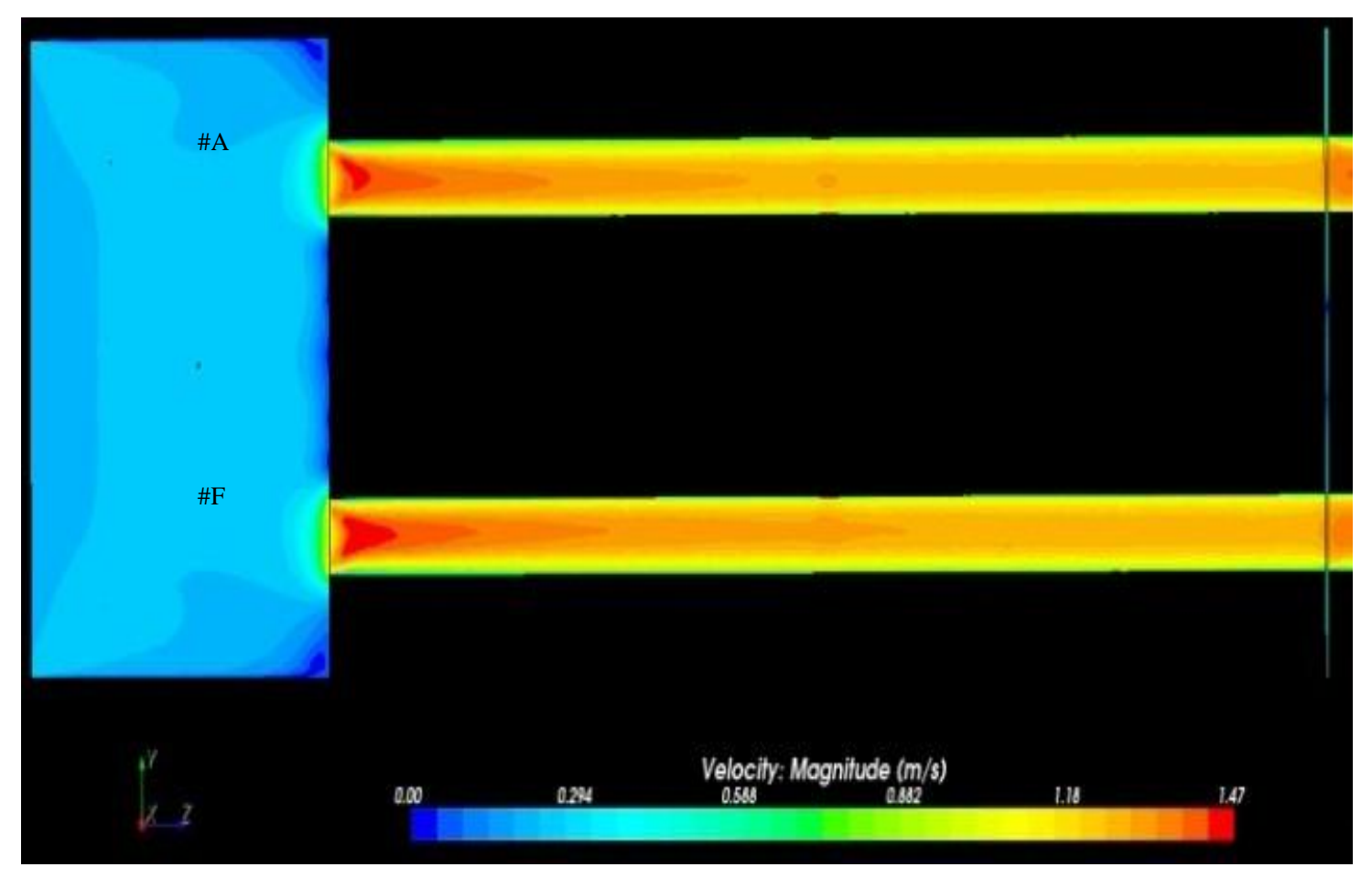

Fig.36 Velocity Profile in coolant channel \#A and \#F 


\section{ERROR ANALYSIS}

Although the application of CFD has been widely used as an engineering tool for its cost saving and enhancement of understating the flow details, how confident the CFD results are is addressed because it would be a huge waste of the money and time if those colorful pictures are meaningless and untruthful. Of course verifying the computational results against the experimental data is a way to quantify these errors but it is necessary to assess the computational errors and uncertainties during the process of CFD modeling itself. According to the guides from AIAA (AIAA, 1998) the error is defined as a recognizable deficiency in a CFD model that is not caused by lack of knowledge. The uncertainty is a potential deficiency in a CFD model that is caused by lack of knowledge (AIAA, 1998). Although there is a difference between them, usually uncertainty analysis is called "propagation of error". The true results of a given problem may not be known but the results could lie in a range with certain uncertain in which results could be trusted. Table 5 provides a classification of sources of errors (NASA, 2012). 
Table 5 Sources of errors in CFD modeling

\begin{tabular}{|l|l|}
\hline & Physical modeling error \\
& Geometry modeling error \\
Acknowledged Error & Round-off error \\
& Iterative convergence error \\
& Discretization error \\
\hline Unacknowledged Error & Computer programming error \\
& Usage error \\
\hline
\end{tabular}

Since the unacknowledged error is negligible compared to the acknowledged one and also it is not accessible usually, acknowledged error is what will be focused on and investigated. More specifically, the iterative convergence error and the discretization error will be quantitatively discussed. The physical modeling error refers to the error generated when a real problem is represented by a mathematical model. For example, the turbulence models widely used in the CFD applications are necessary approximations when solving for the Navier-Stokes equations for the turbulent flow. Error from this modeling process is hard to be quantified except comparing the results against the experimental data. The geometry modeling error is treated negligible because four digits were kept for the dimension of the CFD model built in the SolidWorks in present work. At least three digits were kept for all the calculations involved in the present work and so the round-off error can also be neglected. 
Iterative convergence error is generated when the iteration is terminated in the condition that certain convergence criteria set before the iteration starts is satisfied. For example, it is recommended that at least three or four orders of magnitude of the residuals decrease to achieve the iterative convergence. However, this doesn't mean that the orders dropped smaller than three or four indicate a non-converge condition. The convergence can be evaluated by monitoring if the residuals and the solution of some interested variables are constant or their average momentum doesn't change significantly. In present work, the stopping criteria was set to be 1,000 iterations considering the accuracy and the computational cost. Obviously there were errors involved during this approximating process. For illustrative purpose, Fig. 37 presents the plot of the residuals for the BG4CG0 case with a volumetric flow rate of $80 \mathrm{gpm}$. It is seen that the residuals drop by an order of four and become constant after 700 iterations. Furthermore, bypass mass flow rate was selected as the target variable to investigate the convergence condition of the simulation. The solution as a function of the number of iterations is shown in Fig. 38 and a zoom-in view is followed by Fig. 39 to make sure that any fluctuations were not hidden due to the large range of change of the solution at the beginning of the iteration. It can be found that the convergence had been achieved as the change of the solution is negligible and the residuals are constant as well. 


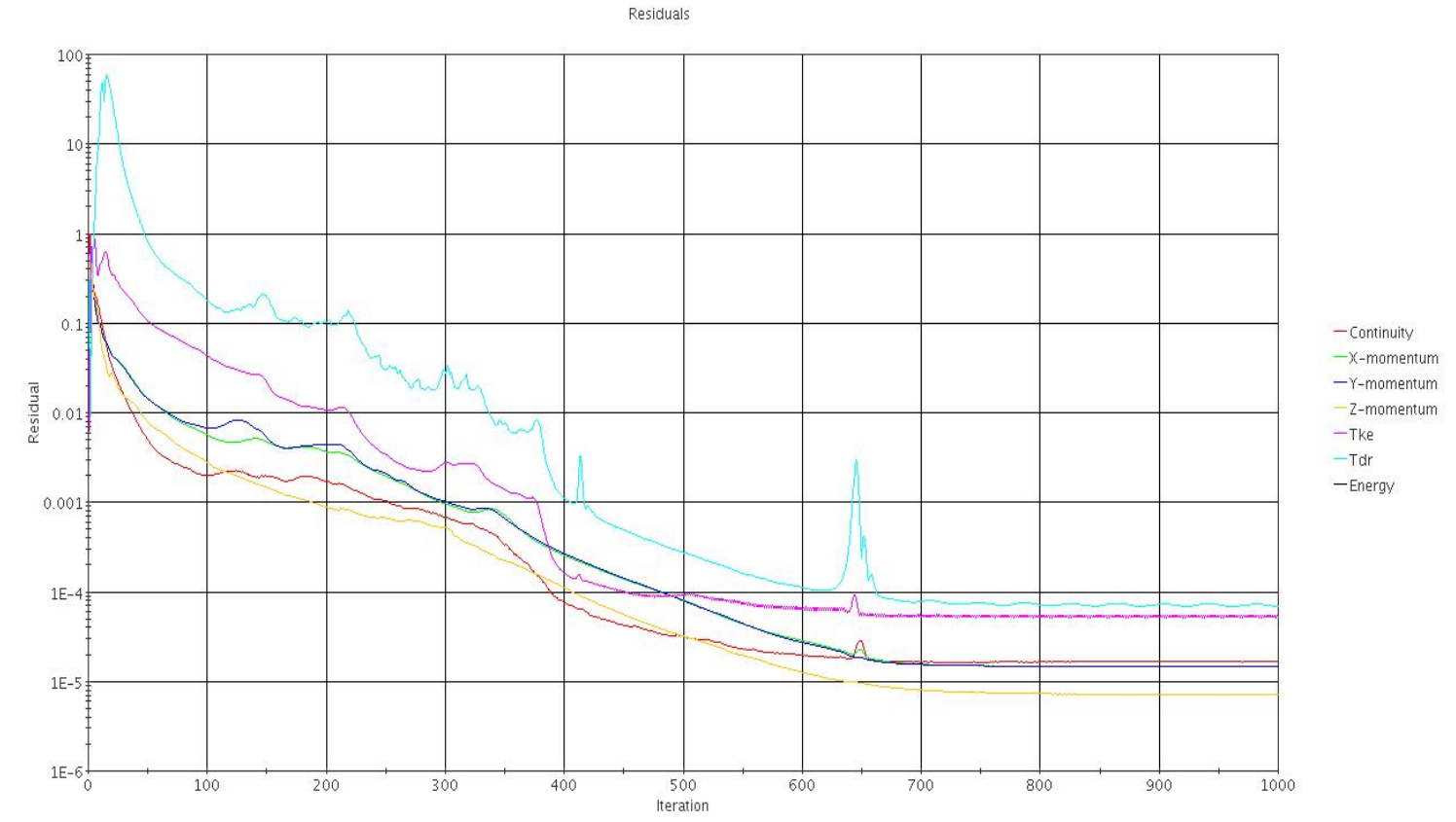

Fig.37 Residuals for the BG4CG0 case with a flow rate of $80 \mathrm{gpm}$

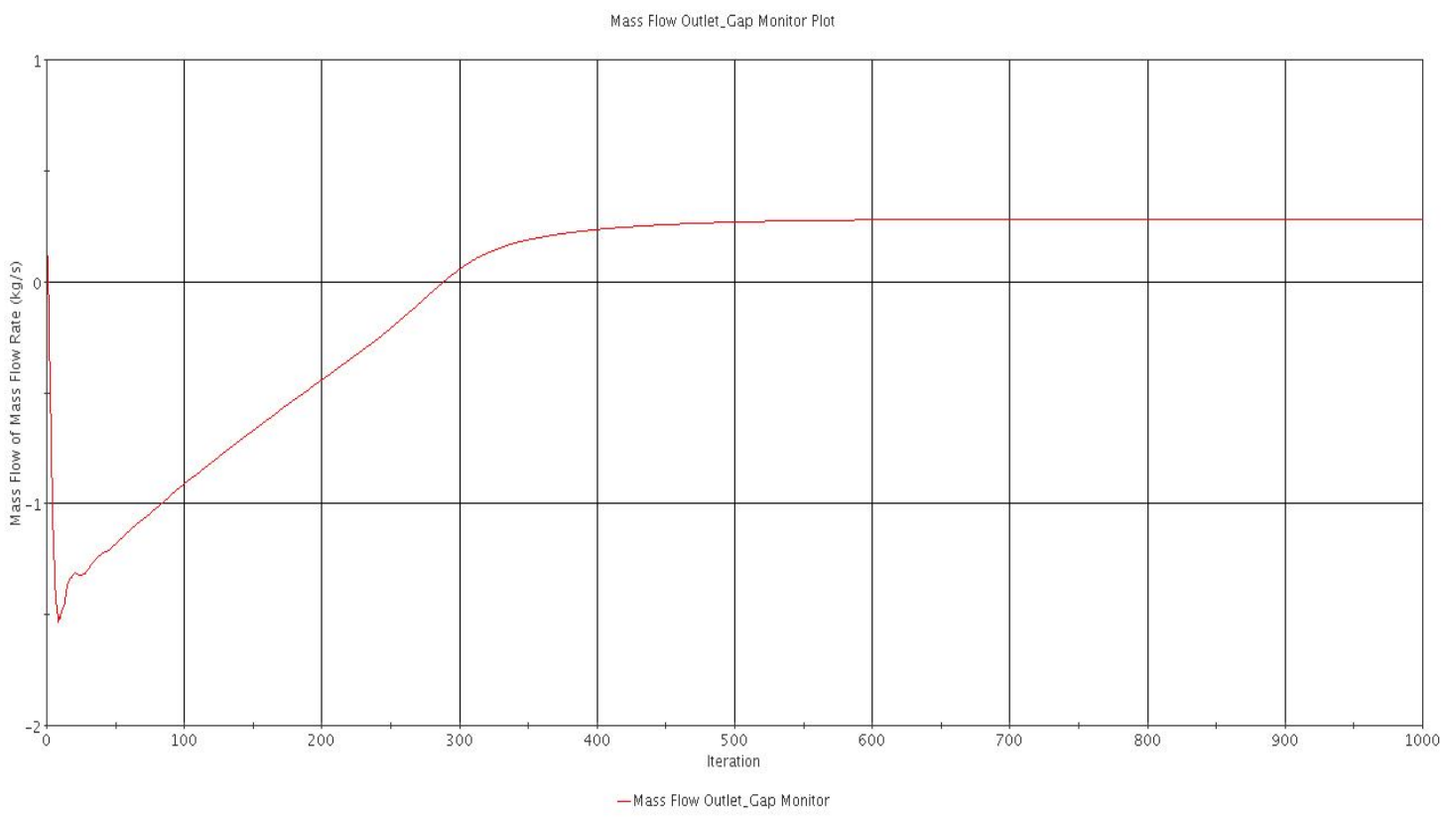

Fig.38 Mass flow rate of the bypass flow at outlet as a

function of the number of iterations 
Mass Flow Outlet_Gap Monitor Plot

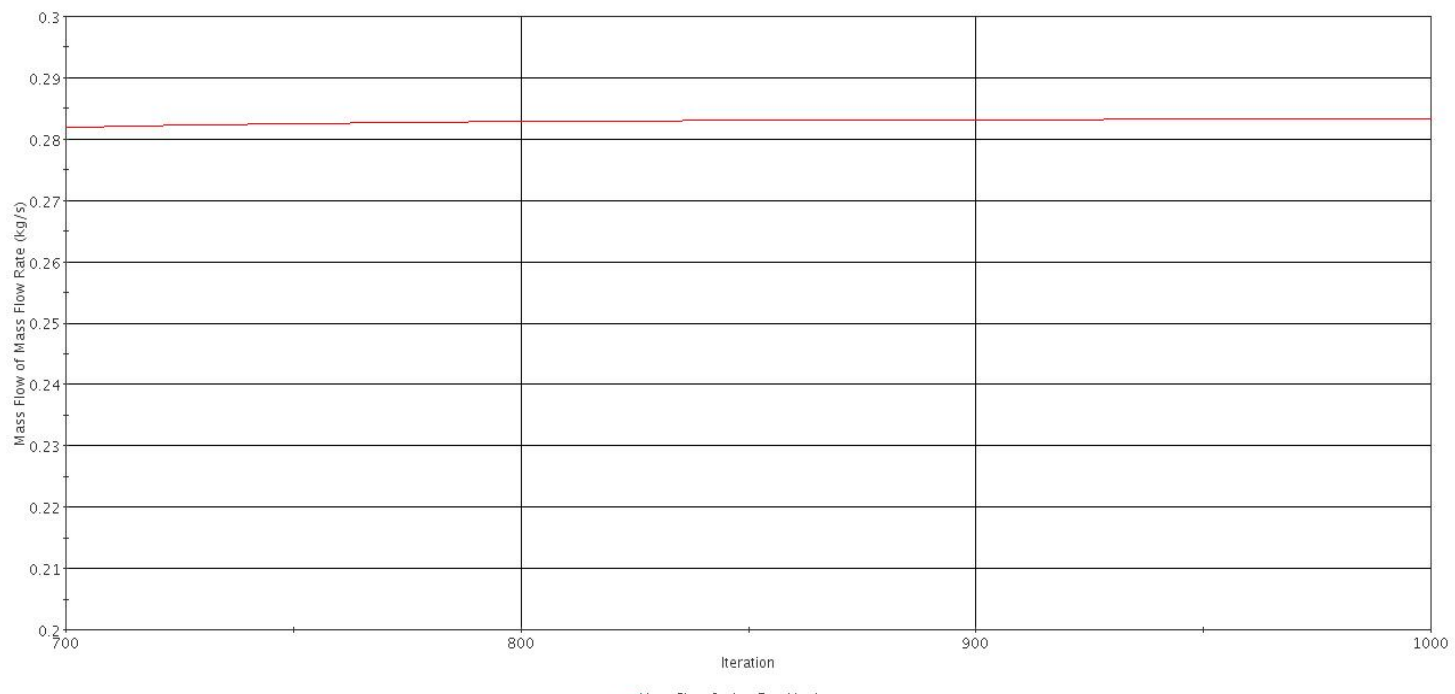

- Mass Flow Outlet Cap Monitor

Fig.39 A zoom-in view of Fig. 38

It is relatively easy for the solution to be converged for the no-crossflow-gap case but flow becomes more complicated and it is harder to meet the converging requirement once the crossflow is involved, especially when the first layer of the block is titled. Given the stopping convergence is fixed for cost-economy purpose with respect to accuracy, errors will be involved and so it is necessary to quantify them. The way to calculate the error is to run more iterations $(5,000$ for present work) until the change of the solution is negligible and compare this "accurate" solution with the one obtained after 1,000 iterations. Since this error is function of the flow pattern or differs case by case, only the cases BG6CG1_2, BG6CG1 and BG6CG0 were studied with a volumetric velocity of 180 gpm. Bypass flow fraction in percentage was chosen as the target variable to demonstrate related errors. The errors in percentage were found to be $0.64 \%$, $0.27 \%$ and $0.08 \%$ respectively. These values will be applied to all six flow rates for 
each case and obviously this is an approximation because it is not necessary or it would not be worth to run 5,000 iterations for each case with six different flow rates.

Another source of error is the discretization error which is from the truncation of higher order term in the Taylor serials when the governing equations are discretized. The discretization error can be investigated by performing the grid independence study and quantified by employing the Richardson Extrapolation Method. Fig. 40 presents the errors using error bars for the results from the grid independence study. The "High" in the legend means high mesh density. The average errors in percentage for the case BG6CG1, BG4CG1 and BG2CG1 are 3.9\%, 4.9\% and 7.0\% respectively. It is interesting to see that the case BG2CG1 has the largest error although the error bars are insignificant. This is because that the difficulties in meshing increase as the bypass flow gap becomes narrower, that is, the chance for low quality meshes existing in the model increases. Furthermore, the roundoff error cannot be neglected when the mesh size becomes small. The reason why error bars are almost invisible is simply because the solution values themselves are too small. Although the errors range from $4.9 \%$ to $7.0 \%$, the change in the bypass flow fraction is still small (around $1 \%$ to $2 \%$ ). 


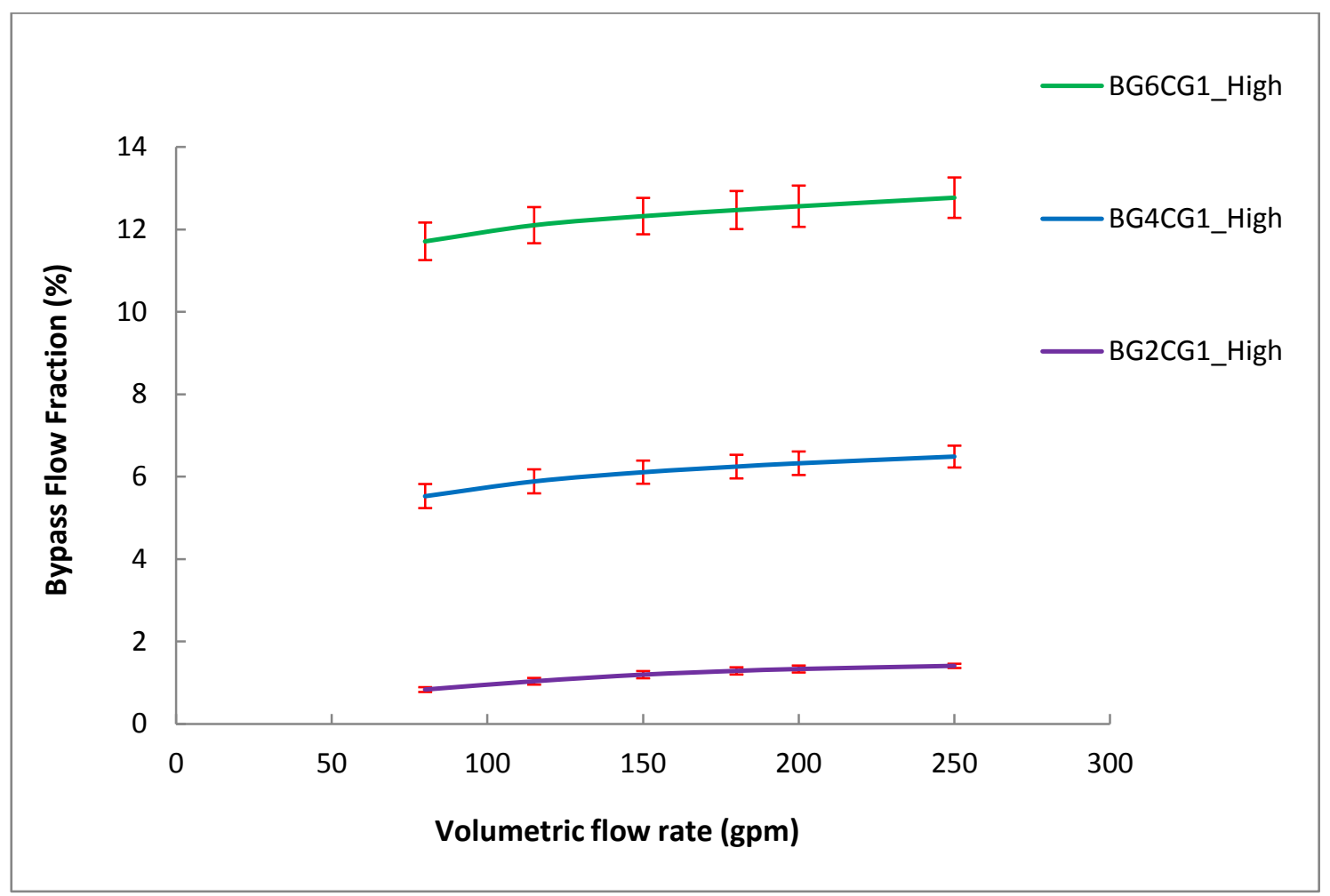

Fig.40 Grid independence study results with error bars

Thus the total errors will be the combination of the iterative convergence error and the discretization error. The general rule of calculating the error propagation or the accumulated error is described in Eq. (31).

$$
\delta w=\sqrt{\left(\frac{\partial F(\vec{u})}{\partial u_{i}} \delta u_{i}\right)^{2}+\cdots+\left(\frac{\partial F(\vec{u})}{\partial u_{j}} \delta u_{j}\right)^{2}}
$$

where $\delta w$ is the accumulated error, $\mathrm{F}(\vec{u})$ is a function of $\vec{u}, \delta u_{i} \ldots \delta u_{j}$ are independent random errors. However, this rule cannot be applied to the current problem because the discretization and the iteration are two successive events and corresponding errors are not independent random errors. The error from the discretization will be embedded into the iteration process. 
An approximation is needed before calculating the total errors. It is noticed that the iterative convergence error was evaluated by the cases which will be used to demonstrate the results including BG6CG0, BG6CG1 and BG6CG1_2. However, the discretization error was studied based on the cases BG6CG1, BG4CG1 and BG6CG0. Since the globe base size for the mesh is similar for these two sets of cases, it is reasonable to assume that the discretization errors for both sets of data are also similar. With this approximation the accumulated error can be evaluated.

Instead of using the error propagation rule the deterministic method was employed which is akin to the process of finding the probability of two successive events. As shown in Fig. 41, the value of the flow variable $\emptyset$ ranges from $\emptyset_{d}^{\max }$ and $\emptyset_{d}^{\text {min }}$ after the discretization error $\varepsilon_{\mathrm{d}}$ is introduced into the system. Then this error is brought into the iteration process and contributes to the iterative convergence error and also to the final results. By repeating the calculation with the given iterative convergence error $\varepsilon_{\mathrm{I}}$ the new upper limit value $\emptyset_{I}^{\max 1}$ and the new lower limit $\emptyset_{I}^{\min 2}$ can be obtained. These two limits would be the possible maximum and minimum values for the fluid variable $\varnothing$, that is, the simulation results could be trusted in this certain range given that the physical modeling error and other errors discussed above are neglected. 


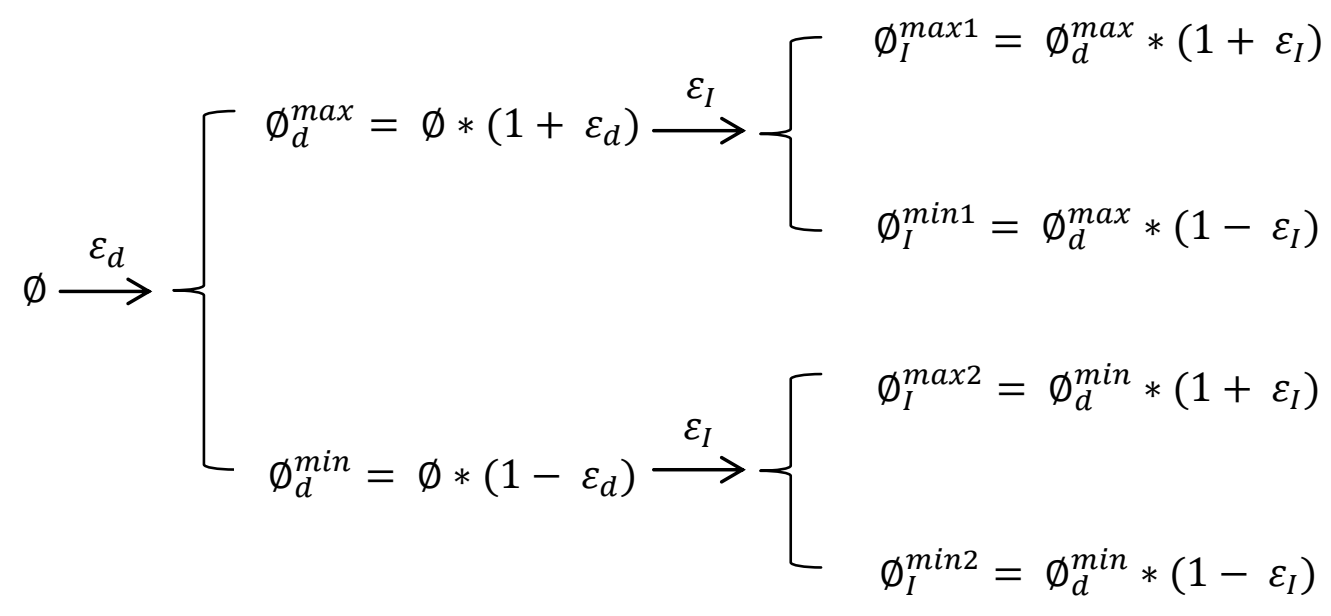

Fig.41 Flow chart of the deterministic method to calculate the accumulated errors

Fig. 42 presents same results as that in Fig. 25 but with error bars included. The average accumulated errors are about $4 \%$ relative to the results directly obtained from the simulation but the bypass flow fraction itself varies within $1 \%$. It is seen that the solutions for each case will overlap with each other if the errors are considered. This is because the crossflow gap size is only $1 \mathrm{~mm}$ and the influence to the redistribution of the coolant is still limited. That is why the differences in bypass flow fraction among these cases are small and in the same range of the errors. It is expected that differences would increase if the crossflow gap size is increased or the first layer of the fuel block is titled more. Meanwhile, the discretization error, the majority of the source of errors, would also be reduced as the meshing difficulties would be much smaller when the dimension of the gap is increased. But if the titled-crossflow-gap case and the no-crossflow-gap case are compared considering the errors, the conclusion drawn based on the results shown in Fig. 24 still holds, that is, the presence of the wedge-shape crossflow gap will 
decrease the bypass flow fraction as the coolant prefers to flow into coolant channels after passing through the crossflow gap because the flow receives less resistance in the coolant holes.

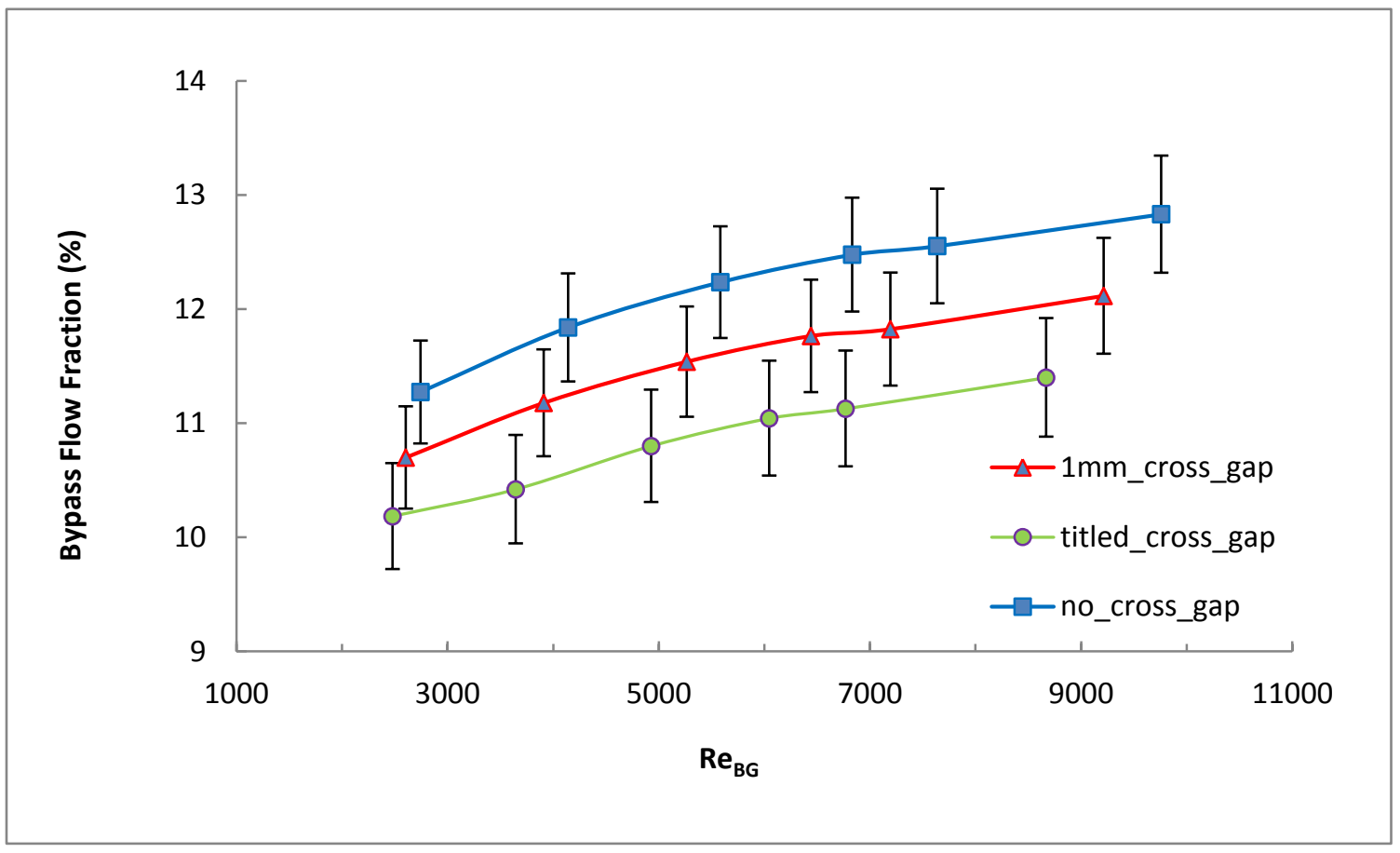

Fig.42 Bypass flow fraction as a function of $\mathrm{Re}_{\mathrm{BG}}$ with accumulated errors

However, the discretization error needs to be reduced further if simulation results are close for different cases. The possible ways to dampen the influence from the discretization include further refining of the mesh around the interested regions or very narrow regions such as crossflow gap and optimization of the prism layer thickness and the number of prism layers to make sure that the mesh is able to capture the velocity 
gradient in the boundary region. But obviously, the computational cost needs to be evaluated when the cell density is increased.

Another possible option is to change the type the mesh used in the present study or at least to try other types of meshes, such as the trimmer mesh and hexahedral mesh. But it may need the experimental data to evaluate which type of the mesh predicts the best results for the bypass flow and crossflow phenomenon in the prismatic VHTR core. 


\section{CONCLUSION}

A preliminary three-dimensional steady state simulation was performed in the commercial CFD code STARCCM+ 6.02.014 for a two-layer block of VHTR model built in the Texas A\&M University. Polyhedral mesh is applied to discretize the fluid domain and the total number of cells ranges from 10 to 50 million. Multi-region meshing technique was used to improve the meshing efficiency. The widely used k- $\varepsilon$ turbulence model was chosen for its robustness and good computational efficiency with respect to satisfying accuracy. The second-order upwind differencing scheme and two-layer all $\mathrm{y}^{+}$ wall treatment were selected to calculate the convection on the face of each cell and to blend the laws to predict the shear stress respectively. Grid independence study was performed to find the optimized the mesh size to make sure that the solution obtained is independent of mesh sizes.

The effect of bypass flow gap width and the presence of crossflow gap as well as its shape were investigated. The computational results indicated that the Reynolds number in the coolant channels could meet the design requirement of 30,000 which was close to the real condition (medium power level) in the prismatic VHTR. The bypass flow fraction was around $12 \%$ and it was found that the flow was laminar in the $2 \mathrm{~mm}$ bypass flow gap but it was turbulent for the $4 \mathrm{~mm}$ and $6 \mathrm{~mm}$ case. Also the bypass flow fraction is proportional to the Reynolds number and the bypass flow gap width. Then the influence from the crossflow gap to the distribution of the coolant in the system was studied. It was observed that the presence of the crossflow gap and its shape had a 
significant effect on the redistribution of the flow when the coolant passes through the crossflow gap. A significant secondary flow moving from the bypass flow gap towards coolant holes was found, which resulted in a up to $28 \%$ reduction of the coolant mass flow rate in the bypass flow gap while the value for flow in the coolant channels increased by around 5\%. The pressure distributions in the crossflow gap region and in the coolant channel were evaluated in a non-dimensional way and results demonstrated that pressure in the bypass flow gap was much higher than that in the coolant channels. The pressure drop at the inlet due to the sudden contraction in area could be around 1kpa while the value was about $180 \mathrm{~Pa}$ around the crossflow gap region.

Two major errors, discretization error and iterative convergence error, were quantified based on the grid independence study and a deterministic method. The iterative convergence errors in percentage range from $0.08 \%$ and $0.64 \%$ which were evaluated based on the variation of the crossflow gap shape. The discretization error was calculated using Richardson Extrapolation Method for the uniform crossflow gap but with varied bypass flow gap sizes. The errors for the case BG6CG1, BG4CG1 and BG2CG1 were $3.9 \%, 4.9 \%$ and $7.0 \%$ respectively. Assumption was made to treat the discretization error obtained from the uniform crossflow gap case as the same as that for the no-crossflow-gap case and the wedge-shape case so that these two errors could be combined. However, the rule of error propagation cannot be applied in calculating the accumulated errors because those two errors are not independent random errors. Instead, the deterministic method was used to determine the upper and lower limit of the solution when errors were introduced. The average total errors were around $4 \%$. For future work, 
the discretization error needs to be reduced so that the bypass flow fraction for varied cases could be distinguished. In addition, other turbulence models should be studied to find which model predicts the best results after comparing the data with experimental results. 


\section{REFERENCES}

AIAA, 1998. "Guide for the Verification and Validation of Computational Fluid Dynamics Simulations.” AIAA Guide G-077.

Bird, R. B, Stewart, W.E., Lightfoot, E. N., 2006. "Transport Phenomena.” New York, John Wiley \& Sons, Inc.

Celik, I.B., Ghia, U., et al., 2008. "Procedure for Estimation and Reporting of Uncertainty Due to Discretization in CFD Applications." ASME Journal of Fluids Engineering, Vol. 130, 078001-4.

ERCOFTAC, 2000. "Best Practice Guidelines, Version 1.0", ERCOFTAC Special Interest Group on Quality and Trust Industrial CFD, http://www.ercoftac.org/publications/ercoftac_best_practice_guidelines/

FLUENT 12.0, 2009. ANSYS, Inc., 275 Technology Drive Canonsburg, PA 15317. http://www1.ansys.com/customer/content/documentation/121/fluent/flth.pdf

Fox, R. W. 2006. "Introduction to Fluid Mechanics.” 6th edition, Wiley, John \& Sons. Inc., Hoboken, NJ.

Groehn, H. G. 1980. "Disturbance of the Cooling Gas Distribution in HTGR Fuel Blocks due to Bypass Flow." Proceedings of ANS/ASME/NRC International Topical Meeting on Nuclear Reactor Thermal-Hydraulics, NY, USA, October 5-8.

Johnson, R.W., Sato, H. 2012. "Bypass Flow Computations Using a One-twelfth Symmetric Sector for Normal Operation in a 350 MWth Prismatic VHTR." Nucl. Eng. Des. http://dx.doi.org/10.1016/j.nucengdes.2011.10.070 .

Kaburaki, H. and Takizuka, T. 1985. "Leakage Flows in High-Temperature Gas-Cooled Reactor Graphite Fuel Element." Journal of Nuclear Science and Technology, vol.22, pp.387-397. 
Kaburaki, H. and Takizuka, T. 1987. "Effect of Crossflow on Flow Distribution in HTGR Core Column." Journal of Nuclear Science and Technology, vol.24, pp.516-525.

Kaburaki, H. and Takizuka, T. 1990. "Crossflow Characteristics of HTGR Fuel Blocks." Nucl. Eng. Des., vol.120, pp.425-434.

Kim, M. H., Lim, H. S. 2011. "Evaluation of the Influence of Bypass Flow Gap Distribution on the Core Hot Spot in a Prismatic VHTR Core." Nucl. Eng. Des. 241, 3076-3085.

MacDonald, P.E., 2003. "NGNP Point Design—Results of the Initial Neutronics and Thermal-Hydraulic Assessments." INEEL/EXT-03-00870, Rev. 1, Idaho Falls, Idaho, September, 2003.

NASA, 2012. "NPARC Alliance CFD Verification and Validation", http://www.grc.nasa.gov/WWW/wind/valid/homepage.html.

Ortensi, J., Cogliati, J.J., et al. 2010. "Deterministic Modeling of the High Temperature Test Reactor." Proceedings of HTR 2010, Prague, Czech Republic, October 1820, 2010, Paper 178.

Olson, H. G., Brey, H. L., and Warembough, D. W. 1982. "The Fort St. Vrain High Temperature Gas-Cooled Reactor: X. Core Temperature Fluctuations.” Nucl. Eng. Des., vol.72, pp.125-137.

Patankar, S. V. 1980. "Numerical Heat Transfer and Fluid Flow." Hemisphere Publishing Corporation, Washington, D.C.

Sato, H., Johnson, R., and Schultz, R. 2010. "Computational Fluid Dynamic Analysis of Core Bypass Flow Phenomena in a Prismatic VHTR." Annals of Nuclear Energy, vol.37, pp.1172-1185. 
Schultz, R. R. 2011. "Bypass Flow in Prismatic VHTR: Summary of I-NERI R\&D.” VHTR R\&D FY11 Technical Review Meeting, April 26-28, Albuquerque, New Mexico.

Shih, T. H., Liou, W. W., Shabbir, A., Zhu, J. 1995. “A New k-E Eddy-Viscosity Model for High Reynolds Number Turbulent Flows - Model Development and Validation." Computers Fluids, 24 (3), 227-238.

CD-Adapco Group, 2008. Star-CCM+ User Guide. Version 6.04.014, Melville, NY

Tak, N.I., Kim, M.-H., and Lee, W. J. 2008. "Numerical Investigation of a Heat Transfer within the Prismatic Fuel Assembly of a Very High Temperature Reactor." Annals of Nuclear Energy, vol.35, pp.1892-1899, 2008.

Tung, Y. H., Johnson, R. W., Sato, H. 2011. "Effects of Graphite Surface Roughness on Bypass Flow Computations for an HTGR." ASME 2011 Pressure Vessels and Piping Division Conference, Baltimore, Maryland, July 17-21, 2011

Versteeg, H. K., Malalasekera, W. 2007. "An Introduction to Computational Fluid Dynamics: The Finite Volume Method", $2^{\text {nd }}$ Edition, Prentice Hall Limited, Upper Saddle River, NJ, 2007

Yoon, S. J. Jin, C.Y, et al. 2011. "Study on the Flow Distribution in Prismatic VHTR Core with a Multi-block Experiment and CFD Analysis." Nucl. Eng. Des. 241, 5174-5182.

Yoon, S.J., Lee, J.H., et al. 2012. "The Effects of Crossflow Gap and Axial Bypass Gap Distribution on the Flow Characteristics in Prismatic VHTR Core." Nucl. Eng. Des. http://dx.doi.org/10.1016/j.nucengdes.2012.04.025. 


\section{PART II: EXPERIMENTAL STUDIES}

\section{INTRODUCTION}

The bypass flow in a prismatic VHTR is an important safety concern. This flow refers to the coolant that bypasses the coolant channels where the cooling is more efficient. Although higher bypass flow fraction is able to cool down the peripheral areas, it implies that the hot spots may appear in the normal operation or accident scenarios as less coolant pass the coolant holes. To better understand the bypass flow phenomenon and validate the numerical results obtained previously, an experimental investigation was performed in which the air was pumped through a two-block VHTR model. Furthermore, the non-intrusive technique Laser Doppler Velocimetry (LDV) was used to capture the velocity information inside the bypass gaps.

\section{EXPERIMENTAL SETUP}

As shown in Fig.43, the experimental loop mainly consists of a $7.5 \mathrm{hp}$ blower, four velocity meters (Model 8360, TSI), a 24-jet collision nebulizer or atomizer (BGI), two-block VHTR model, 3D traverse system and the LDV system (Dantec). The detailed information of the VHTR model can be found in the reference (H. Wang, 2012). Velocity Meter \#1 measures the velocity of the inlet flow from which the total flow rate can be calculated. Other three ones are used to obtain the flow rate of each single collector. The bypass gap width is $6 \mathrm{~mm}$ for this experiment. 


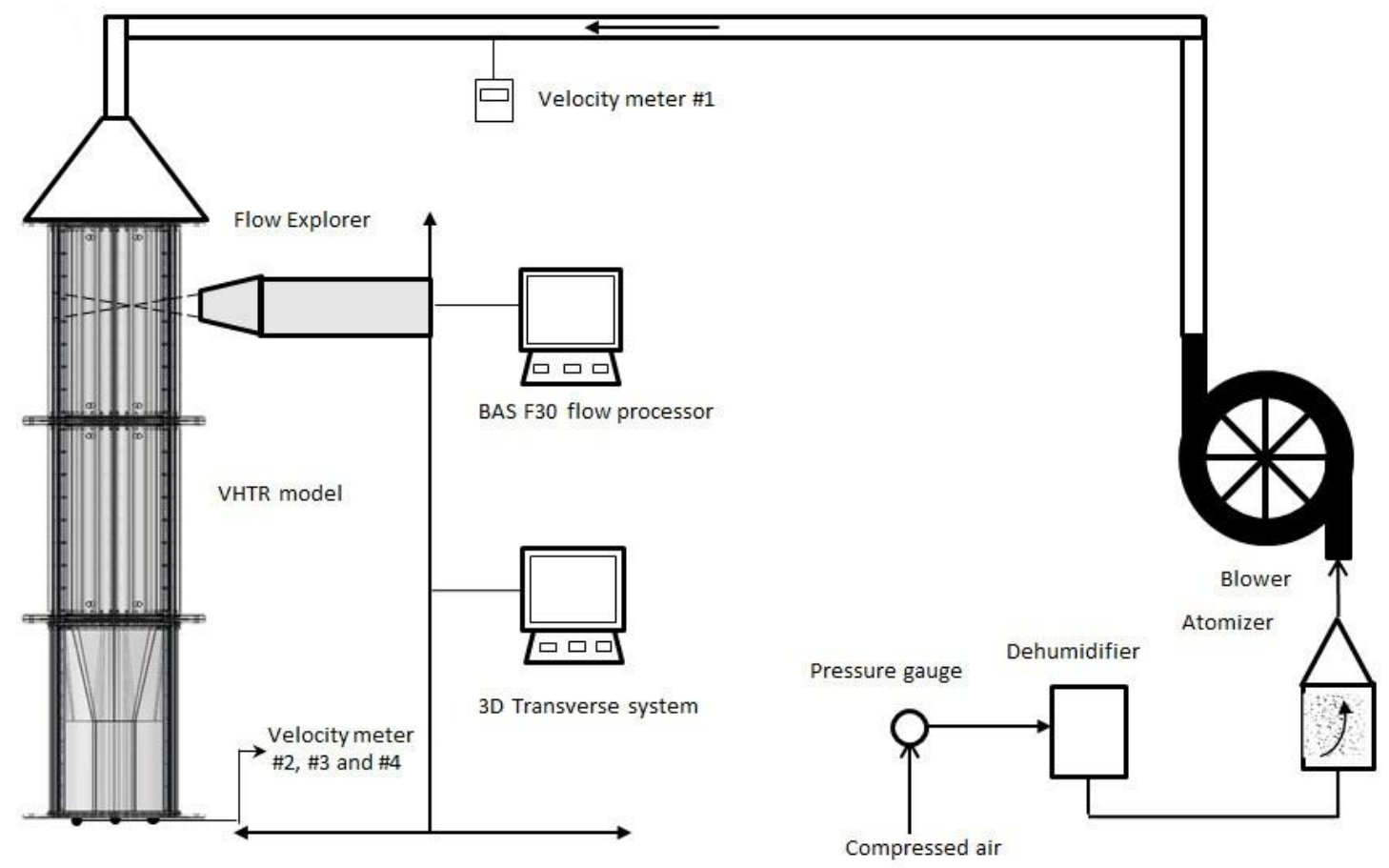

Fig.43 Experimental loop

As shown in Fig. 44, improvements of the facility have been made by replacing the upper plenum and the supporting structure with newly designed structures. The problems of the previous design include but not limited to leakages in the junction area between the upper plenum and the VHTR model, uneven distribution of the flow due to the design defect and the vibration of the whole facility because of the instability of the previous supporting structure. This small vibration increases the uncertainty of the precise LDV measurements. 


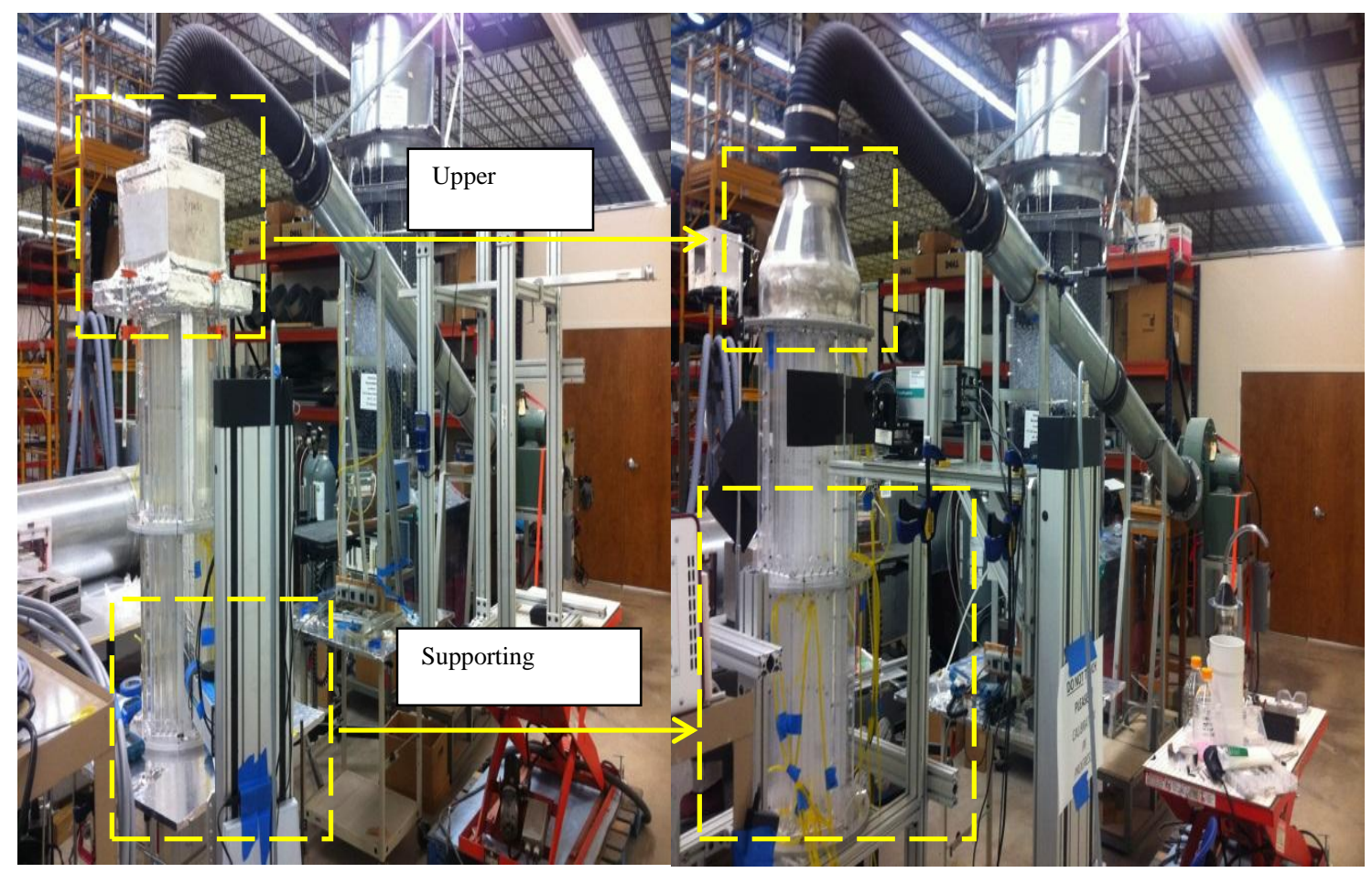

Fig.44 Improvements of the upper plenum and the supporting frame

Another two major modifications to the previous design are on the observing window and the flow conditioner. At the first beginning, no signals could be obtained within the gap region because of some unknown reasons. Different LDV systems including one from TSI and different atomizers (Both of them can be seen in Fig.45) were tried but have had no success. Finally it was found that the gap divider which also serves as the observing window was polished leaving an unsmooth surface. It was still very transparent to human eyes but not to the laser. Signals could be observed after the unpolished ones with smooth surface were installed. A flow conditioner or straightener is important in such an experiment in which the flow distribution has a significant 
influence on the results. Previously, the conditioner was located at the bottom of the upper plenum as indicated in Fig. 46 (left). It was contacting the inlet of the VHTR model closely. The amount of the flow received by the coolant channels and bypass gaps strongly depended on the grid density of the flow conditioner right on the top of the flow channels. To eliminate this external influence to the coolant distribution, a new flow conditioner shown in Fig. 46 (right) was installed at the top or the inlet of the upper plenum so that the flow was able to mix within the chamber before entering the facility.

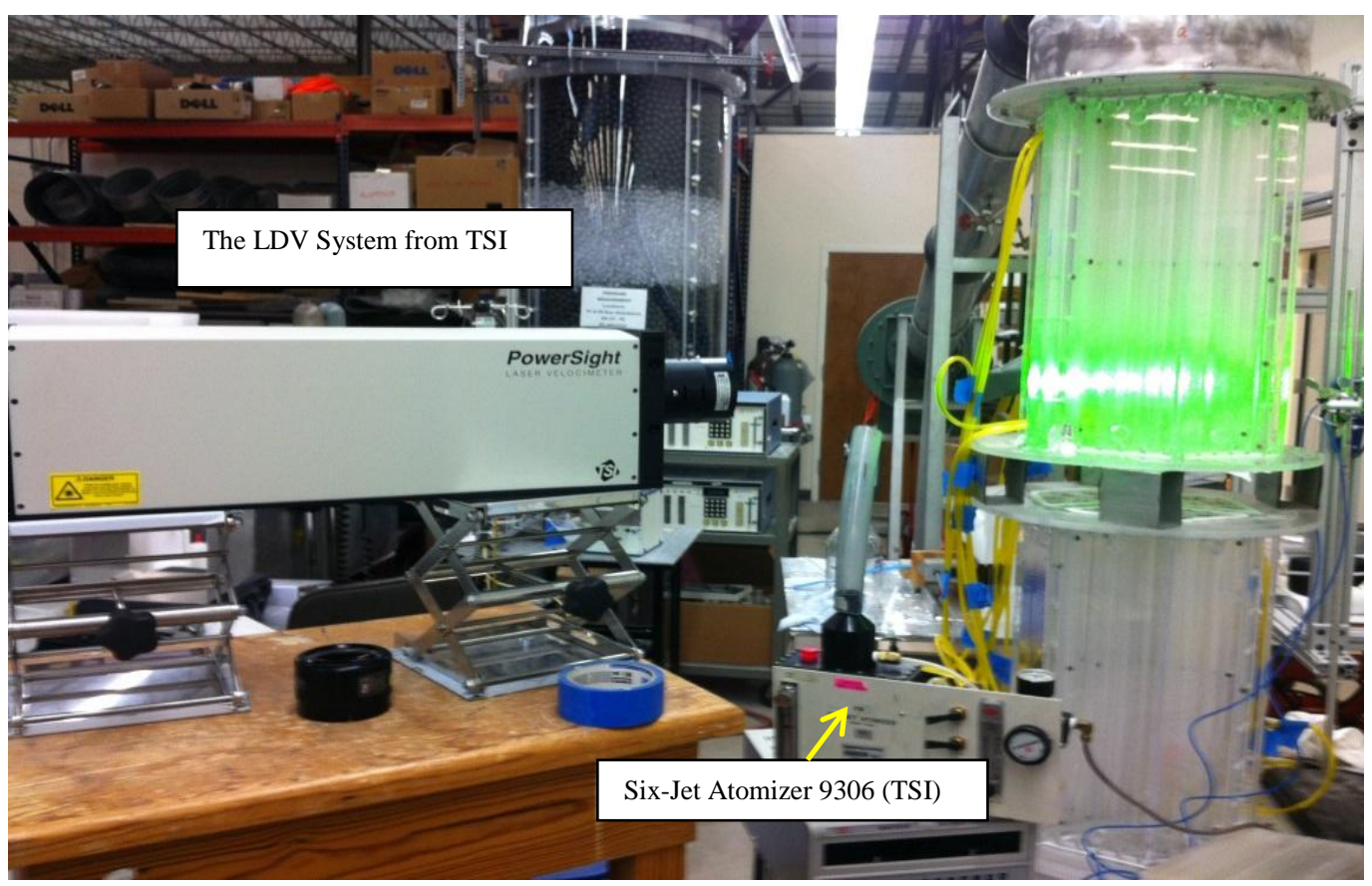

Fig.45 TSI LDV system borrowed for test purpose 

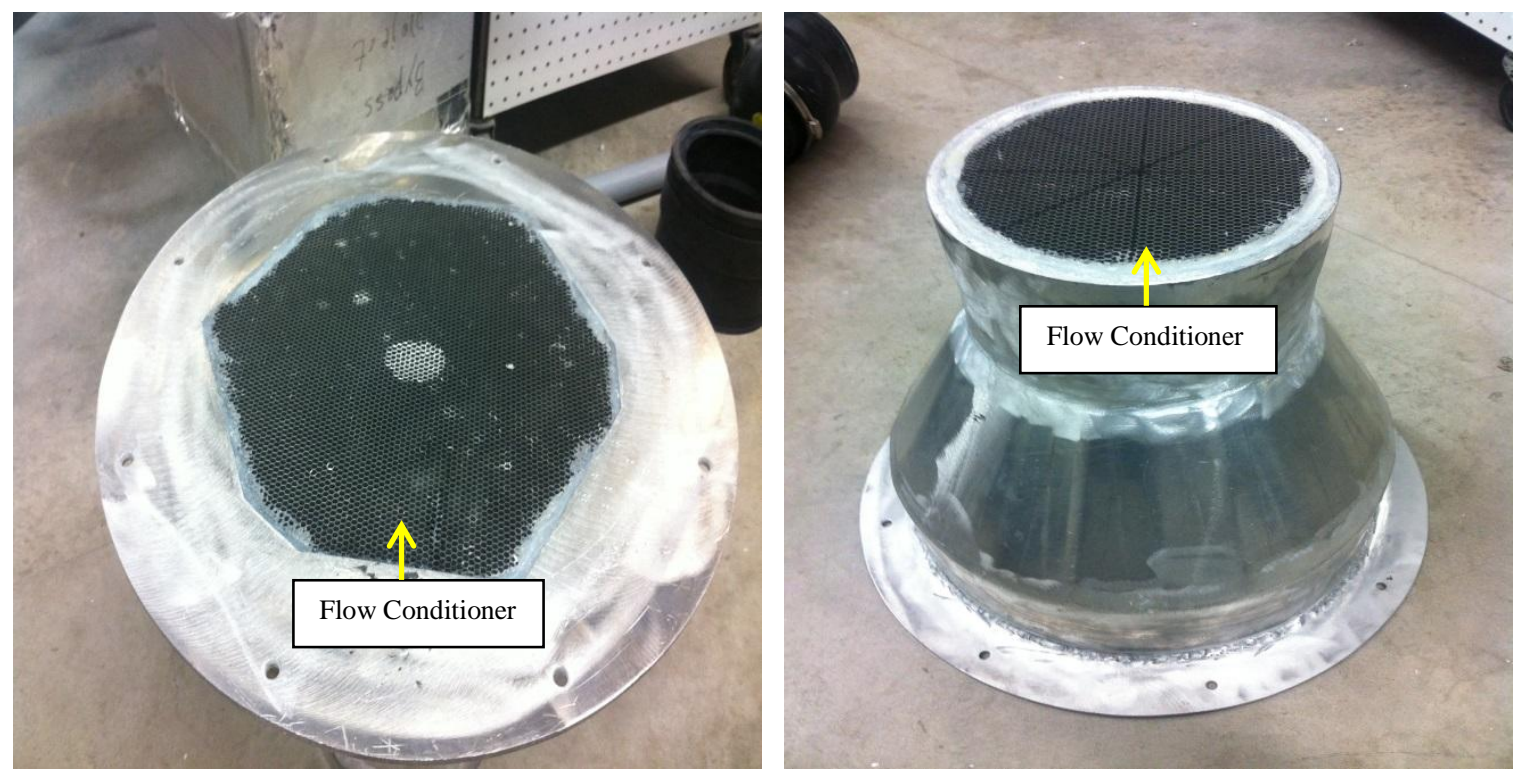

Fig.46 Flow conditioner in previous design (left) and new design

\section{MEASUREMENT TECHNIQUE}

\subsection{LDV measurement}

The principle of LDV (Fringe model) is based on the Doppler frequency shift caused by the particles passing through the intersection (measuring volume) of two coherent laser beams as shown in Fig. 47. The Dantec LDV optics used in this work has a back-scattering arrangement. The focal length of the two laser beams with frequencies of $660 \mathrm{~nm}$ and $785 \mathrm{~nm}$ is $300 \mathrm{~mm}$. The laser beams has a diameter of $2.5 \mathrm{~mm}$ and $35 \mathrm{~mW}$ power. For each laser beam, it is split into two beams after passing an $80 \mathrm{MHz}$ Bragg cell and intersects with each other at a half-crossing angle of $0.511^{\circ}$. Only 1-D measurements were performed in this work considering the small size of the gap. 


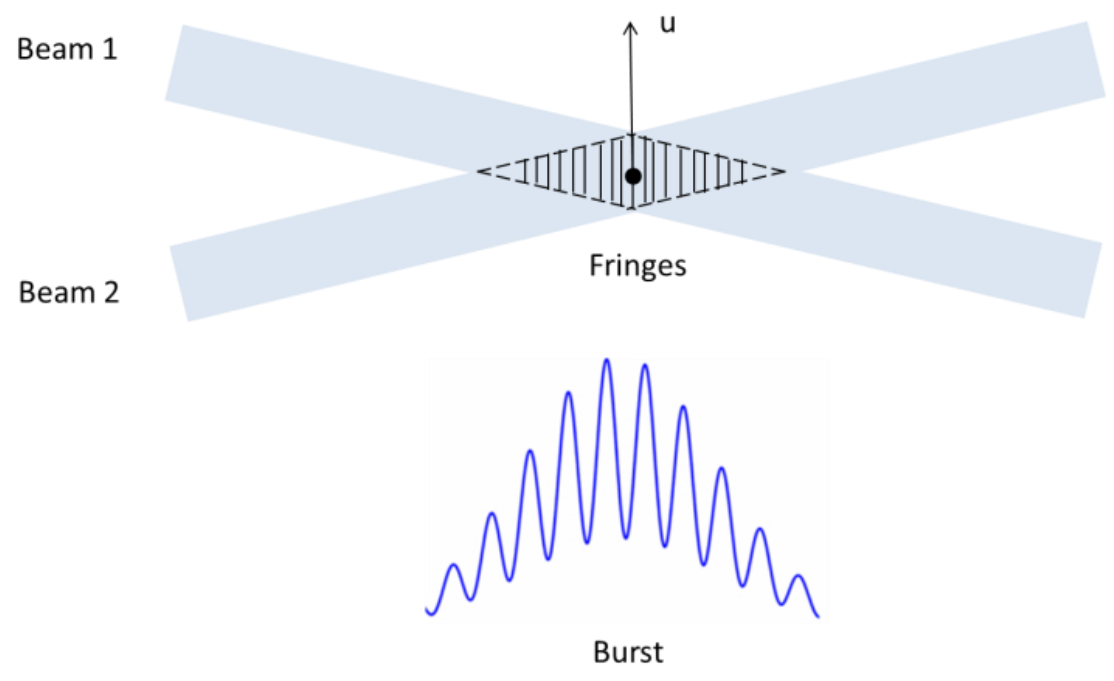

Fig.47 Principle of LDV (Fringe Model)

\subsection{Particle seeding}

The most challenging part of applying LDV in this experiment facility is the seeding. The difficulties lie in the limited portion of flow in the bypass gaps and the optical interference from the scattered lights within the setup. The seeding particle used is polystyrene latex (PSL) with an average diameter of 0.5 microns. Although these perfect spheres are almost ideal for the LDV measurements considering the size, density and the refractive index, some extra works need to be done to get good and valid signals such as the injection of the particles. The best position is the intake of the blower because for other possible places, the back pressure created by the main flow will stop the seeds from entering the loop. For example, no signals could be obtained if the 
particles were injected from the middle of the inlet pipe using a fluidized bed or from the top of the upper plenum as shown in Fig. 48.
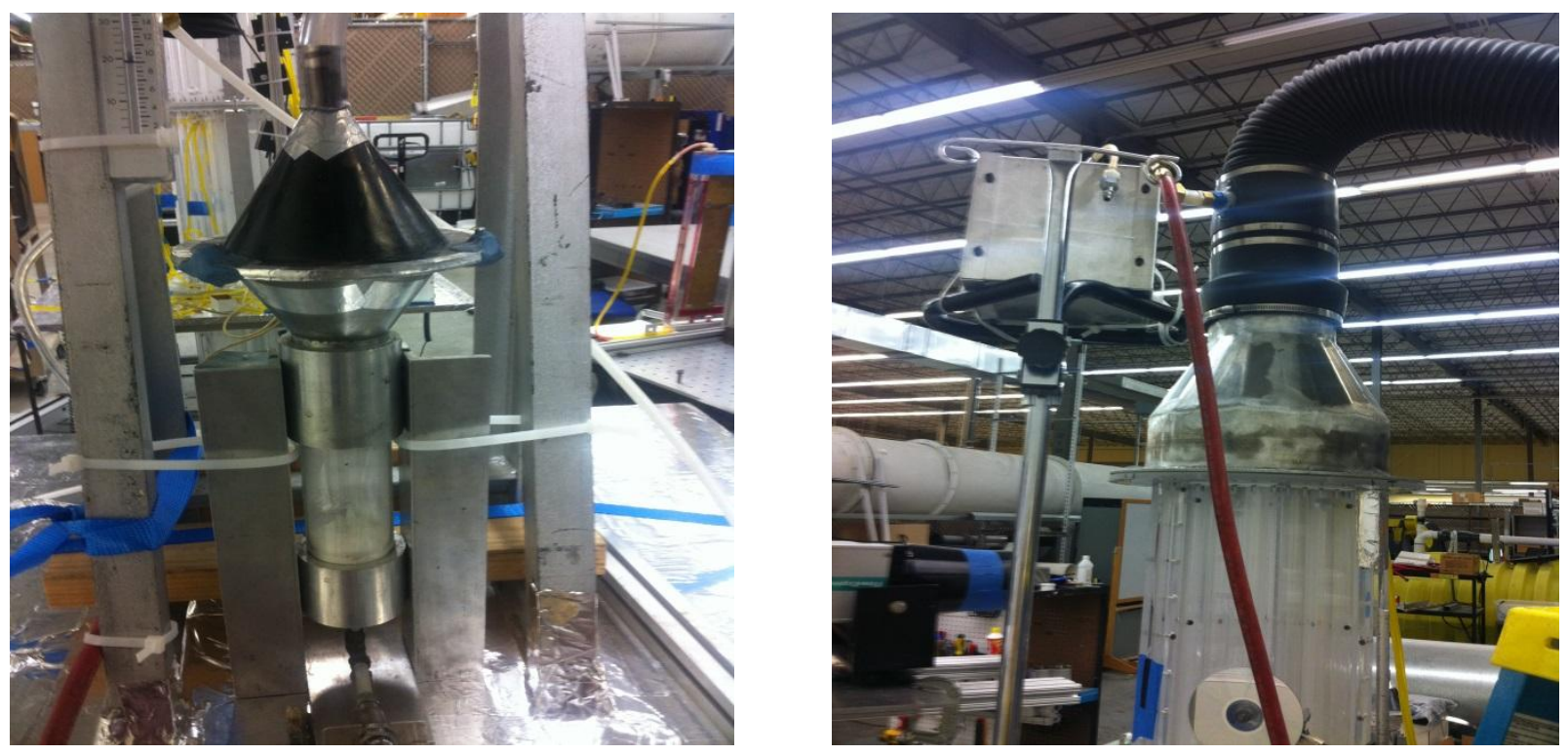

Fig.48 Particle injecting methods which did not work due to the backpressure: Fluidized bed (left) and Top injection (right)

Before using PSL, other particles such as baby powder, starch powder, chalk powder and glycol fog were tried but none of them generated good signals. The starch power is flammable so it is not as good option when injecting from the blower intake because any sparks inside the blower may cause explosion. The baby power and glycol fog generated from a fog machine shown in Fig. 49 (right) are mixtures of some known and unknown chemicals, which are not ideal for the LDV measurement. Furthermore, the contamination to the observing window is another issue that these powders suffer from. As for the chalk power used before in this experiment, the main issue was that its sizes varied too much because these powers were self-made using a grinder. Besides the 
size, their high density is another concern. The size distribution of the chalk power measured by aerodynamic particle sizer (APS) spectrometer (TSI) is shown in Fig. 49 (left).
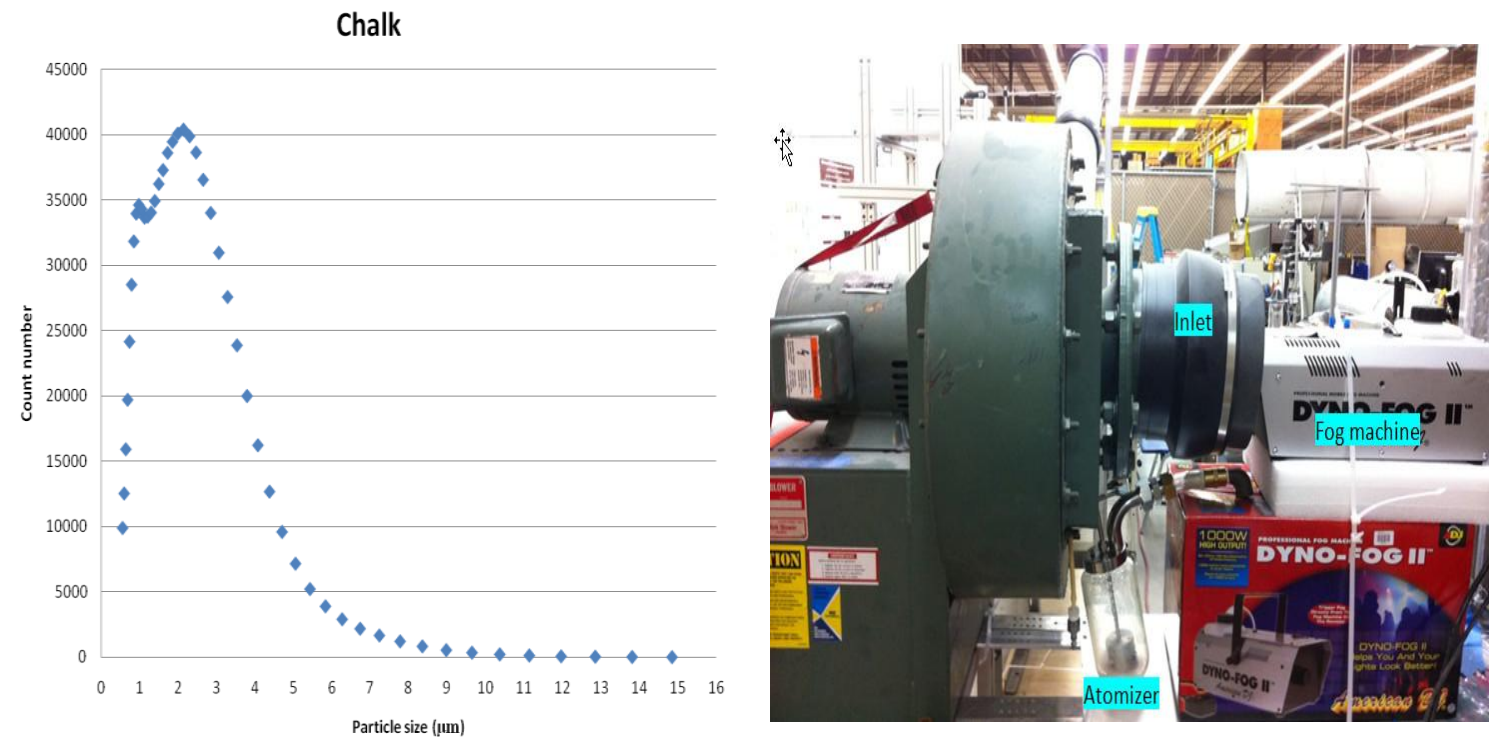

Fig.49 Size distribution of the chalk power (left) and the Fog machine (right) None of these two is good for LDV measurements

\section{RESULTS}

\subsection{Pressure drop}

Static pressures were measured in the instrumental rhomboid using Manometer.

The pressure drops along the measured coolant channel (abbreviated as $\mathrm{CH}$ ) and the bypass gap $(\mathrm{BG})$ in Block $1\left(\Delta \mathrm{P} \_\mathrm{B} 1 \_\mathrm{CH}, \Delta \mathrm{P} \_\mathrm{B} 1 \_\mathrm{BG}\right)$ and in Block $2\left(\left(\Delta \mathrm{P} \_\mathrm{B} 2 \_\mathrm{CH}\right.\right.$, $\left.\Delta \mathrm{P} \_\mathrm{B} 2 \_\mathrm{BG}\right)$ as a function of Reynolds number in a random collector $\mathrm{A}\left(\mathrm{Re}_{\mathrm{CA}}\right)$ are 
shown in Fig. 50. Since the average velocities in the coolant channels and the bypass gaps are not known, the Reynolds number of collector A is chosen here as a reference. Generally the pressure drops are small which is similar as what was predicted from the CFD analysis. The discrepancies of pressure drops between the coolant channel and the bypass gap, which needs to be further investigated, may be resulted from the geometry arrangement and the measuring points.

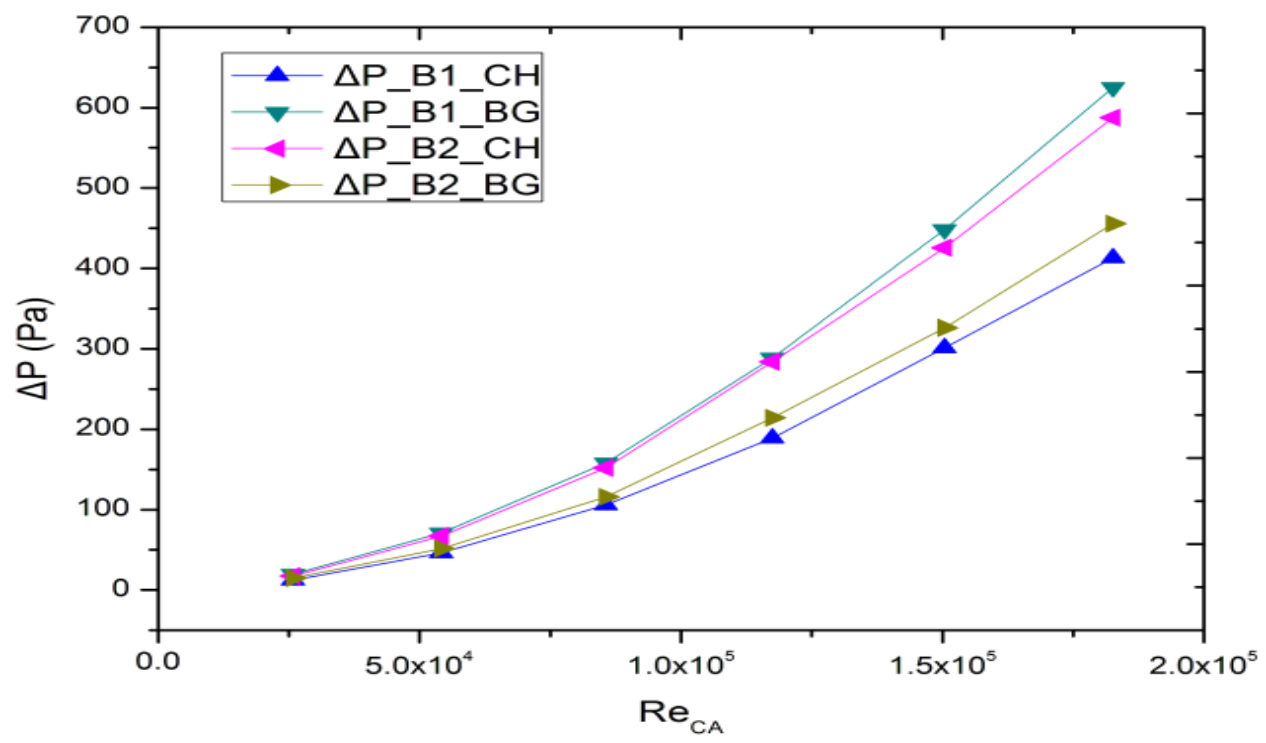

Fig.50 Pressure drop vs. $\operatorname{Re}_{\mathrm{CA}}$

\subsection{Bypass flow fraction}

The calculation of the bypass flow fraction is based on the readings of those four velocity meters. As seen in Fig. 51, the results are difference as predicted by the simulation from which the bypass flow fraction is proportional to the total flow rate or 
Reynolds number. However, the bypass flow fraction is not a strong function of the Reynolds number in the experiment. The errors from the readings of the velocity meters

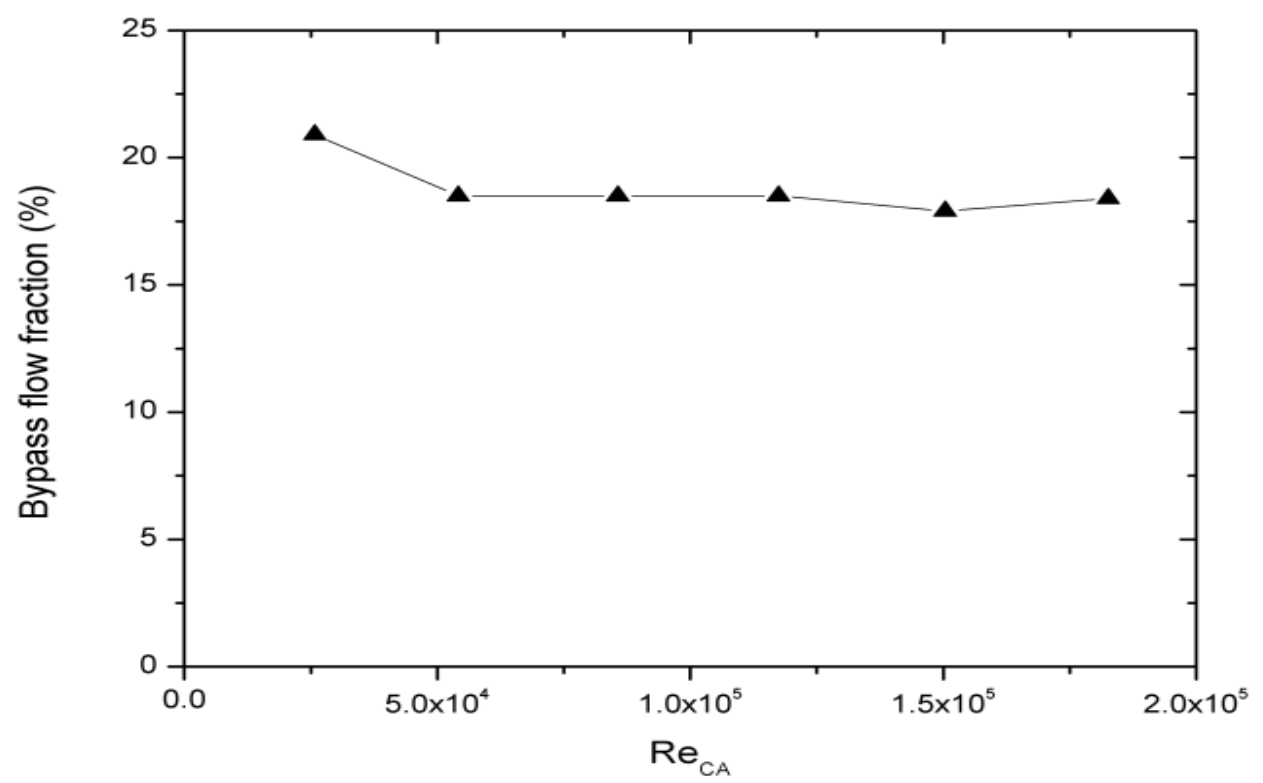

Fig.51 Bypass flow fraction vs. $\operatorname{Re}_{\mathrm{CA}}$

are discussed in the next session.

\subsection{LDV measurement results}

\subsubsection{Proof-of -concept study}

The initial failure of acquiring signals in the bypass gap necessitated the proofof-concept study of the LDV system. A DVD disc was mounted on a DC motor whose rotating speed was controlled by a power supply. A human hair, serving as a particle, was glued at the edge of the disc. By locating the measuring volume on the hair and counting the disc rotating speed (RPM (RPM), the theoretical velocity of the hair could 
be obtained which was compared with the results from LDV. The results show a good agreement as shown in Fig. 52.

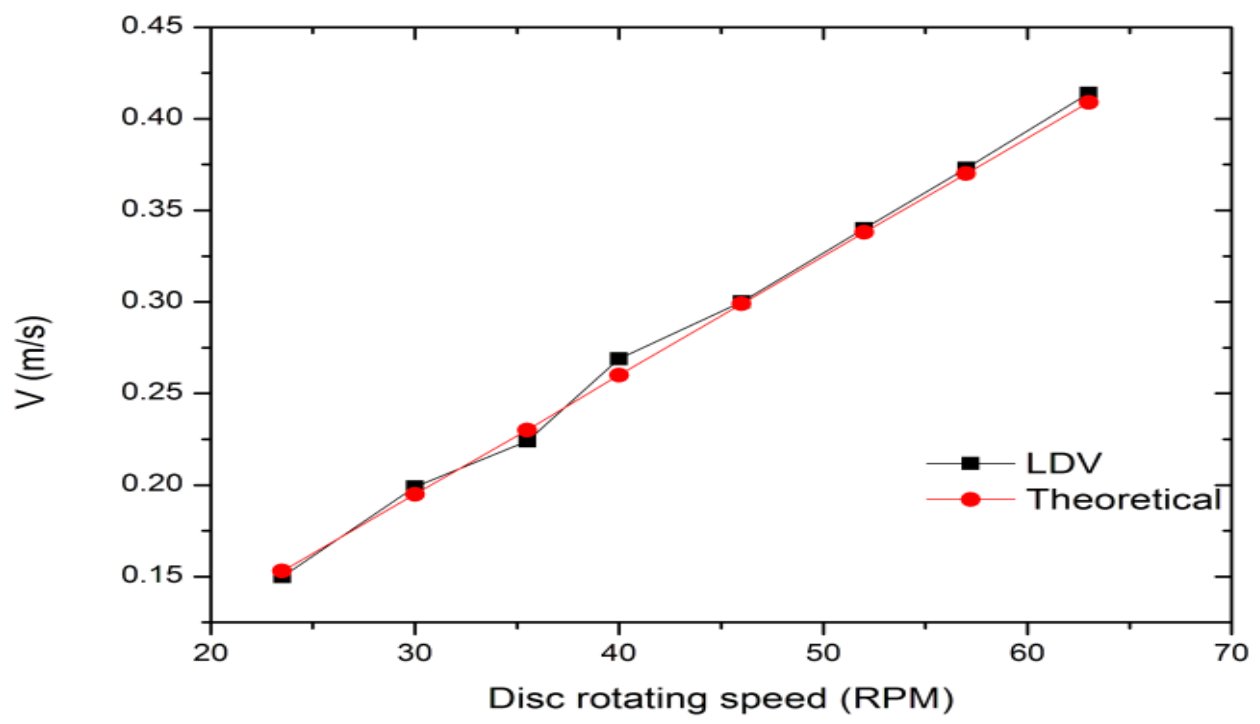

Fig.52 Proof-of-concept study results

\subsubsection{Velocities in the bypass gap}

Velocity distribution in the bypass gap was measured at $136 \mathrm{~mm}(\mathrm{P} 1)$ and 486mm (P2) away from the entrance of the bypass gap or coolant channels in the vertical or $\mathrm{z}$ direction. Both P1 and P2 are located in the first block shown in Fig. 11(left). At each position, thirteen points along one bypass gap indicated in the Fig. 53 (right) were measured. The first point is $20 \mathrm{~mm}$ from the outer edge or the gap divider. No closer than $20 \mathrm{~mm}$ from the wall or the gap divider were measured because of the poor signal to noise ratio (SNR) resulted from the wall effect. The distance between each remaining point is $10 \mathrm{~mm}$ except the last two have a distance of $8 \mathrm{~mm}$. The last point is right at the 
center of the three bypass gaps. Three Reynolds numbers $\mathrm{Re}_{\mathrm{BG}}$ calculated from the measured average velocity in the bypass gap (at P2 position) 4,820, 9,990 and 15,633 were tested with respect to the maximum velocity the LDV system could measure. The results are shown in Fig. 54.

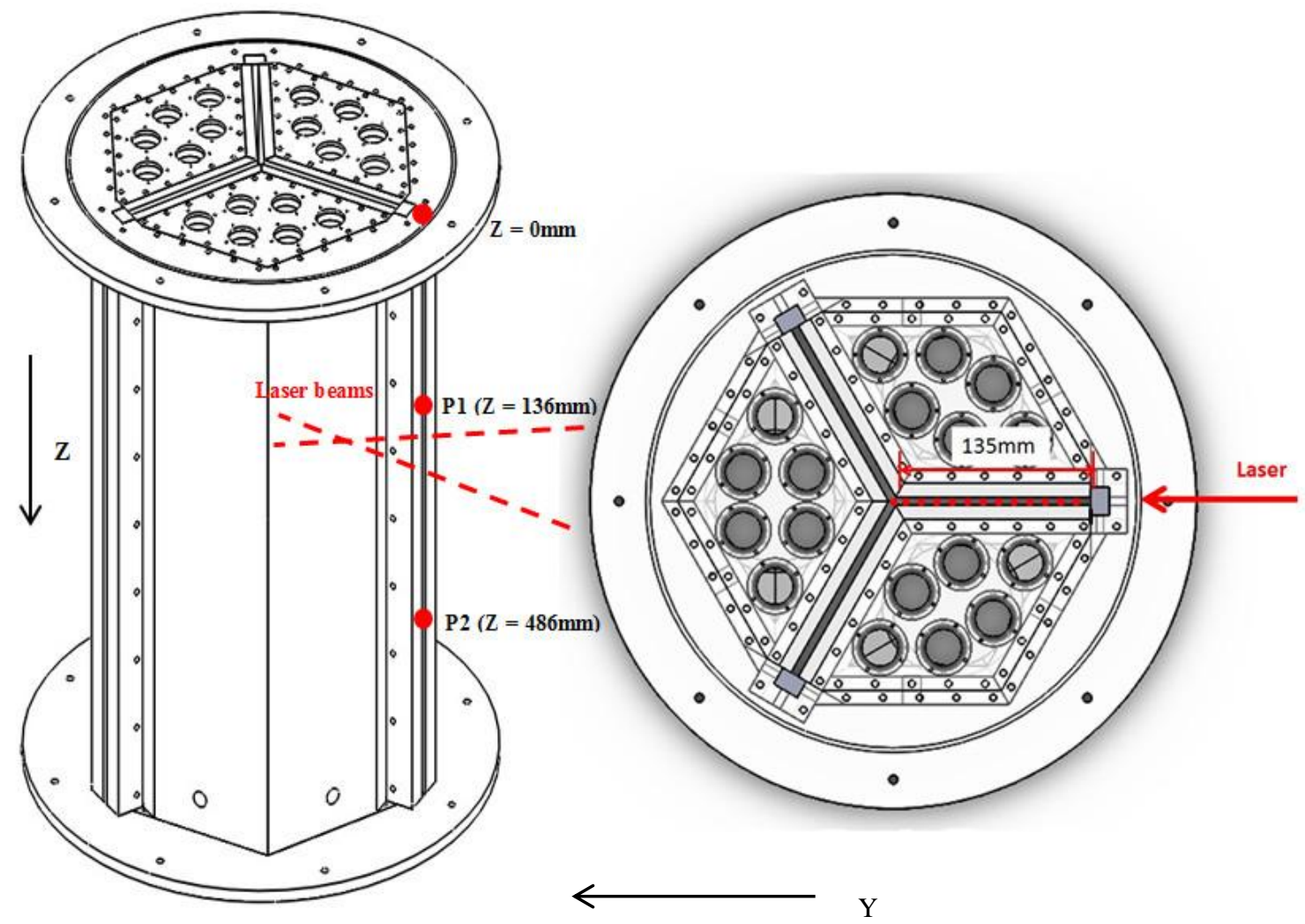

Fig.53 Illustration of the measuring positions 


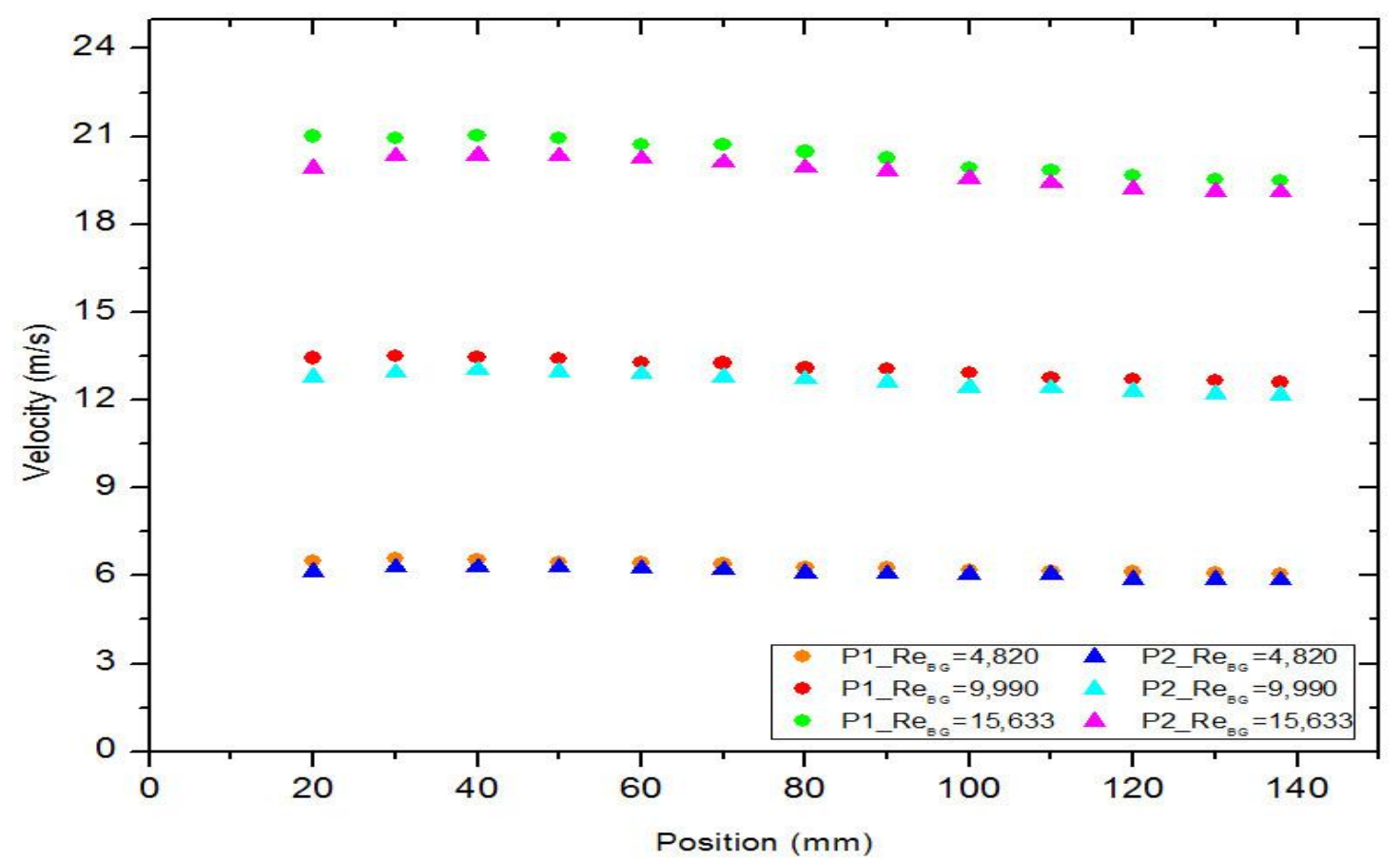

Fig.54 Velocities in the bypass gaps

Based on the Reynolds number in the bypass gaps, it can be concluded that the flows in the gaps are turbulent. This also can be verified from the velocity profiles which are almost flat. The velocities at P1 and P2 for the same $\mathrm{Y}$ are close although they are supposed to be the same in theory. This discrepancy may come from any small leakages along P1 and P2 and the cross sectional area differences. The later reason is more possible because the gap size cannot be maintained constant due to some unavoidable error from the fabrication and installation. Actually, this uneven gap sizes do not only exist in the vertical direction $(\mathrm{Z})$ but also in the horizontal direction $(\mathrm{Y})$. And that is why 
the velocities along the $\mathrm{Y}$ direction continue to decrease with the increase of $\mathrm{Y}$. The measured gap sizes at the measuring points are summarized in Table 6.

Table. 6 Variations of the target bypass gap along the Y direction

\begin{tabular}{|c|c|c|c|c|c|c|c|c|c|c|c|c|}
\hline $\mathrm{Y}(\mathrm{mm})$ & 20 & 30 & 40 & 50 & 60 & 70 & 80 & 90 & 100 & 110 & 120 & 130 \\
\hline Gap width $(\mathrm{mm})$ & 5.90 & 5.79 & 5.89 & 5.89 & 5.98 & 5.99 & 6.01 & 6.04 & 6.07 & 6.15 & 6.31 & 6.37 \\
\hline Average Gap width(mm) & \multicolumn{10}{|c|}{6.03} \\
\hline
\end{tabular}

\subsubsection{Hand-on skills to reduce the errors}

Although the LDV measurement is one of most accurate method to obtain the velocity data, it is true only when this technique is applied correctly and properly. Possible errors involved in this technique come from the seeding particles selected, system alignment, scattered light interference, wall effect and system parameter selections such as sensitivity and total number of samples. The seeding issue has been discussed previously. 500 samples were collected for each case. Larger number of samples should be chosen but the PSL is extremely expensive. So these two conflicts should be compromised.

\subsubsection{Optics alignment}

In this experiment, optical alignment was taken very carefully and precisely. Firstly, the crossing condition of the two beams (1D) or four beams (2D) was exanimated by placing a 10X objective around the measuring volume as shown in Fig. 
55. By moving the objective and observing the change of the beam shape on the wall, one can determine if the beam crossed perfectly. If a single and small dot could be observed on the wall during the moving the objective, then the beams cross well. The following work is to make sure the laser is shooting against the wall perpendicularly. The simplest way to check this is to cut a small hole in a paper or card so that one of the beams can exit this hole as shown in Fig. 56 and observe the position of the reflected beam on the card. This reflection should be in line vertically and horizontally with the transmitted beam.

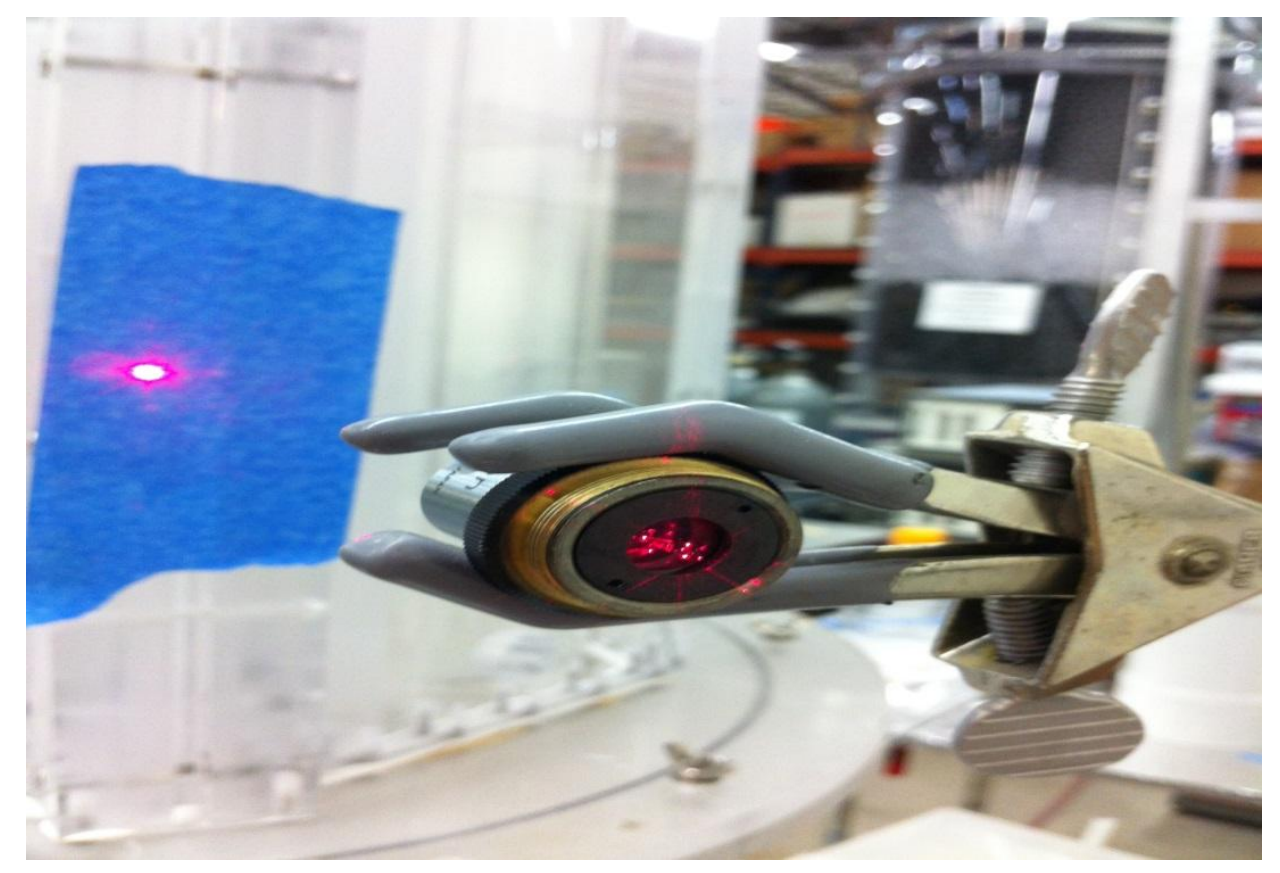

Fig.55 Beam crossing condition test 


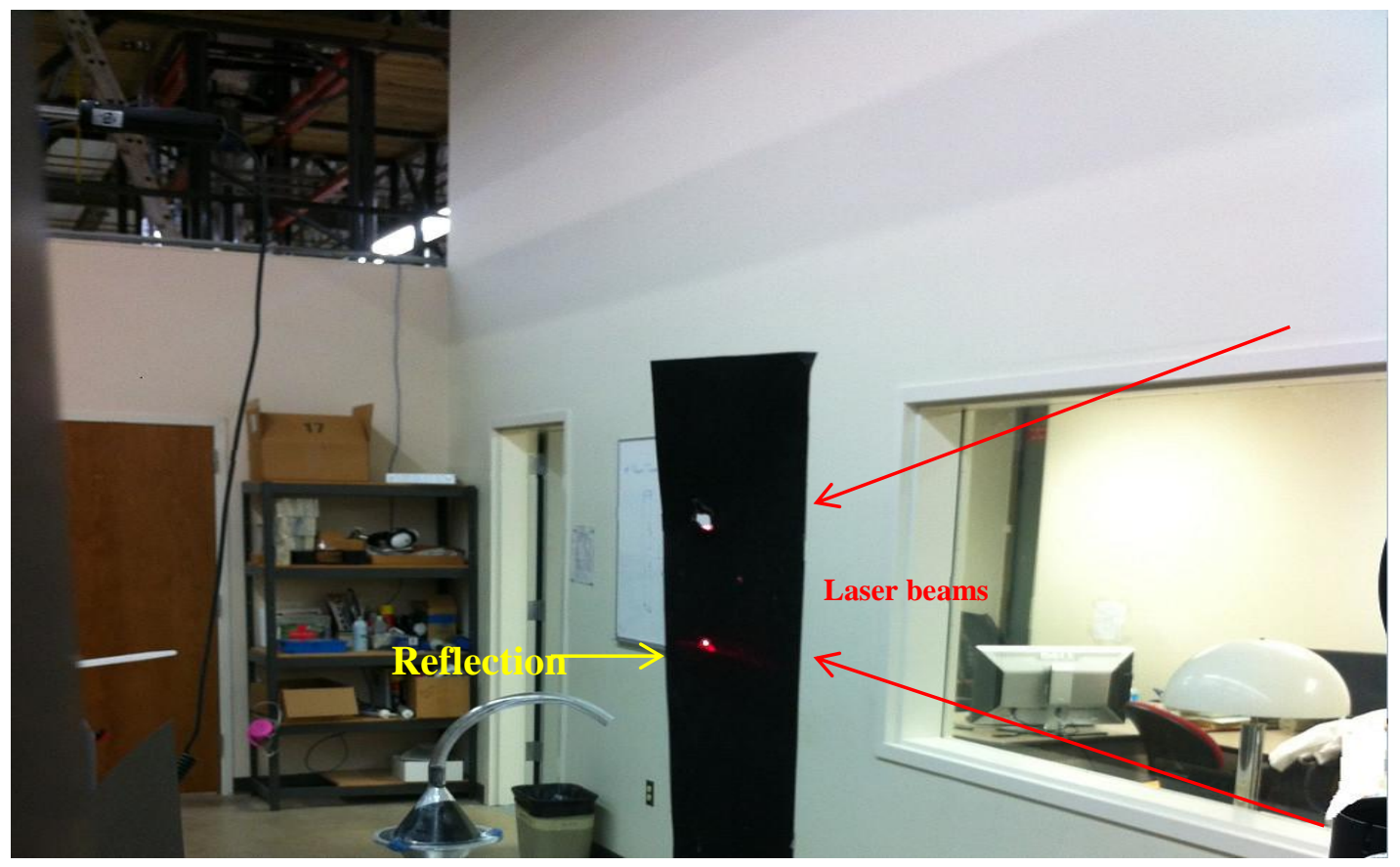

Fig.56 Examination of the optics alignment

\subsubsection{Scattered light interference}

The transparent acrylic material offers good optical transmission but it also creates some problems for the LDV measurements such as interference from the scattered light. Ideally, the photo detector in the LDV system should only receive the scattered light from the particles passing through the measuring volume. Other scattered lights resulted from the reflections within the experimental facility may create a significant amount of noises. Fig. 57 presents the black papers used to block the scattered light interference. It was found that the SNR was not satisfying without application of these black papers. Another benefit of using the black paper is that the 
horizontal beams used for measure the horizontal velocities were blocked too, which is necessary because the measurement is $1 \mathrm{D}$ only.

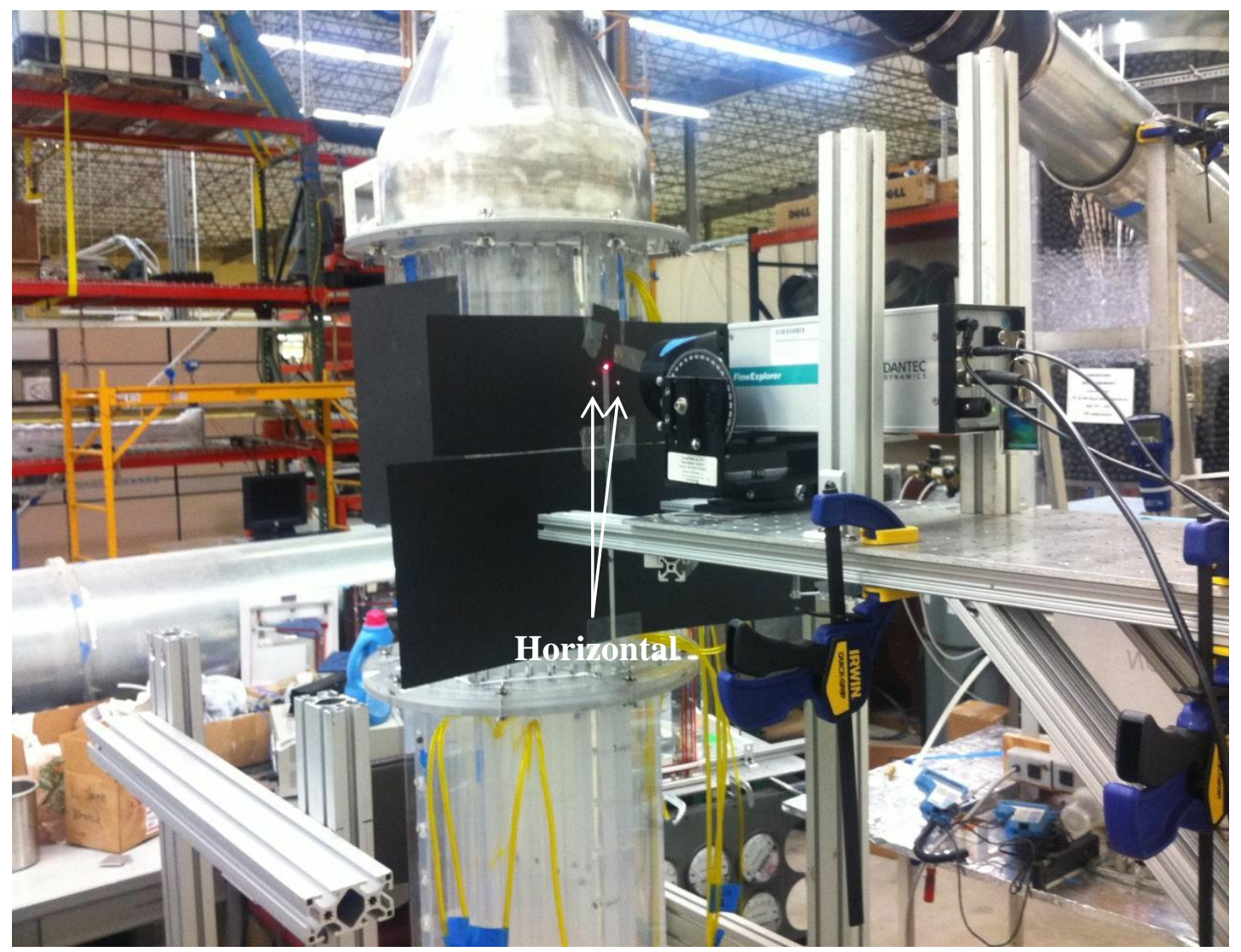

Fig.57 Reduction of the scattered light

\subsubsection{Wall effect}

The wall effect could be a major error of the LDV measurement as the particles collide with walls continuously. To reduce this error, the measuring volume was placed at the center of the gap. Furthermore, the starting position in the Y direction is $20 \mathrm{~mm}$ away from the gap divider or the wall instead of $10 \mathrm{~mm}$ as planned. It was because 
significant noises were observed which increased the uncertainties of the results. It can be observed from Fig. 58 that the noises at the near wall position $(\mathrm{y}=20 \mathrm{~mm})$ are more than that at $\mathrm{y}=110 \mathrm{~mm}$. A sample histogram obtained at $\mathrm{y}=60 \mathrm{~mm}$ at position $\mathrm{P} 1$ at $\operatorname{Re}_{\mathrm{BG}}=9,990$ is presented in Fig. 59.
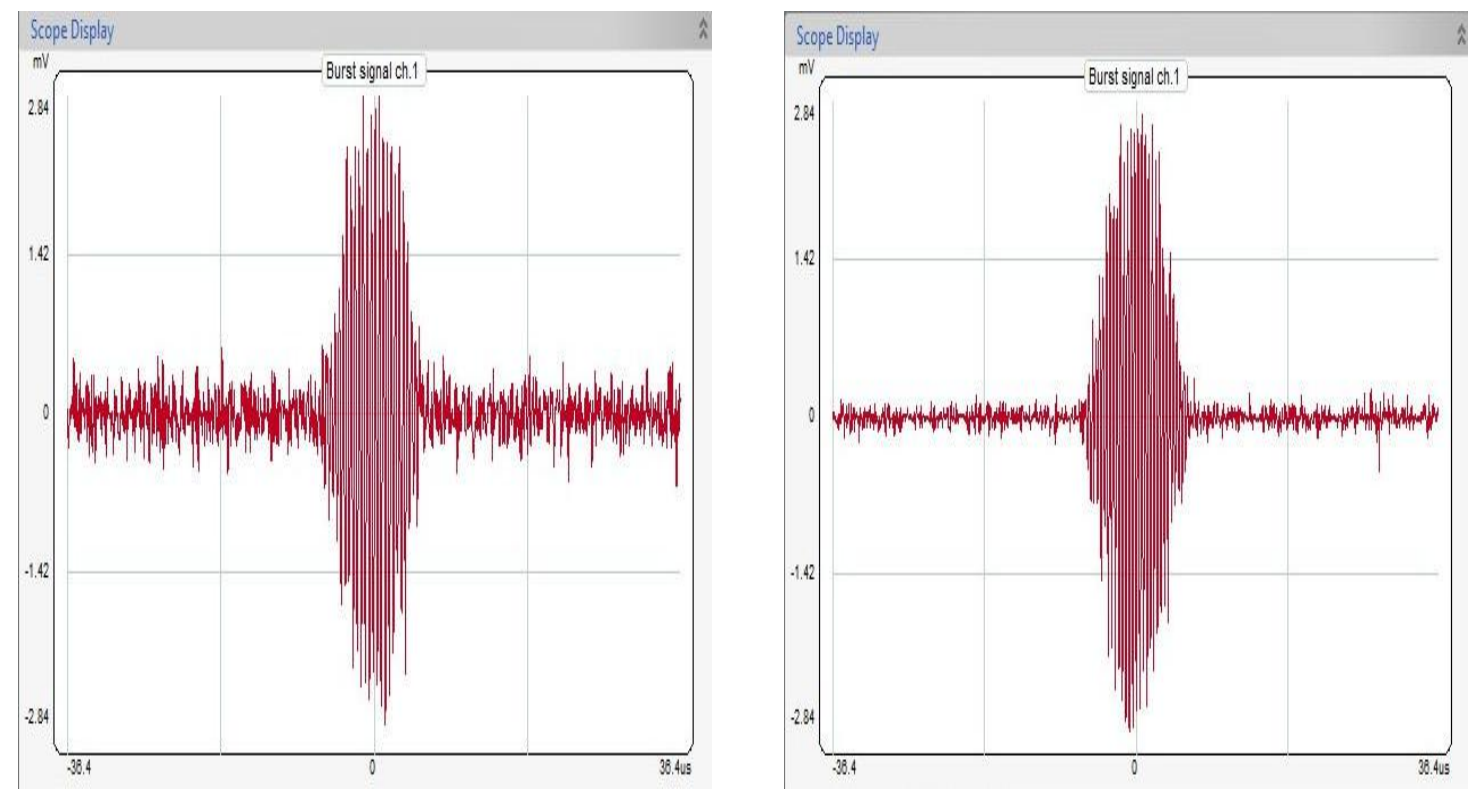

Fig.58 Signals obtained at $\mathrm{y}=20 \mathrm{~mm}$ (left) and $\mathrm{y}=110 \mathrm{~mm}$ (right) at $\mathrm{P} 2$ position 


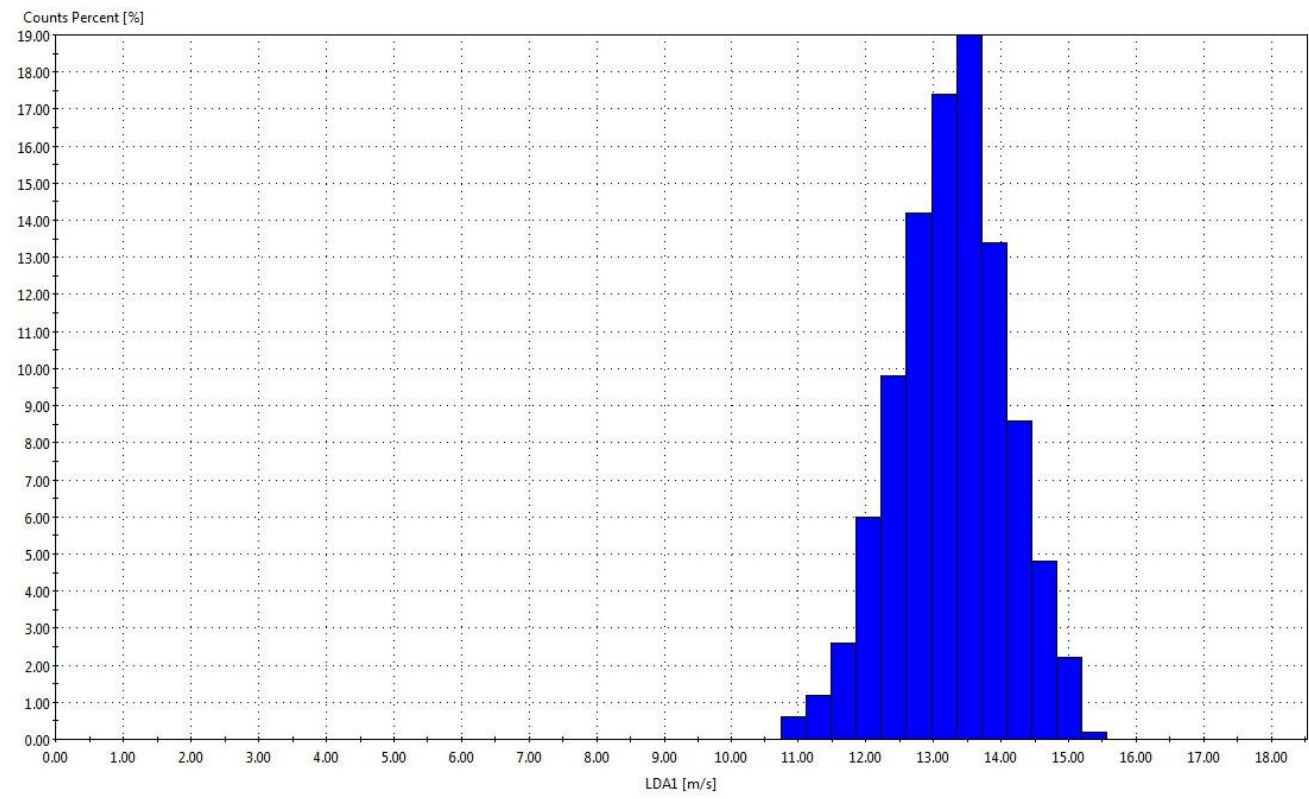

Fig.59 Histogram obtained at $\mathrm{y}=110 \mathrm{~mm}$ at $\mathrm{P} 1$ position with $\operatorname{Re}_{\mathrm{BG}}=9,990$

\section{UNCERTAINTY ANALYSIS}

To evaluate the bypass flow fraction of the TAMU experiment facility, volumetric flow rate $\mathrm{Q}$ of the inlet and three outlets needs to be measured accurately. However, due to some experimental limitations, such as the pressure drop within the inlet pipe is too low to apply an orifice flow meter, Q is estimated by the central velocities of the pipes using certain correlations, which addresses the importance of the accurate results from the velocity meters. Thus, a well calibrated Venturi flow meter was introduced and results from which will be treated as the reference.

The experimental loop consisted of blower, voltage transformer, Venturi flow meter, Manometer to measure the pressure drop and velocity meter and so on. The velocity probe was placed at the center of the 3 ' pipe. The flow rate was altered by 
changing the voltage inputted into the blower. The calibration results are demonstrated in Fig. 61.

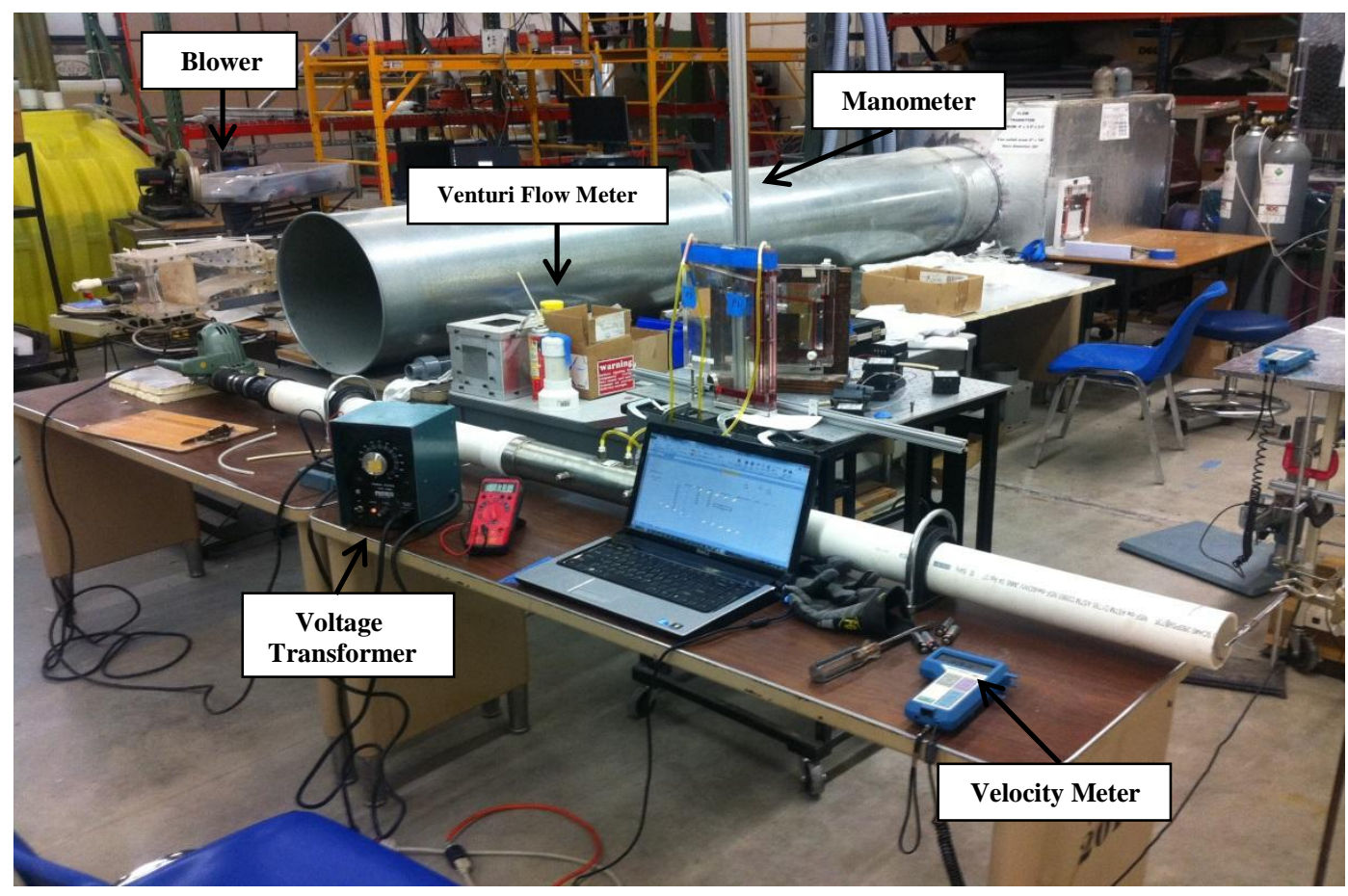

Fig. 60 The calibration Apparatus 


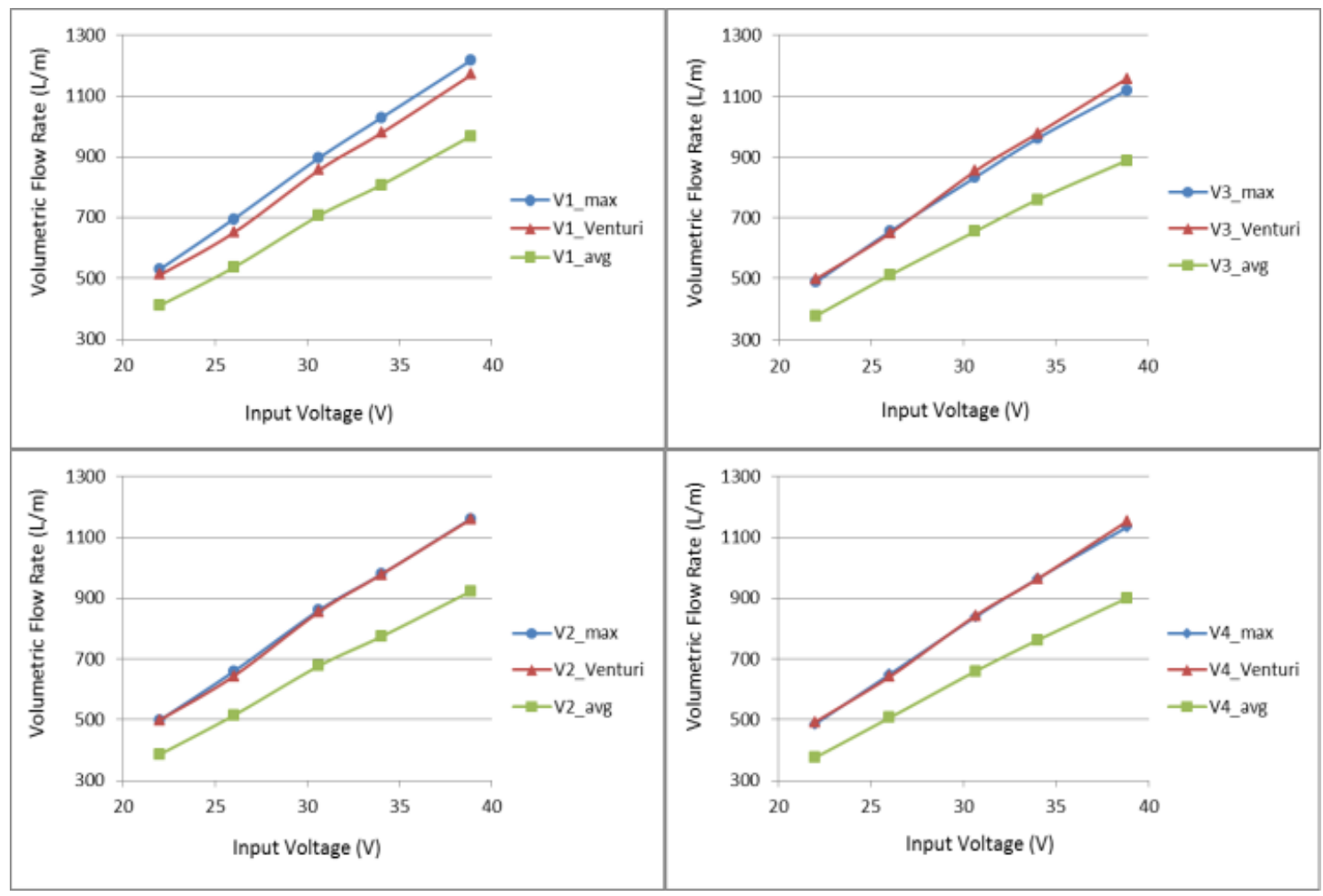

Fig.61 Calibrations of the velocity meters

The V_max the center velocity read from the meters while the V_avg is the calculated velocity using empirical correlations. It was found that the velocity meter 1 had the largest errors. Since the inlet mass flow rates were calculated using the center velocities in the inlet pipe, there might be errors involved because the center velocity and the average velocity used for calculating the flow rate are different. Fig. 62 presents the velocity distribution in the inlet pipe. ReIP represents the Reynolds number based on the inlet pipe. Only half of the velocity distribution is shown. The weighted average can be 
obtained using the fitting curves. The comparison between the center velocities and the weight ones are summarized in Table. 7. It is seen that the error is relatively small.

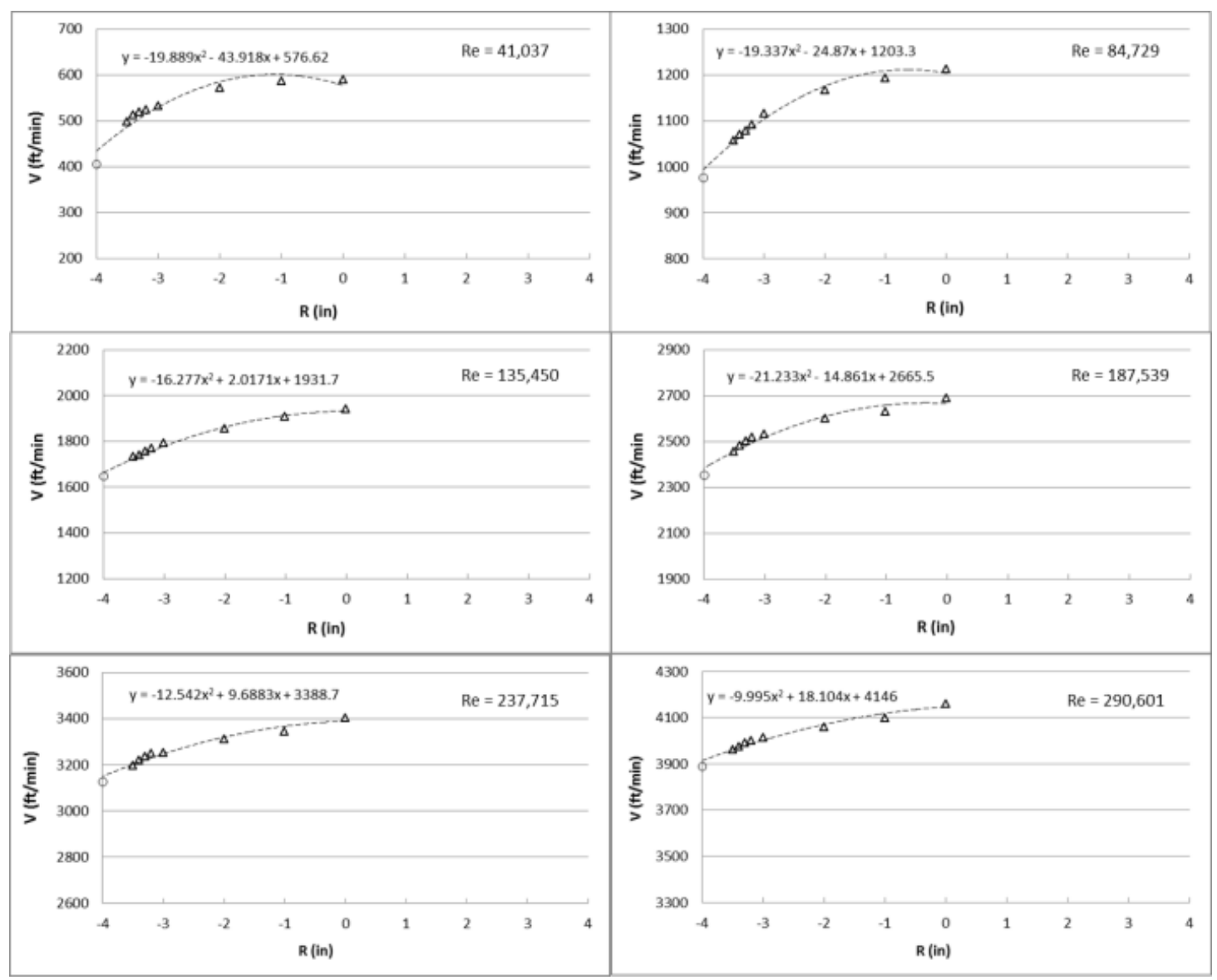

Fig.62 Velocity profiles in the inlet pipe 
Table.7 Comparisons between the weighted average velocities and the maximum velocities

\begin{tabular}{cccc}
\hline $\operatorname{Re}_{\mathrm{IP}}$ & Weighted average & Maximum & Difference (\%) \\
\hline 41,037 & 558 & 587 & 5.0 \\
84,729 & 1150 & 1213 & 5.2 \\
135,450 & 1841 & 1939 & 5.0 \\
187,539 & 2592 & 2684 & 3.4 \\
237,715 & 3302 & 3403 & 3.0 \\
290,601 & 4056 & 4160 & 2.5 \\
\hline
\end{tabular}

\section{CONCLUSION}

Air test of the coolant distribution in a two-block VHTR model built at Texas A\&M University was performed. The pressure drops in the facility are not significant which is comparable to the CFD simulation results. The bypass flow fraction ranges from $17.9 \%$ to $21 \%$. LDV technique was employed to investigate the velocity information within the bypass gap. It was found that the velocity is higher in the center of the facility. Uncertainty analysis was performed to quantify the errors from the velocity meters and the approximation in calculating the total mass flow rate. 